\title{
Lanthanide Molecules for Spin-based Quantum
}

\section{Technologies}

Guillem Aromí ${ }^{\mathrm{a}, \mathrm{b}}$ and Olivier Roubeau ${ }^{\mathrm{c}}$

${ }^{a}$ Departament de Química Inorgànica i Orgànica, Secció de Química Inorgànica, Universitat de Barcelona, Diagonal 645, 08028 Barcelona, Spain

${ }^{b}$ Institute of Nanoscience and Nanotechnology of the University of Barcelona, Barcelona, Spain

'Instituto de Ciencia de Materiales de Aragón (ICMA), CSIC, and Universidad de Zaragoza, Plaza San Francisco s/n, 50009, Zaragoza, Spain

\section{Abstract}

Since the molecular electronic spin emerged as one of the promising technologies to implement the quantum processing of information, coordination complexes of lanthanides and actinides have become protagonists as suitable molecular realizations of qubits and qugates. In this chapter we revise the most recent developments on the production of rare earth (RE) based qubits, qugates and most recently qudits, which also make use of the nuclear spin degrees of freedom to encode and process quantum information. Essential concepts such as the spin relaxation and the phase memory times in these systems are described, as well as the development of multiqubit molecular systems for the realization of basic quantum gate operations. The most advanced achievements consisting on the realization of the Grover algorithm or the demonstration of a quantum error correction protocol, constitute the culmination of this chapter, which concludes with the challenges yet lying ahead for implementing the molecular spin as an essential component of quantum computing. 


\section{Key Words}

Quantum Computing, Lanthanide Coordination Complexes, Slow Relaxation of the Magnetization, Quantum Coherence, Quantum Bit, Quantum Dit, Quantum Gate, Pulsed EPR, Magnetic Materials

\section{Chapter Outline}

1. Introduction

2. Spin relaxation dynamics of lanthanide and actinide single-molecule magnets (SMMs)

2.1 Slow relaxation of the magnetization and spin-lattice relaxation

2.2 Synthetic design of lanthanide SMMs and relevance as qubits.

2.2.1 Tuning anisotropy to control spin dynamics

2.2.2 Systems with high coordination number

2.2.3 Systems with high rotational symmetry ligands

2.2.4 Polyoxometallates (POMs)

2.3 Actinide SMMs

3. Quantum coherence of lanthanide and actinide spin-qubits

3.1 Causes of decoherence for spin qubits

3.2 Experimental estimation of quantum coherence of lanthanide and actinide qubits

3.2.1. Lanthanide ions doped in inorganic lattices as qubits

3.2.2 Lanthanide POMs as qubits

3.2.3 Lanthanide peptides

3.2.4 Organometallic actinide qubits

4. Multi-qubit quantum gates made of lanthanides

4.1 A dinuclear $\mathrm{Tb}(\mathrm{III})$ complex as prototype of a two-qubit quantum gate 
4.2 Heterometallic lanthanide complexes as quantum gates

4.3 Codification of several qubits within one lanthanide center

5. Realization of quantum algorithms with lanthanide qudits

5.1 Lanthanide electronic spins as qudits

5.2 Qudits encoded into the lanthanide nuclear spin

5.3 Realization of Grover algorithm with the Tb nuclear spin as qudit

5.4 An error-correction protocol using the nuclear spin of $\mathrm{Yb}$ as qudit

6. Conclusions

7. Acknowledgements

8. List of symbols and acronyms

9. References 


\section{Introduction}

Quantum technologies are one of the most relevant scientific developments to shape the (near) future. Indeed, they will be key to expand and/or have a huge impact on many branches of science and technology. Some examples are the understanding of quantum systems including the design of pharmaceutical therapies through quantum simulation [1], the area of data protection and secure communications thanks to quantum cryptography [2], or the processing of information through quantum computing [3], The central concept of quantum computing is that the information can be encoded and manipulated on quantum bits (or qubits), which may consist of any physical system able to generate quantum superpositions of two basis states (see Fig. 1). This implies a natural computational gain, since the size of the computer register required for an $\mathrm{N}$ component quantum problem grows only linearly with $N$, instead of the exponential growth occurring when using classical bits [3]. In addition, the manipulation of quantum information allows the exploitation of resources inherent to quantum mechanics, not available for classic computation. These are for example quantum superposition and quantum entanglement, which enable the development and use of algorithms that take advantage of quantum parallelism, thus providing avenues to solve information problems currently intractable.

The other key hardware ingredients are universal quantum logic gates that involve wiring various qubits (directly or through quantum buses) in such a way that they realize elaborate quantum operations [4]. There are many proposed candidates to embody qubits in quantum gates [5], among which superconducting circuits [6], trapped ions [7] and spins in solids [8-10] or 
molecules [11-12]. Each has advantages and weaknesses, but there are two key challenges that are common to all of them. One is the extreme fragility and associated loss of the quantum information due to interactions of the qubits with their environment, which is called decoherence [13]. And the other, termed scalability, is the necessity to have a large number of robust and reliable qubit identical replicas available [4]. Molecular spins could arguably be in a very advantageous position to meet both requirements. Compared with superconducting circuits and trapped ions, which are two of the most advanced qubit realizations, spins in general are relatively insensitive to electric field fluctuations. Thus spin-qubits may have excellent coherence, if protected from what they are sensitive to, which are magnetic field fluctuations. This has been largely demonstrated with the very long coherence times observed for the spins of NV (Nitrogen-Vacancy) centres in diamond [14] or phosphorous atom impurities in silicon [15]. Spins in molecules are comparatively less robust in terms of coherence, but it has been shown that synthetic strategies may open the way to reach sufficiently enhanced quantum coherence [11]. On the other hand, the ability of chemistry to produce large amounts of identical molecular qubit replicas or to build multi-qubit molecules able to encode a specific gate operation [16-17], while also controlling their organization, offers unique solutions to the scalability challenge.

Both, organic radicals and paramagnetic coordination compounds of first-raw transition metal ions have been studied as potential qubits [11-12, 18-21]. The latter in particular have witnessed significant improvement of their quantum coherence, with record values of the phase-memory or dephasing time $T_{\mathrm{m}}$ (see Fig. 1) reaching tens and even hundreds of microseconds for specific and 
optimized conditions [22-23]. Rare-earth (RE) complexes have been considered later, but have offered additional options that could prove very useful for quantum computation [24]. These are the possibility to encode multiple qubits in one sole ion [25], and the increased size of the quantum basis states by coupling the electronic spin with the nuclear spin [26]. So far, relatively few studies of lanthanide and actinide spin qubits exist. Some recent contributions are however particularly relevant for the development of quantum technologies, such as the proof-of-concept realization of quantum algorithms [27-28]. This chapter is an attempt to review the most significant contributions made recently on the preparation, physical description and implementation of lanthanide (Ln) and actinide (Ac) containing molecules for the advancement of quantum computing. We start with a non-exhaustive qualitative view on the spin dynamics of RE molecules in general (section 2), with a more detailed focus on those for which quantum coherence has been evaluated (section 3). Then, the use of lanthanide-based molecules for the realization of multi-qubit quantum gates (section 4) and quantum algorithms (section 5) is described, followed by some conclusions and outlook on the future of RE molecular materials in the development of spin-based quantum technologies.

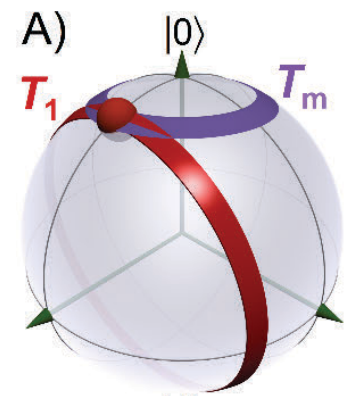

|1)

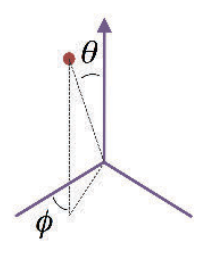

11)

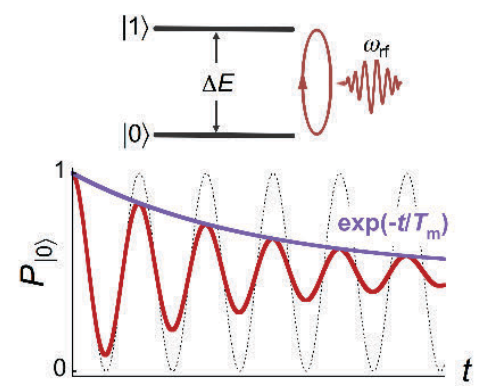

Rabi oscillations: $\Omega_{\mathrm{R}}$ 
Figure 1. A) Schematic representation of a qubit state. Each point on the surface of the Bloch sphere describes a quantum superposition of states 0$\rangle$ and $|1\rangle$ of the form $|\varphi\rangle=\cos (\theta / 2)|0\rangle+\exp (i \phi) \sin (\theta / 2)|1\rangle$. In a coherent manipulation of the qubit, both the amplitude $\theta$ and the phase $\phi$ must be controlled. The relaxation and decoherence or dephasing, governed by timescales $T_{1}$ and $T_{\mathrm{m}}$, respectively, are indicated in red and purple and correspond to randomization of $\theta$ and $\phi$. B) Schematic representation of coherent oscillations between states $|0\rangle$ and $|1\rangle$ of a qubit. These can be induced via the application of an external radio frequency (rf) pulse resonant with the energy gap separating the qubit basis states. The amplitude $\theta$ of the quantum superposition changes periodically at the Rabi frequency $\Omega_{R}$. Phase kicks cause the damping of these oscillations and thus the loss of coherence, as measured through $T_{\mathrm{m}}$. Reproduced from ref. [24] with permission.

\section{Spin relaxation dynamics of lanthanide and actinide single-molecule magnets (SMMs)}

In order to embody a spin qubit, any specific paramagnetic molecule requires that its electronic scheme provides a suitable basis for the qubit states, i.e. that under certain conditions it can be pictured as a two-level quantum system. This is obviously the case of a pure $S=1 / 2$ spin, but the peculiar magnetism of lanthanide ions also makes them, in most cases, good qubit candidates. On the one hand, the ground state $J$ multiplet is in general sufficient to accurately describe their magnetic properties, given the large energy differences between levels of different total angular momentum $J=L+S$ that result of the strong 
coupling between the orbital $L$ and spin $S$ angular momenta. Then, the splitting of the $2 J+1$ levels of this $J$ ground multiplet by interaction with the surrounding crystal field gives rise to a collection of $\pm m_{\lrcorner}$electronic levels. Of these, the lowest lying $\pm m_{\lrcorner}$doublet is generally well separated from all excited levels, therefore picturing most lanthanide ions as effective spins, $S_{\text {eff }}=1 / 2$ (Fig. 1).

The spin Hamiltonian of the ground multiplet can be written, in terms of Steven's formalism, as in Eq. 1.

$\widehat{H}=\sum_{n, m} B_{n}^{m} \hat{O}_{n}^{m}-g_{J} \mu_{B}\left(H_{x} J_{x}+H_{y} J_{y}+H_{z} J_{z}\right)$

In Eq. $1, B_{n}^{m}$ are anisotropy constants (with $m \leq n$ and $m$ even), $\hat{O}_{n}^{m}$ are effective spin operators containing powers of $J_{x}, J_{y}$ and $J_{z}$ (the latter being the three components of the total angular momentum $J$ ), and $g_{J}$ is the Ln ion gyromagnetic ratio. The anisotropy terms that are relevant depend on the local symmetry at the Ln ion, while their sign and magnitude are governed by the Ln nature as well as its local environment (symmetry and nature). Therefore, if most $\mathrm{Ln}$ and $\mathrm{Ac}$ ions are potentially good qubit candidates, their suitability will still strongly depend on their immediate local environment, their orientation with respect to an applied field and the overall environment within the solid lattice. It is thus difficult to predict if the spin relaxation time and specially the quantum phase coherence of the RE qubit candidates will be adequate. Surprisingly, there has been only very few studies oriented to elucidate precisely the suitability of RE ions as qubits. Since this is usually conducted through pulsedEPR techniques, this shortcoming may be in part due to broadening of EPR lines by e.g. zero-field splitting effects, the necessity of using uncommon EPR 
bands, or simply, the absence of any detectable spin echo in fast relaxing systems.

In fact, the spin relaxation behaviour of REs was originally intensively studied as dopants of various crystalline solids, which provided much of the general basis for the theory of spin-lattice relaxation [29-30]. Subsequently, the fast dynamics generally observed for RE ions attracted interest for their potential as relaxing agent of other spins. This has been specially the case with $\mathrm{Gd}(\mathrm{III})$, becoming very important as contrast agent for this reason [31]. The slow dynamics of lanthanide spin-lattice relaxation has instead attracted a great deal of interest only recently, because of the surge of single-molecule magnets (SMMs) and in particular those based on a single metal ion. Indeed, the observation of SMM properties in $\left[\mathrm{NBu}_{4}\right]\left[\mathrm{TbPc}_{2}\right]$ showed that monometallic $4 \mathrm{f}$ compounds could serve to retain magnetic memory at the molecular level [32]. Since then, huge amounts of literature have been gathered on $4 \mathrm{f}-\mathrm{SMMs}$, in particular based on Dy(III) systems, in which the spin relaxation dynamics of monometallic lanthanide complexes is studied through ac susceptibility and rationalized in terms of anisotropy (and energy barrier for the magnetic relaxation) [33-37].

The potential of RE ions as spin qubits requires reasonably long quantum phase coherence times $T_{\mathrm{m}}$, but $T_{\mathrm{m}}$ can easily be limited by the spin-lattice relaxation $T_{1}$. Since not all RE SMMs can be good spin qubits, the chemical design of Ln SMMs and the rationalization of long spin-lattice relaxation are highly relevant in general for the use of REs in quantum technologies. This section therefore summarizes aspects of the intense research done on Ln and Ac monometallic SMMs relevant for the subject of this review, together with a 
more detailed description of the few systems for which time-domain EPR studies have been performed.
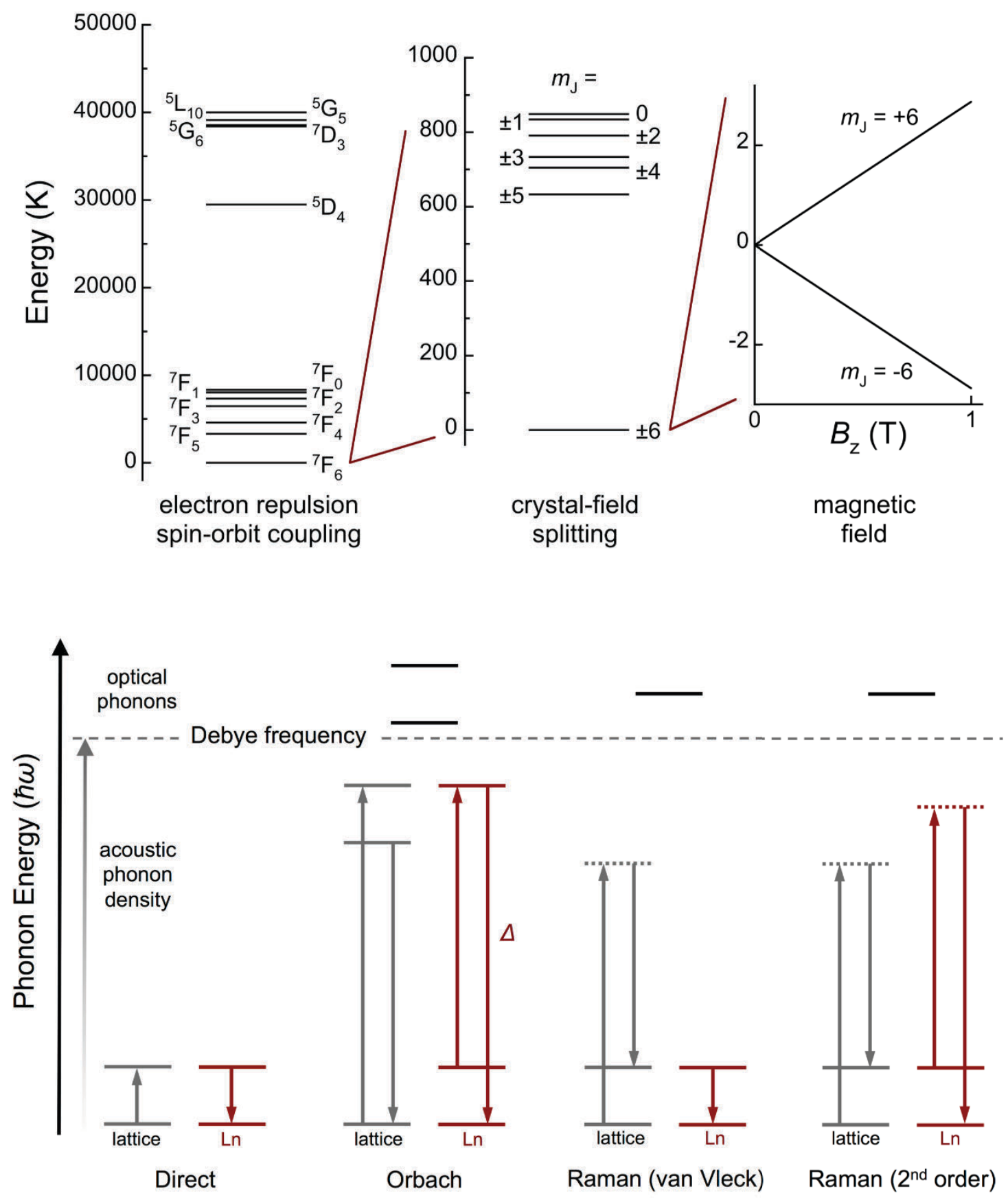

Figure 2. (Top) Typical energy level scheme for a lanthanide ion describing the effect of electronic and magnetic field (Zeeman) interactions and their typical magnitudes. The example corresponds to a $\mathrm{Tb}(\mathrm{III})$ ion. (Bottom) Schematic representation of the different processes involved in spin-lattice relaxation for a 
(Kramers) doublet of a lanthanide split by Zeeman interaction (dark red). Both first and second order Raman processes are shown. The Orbach process is depicted as two consecutive direct transitions, rather than as resonance Raman process as sometimes done. Higher energy optical phonons are included to indicate these can also be involved in the Orbach and Raman mechanism.

\subsection{Slow relaxation of the magnetization and spin-lattice relaxation}

The electron spin relaxation has been described in terms of transitions between spin states involving the exchange of energy with the environment. The different processes contributing to the spin-lattice relaxation rate $1 / T_{1}$ were all proposed and defined in between the 1940's and the 1960's, and have been described in detail in various review reports [29, 38-39]. The term spin-lattice relaxation evokes the fact that this phenomenon was originally studied on crystalline solids, however the word "lattice" currently designates any environment or surroundings of the spin carrier. Reasonably, long $T_{1}$ values are a pre-requisite for a spin qubit as $T_{1}$ can pose a limitation of the quantum coherence time, especially at high temperatures. The following is a brief description of the different mechanisms of the spin-lattice relaxation.

i) Direct process. In this relaxation path, the electron spin transition energy exactly matches that of a phonon (Fig. 2, bottom). At low temperatures, i.e. well below the Debye temperature, the phonon density of states results in a linear increase of the direct process relaxation rate $T_{\text {dir }}^{-1}$ with temperature. Generally,

$T_{\text {dir }}^{-1}$ is expressed as $A_{\text {dir }} H^{n} T, n$ being 4 for Kramers ions. Because of the relatively low number of phonons matching specific transitions, the direct 
process is often not particularly efficient in lanthanides, but it can be dominant at high fields.

ii) Raman and Orbach processes. Contrary to the direct process, the Raman and Orbach processes engage two-phonons, some of higher energy that that of the spin transition (Fig. 2, bottom). This makes these processes more efficient, especially at higher temperatures. In both cases, the energy exchanged with the lattice is the energy difference between the absorbed and emitted phonons for a given excited state. In the Orbach process, the excited state is a real electronic state, while in the Raman process it is a virtual excited state of any energy lower than the Debye temperature. The temperature dependence of the relaxation rates are typically expressed as $T_{\text {Orbach }}^{-1}=\Delta^{-3} \exp \left(-\Delta / k_{B} T\right)$ and $T_{\text {Raman }}^{-1}=A_{\text {Raman }} T^{m} . \Delta$ is the energy gap involved in the transition between real electronic states, but it does not necessarily correspond to the transition from the ground state to the first excited state. In Ln systems, it is in fact common that one or more transition involving excited $m_{\mathrm{J}}$ states are active Orbach processes. The exponent $m$ can take values between 2 and 9 depending on the type of Ln ion (Kramers or non-Kramers) and other effects such as interactions or the presence of a phonon bottleneck [29, 40].

iii) Quantum tunneling. This process depends on the probability of tunneling through the energy barrier between degenerate $\pm m_{\mathrm{J}}$ states. This process is naturally temperature independent and is significant only at low temperatures, although thermally-activated tunneling processes between excited $\pm m_{\mathrm{J}}$ states can also be relevant. Because this mechanism requires the $\pm m_{J}$ states involved to be degenerate, it is often quenched under an applied $d c$ field. The field dependence of the rate corresponding for this process is $T_{Q T M}^{-1}=\frac{B_{1}}{1+B_{2} H^{2}}$. 
Experimentally, $T_{1}$ can be determined by any physical technique sensitive to the relative population of spin states in the appropriate time-scale. Pulsed-EPR appears ideal as it is a direct measure of the electron spin relaxation. Nevertheless, there are only very few reports on the determination of $T_{1}$ of $\mathrm{Ln}$ and Ac ions by EPR, possibly due to broadening of EPR lines or the absence of any detectable echo. The slow magnetic relaxation of SMMs is indeed related to long spin relaxation times, and the determination of the associated relaxation rate $\tau$ is now routinely done through ac susceptibility measurements, or even $d c$ isothermal measurements $v s$. time when relaxation times are sufficiently long. In principle, $\tau$ can be taken as an effective value of $T_{1}$, and the vast majority of available spin-lattice relaxation rates for REs arise thus from studies of RE monometallic SMMs, which motivates the focus of this section.

When available, the field and temperature dependence of $\tau$ (or $T_{1}$ ) can be used to evaluate the different relaxation paths at work, which is typically done by fitting the variable temperature data to the expression in Eq. 2 .

$$
\tau^{-1}=\frac{B_{1}}{1+B_{2} H^{2}}+A_{d i r} H^{n} T+A_{\text {Raman }} T^{m}+\Delta^{-3} \exp \left(-\frac{\Delta}{k_{B} T}\right)
$$

This expression is amenable to some simplifications through sensible assumptions allowing to neglect one or more of its terms, while ab initio calculations and/or luminescence data providing energy level schemes can be very helpful (a typical example in Fig. 3). Nevertheless, it generally remains very challenging to extract with confidence the characteristic parameters of each process from such a multi-parameter fit. This is particularly true considering that in general the experimentally-determined $T_{1}$ is the sum of all possible relaxation 
pathways and that: i) there can be additional local processes such as spectral diffusion, nuclear spin relaxation or molecular motions mixing spin and orbital angular momentum [39], ii) various processes can be competing in the same temperature/field range; iii) phonon bottleneck effects can strongly affect spin relaxation in concentrated solids $[29,40]$.

In practice, the most commonly determined parameters for SMMs is rather an effective energy barrier to relaxation, $U_{\text {eff, }}$ determined by applying an Arrheniustype expression $\tau=\tau_{0} \exp \left(U_{e f f} / k_{B} T\right)$ to the temperature dependence of $\tau$. Although this is done typically from higher temperature data, this would imply that the dominant relaxation path is of Orbach-type. In reality, this remains a crude approximation, since some studies involving detailed relaxation data indicate that Raman processes are in some compounds controlling the spin dynamics [41-42]. In particular, for systems with high $U_{\text {eff }}$ values, mostly involving $\mathrm{Dy}(\mathrm{III})$ and $\mathrm{Tb}(\mathrm{III})$, a correlation between $\tau_{\text {Raman }}$ and the so-called blocking temperature of the SMM (limiting temperature of detection of the hysteresis) could be derived [43].
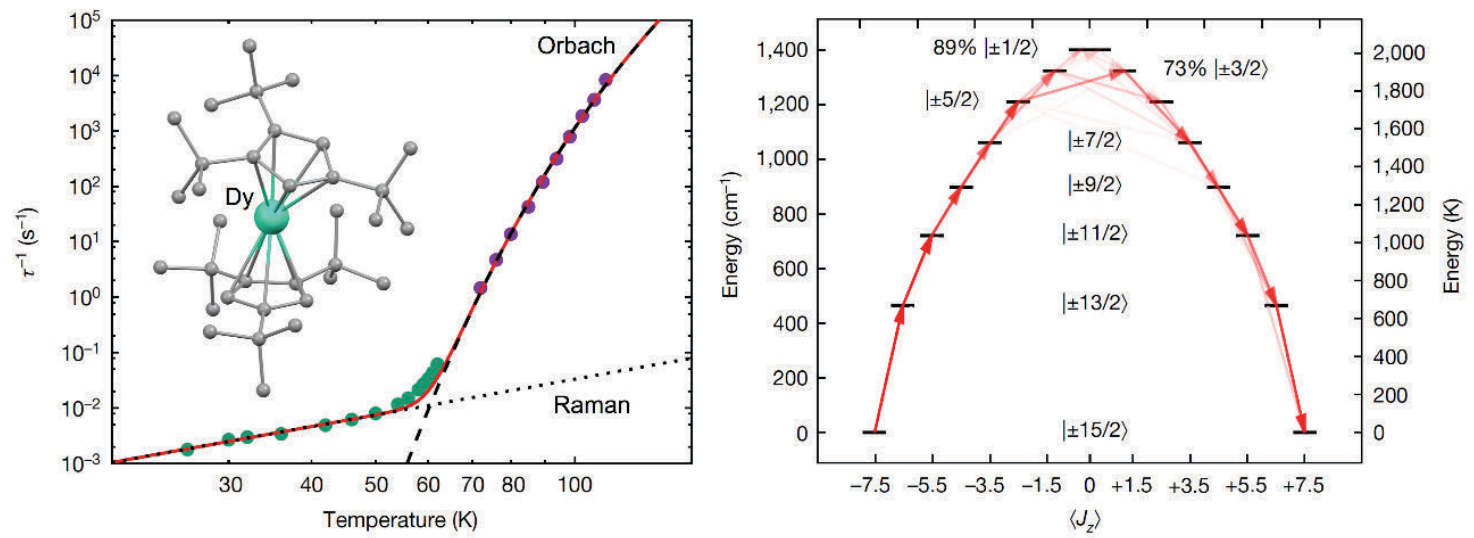

Figure 3. Typical characterization and analysis of the spin-lattice relaxation in Ln SMMs. (Left) Temperature dependence of the relaxation rate $\tau^{-1}$ of $\left[\mathrm{Dy}\left(\mathrm{Cp}^{\mathrm{ttt}}\right)_{2}\right]\left[\mathrm{B}\left(\mathrm{C}_{6} \mathrm{~F}_{5}\right)_{4}\right][44]$, as extracted from ac susceptibility (purple dots) and 
$d c$ magnetization decay (green dots). The data was satisfactorily reproduced (full red line) with the sum of Orbach (dashed line) and Raman (dotted line) relaxation processes. The structure of the cation $\left[\mathrm{Dy}\left(\mathrm{Cp}^{\mathrm{ttt}}\right)_{2}\right]^{+}$is shown as inset. (Right) Electronic states and energy barrier to relaxation for $\left[\mathrm{Dy}\left(\mathrm{Cp}{ }^{\mathrm{ttt}}\right)_{2}\right]\left[\mathrm{B}\left(\mathrm{C}_{6} \mathrm{~F}_{5}\right)_{4}\right]$ from ab initio calculations (CASSCF-SO). Arrows represent the calculated Orbach relaxation pathway(s) at $150 \mathrm{~K}$, with the opacity of the arrows proportional to the single-phonon transition probability. Reproduced from ref. [44] with permission. 
Table 1. Characteristics of the experimental spin-lattice relaxation time $T_{1}$ for a selection of lanthanide monometallic SingleMolecule-Magnets, together with $T_{1}$ and phase-memory times, $T_{\mathrm{m}}$, for systems studied through pulsed-EPR techniques.

\begin{tabular}{|c|c|c|c|c|c|c|c|c|}
\hline Complex & local symmetry & $U_{\text {eff }} / k_{B}{ }^{*}$ & $\max \tau @ \mathrm{~T}^{\star *}$ & $\max T_{1} @ \mathrm{~T}$ & $\max T_{m} @ \mathrm{~T}$ & $\Omega_{\mathrm{M}}$ & conditions & ref \\
\hline$\left(\mathrm{NBu}_{4}\right)\left[\mathrm{TbPc}_{2}\right]$ & $\approx D_{4 \mathrm{~d}}$ & 330 & & & & & & [32] \\
\hline$\left(\mathrm{NBu}_{4}\right)\left[\mathrm{DyPc}_{2}\right]$ & $\approx D_{4 \mathrm{~d}}$ & 40 & & & & & & [32] \\
\hline$\left[\mathrm{TbPc}_{2}\right]$ & $\approx D_{4 \mathrm{~d}}$ & 590 & & & & & & [45] \\
\hline [TbPcPc'] & $\approx D_{4 \mathrm{~d}}$ & 938 & $45 \mathrm{~ms} @ 41 \mathrm{~K}$ & & & & & [46] \\
\hline$\left(\mathrm{NBu}_{4}\right)\left[\mathrm{Tb}\left(\mathrm{Pc}(\mathrm{OPhtBu})_{8}\right)_{2}\right]$ & $\approx D_{4 \mathrm{~d}}$ & 701 & $22 \mathrm{~ms} @ 34 \mathrm{~K}$ & & & & & [46] \\
\hline$\left[\left(\mathrm{Cp}^{*}\right) \operatorname{Er}(\mathrm{COT})\right]$ & $\approx C_{\infty \mathrm{V}}$ & 323 & $40 \mathrm{~ms} @ 16 \mathrm{~K}$ & & & & & {$[47]$} \\
\hline$[\mathrm{K}(18 \mathrm{C} 6)]\left[\mathrm{Dy}(\mathrm{COT})_{2}\right]$ & $\approx D_{8 \mathrm{~d}}$ & 286 & $0.2 \mathrm{~ms} @ 4 \mathrm{~K}$ & & & & & [48] \\
\hline$[\mathrm{K}(18 \mathrm{C} 6)]\left[\mathrm{Er}(\mathrm{COT})_{2}\right]$ & $\approx D_{8 \mathrm{~h}}$ & 11 & $>12 \mathrm{~s}<12 \mathrm{~K}$ & & & & & [48] \\
\hline$\left[\mathrm{Dy}\left(\mathrm{Cp} \mathrm{ptt}_{2}\right]^{+}\right.$ & & 1760 & $\begin{array}{l}400 \mathrm{~s} @ 25 \mathrm{~K} \\
0.1 \mathrm{~ms} @ 112 \mathrm{~K}\end{array}$ & & & & & [44] \\
\hline$\left[\mathrm{Dy}\left(\mathrm{BIPM}^{\mathrm{IMS}}\right)_{2}\right]^{-}$ & pseudo- $D_{5 \mathrm{~h}}$ & 813 & $\begin{array}{l}>1 \mathrm{~s} @ 22 \mathrm{~K} \\
0.2 \mathrm{~ms} @ 42 \mathrm{~K}\end{array}$ & & & & & [49] \\
\hline$\left[\mathrm{Dy}\left({ }^{\mathrm{t}} \mathrm{BuO}\right)_{2}(\mathrm{py})_{5}\right]^{+}$ & pseudo- $D_{5 \mathrm{~h}}$ & 1815 & $>1 \mathrm{~s} @ 38 \mathrm{~K}$ & & & & & {$[50]$} \\
\hline
\end{tabular}




\begin{tabular}{|c|c|c|c|c|c|c|c|c|}
\hline & & & 75 us @100K & & & & & \\
\hline$\left[\left(\mathrm{L}_{\text {Klaui }}\right) \mathrm{Gd}(\right.$ porph $\left.)\right]$ & & & & $43 \mu \mathrm{s} @ 3 \mathrm{~K}$ & $3.6 \mu s @ 3 \mathrm{~K}$ & - & frozen solution & {$[51]$} \\
\hline $\mathrm{Gd}_{2} @ \mathrm{C}_{79} \mathrm{~N}$ & & & & $6 \mu \mathrm{s} @ 6 \mathrm{~K}$ & $5 \mu \mathrm{s} @ 6 \mathrm{~K}^{\mathrm{a}}$ & & frozen solution & {$[52]$} \\
\hline $\mathrm{Er@CaWO} 4$ & $\approx S_{4}$ & & & & $7 \mu \mathrm{s} @ 3.5 \mathrm{~K}$ & $10^{4} @ 1.5 \mathrm{~K}$ & single-crystal & [53] \\
\hline${ }^{167} \mathrm{Er} @ \mathrm{CaWO}_{4}$ & $\approx S_{4}$ & & & & $50 \mu \mathrm{s} @ 2.5 \mathrm{~K}$ & & single-crystal & {$[54]$} \\
\hline$\left[\mathrm{Er}\left(\mathrm{W}_{5} \mathrm{O}_{18}\right)_{2}\right]^{9-}$ & $\approx D_{4 \mathrm{~d}}$ & 56 & & & & & & {$[55]$} \\
\hline$\left[\mathrm{Gd}\left(\mathrm{W}_{5} \mathrm{O}_{18}\right)_{2}\right]^{\mathrm{ge}}$ & $\approx D_{4 \mathrm{~d}}$ & 2.2 & $67 \mathrm{~ms} @ 200 \mathrm{mK}$ & & & & powder & {$[56]$} \\
\hline$\left[\mathrm{Ho}\left(\mathrm{W}_{5} \mathrm{O}_{18}\right)_{2}\right]^{9-}$ & $\approx D_{4 \mathrm{~d}}$ & & & $20 \mu \mathrm{s} @ 5 \mathrm{~K}$ & $8.4 \mu \mathrm{s} @ 5 \mathrm{~K}$ & $\approx 820$ & single-crystal & {$[57]$} \\
\hline$\left[\mathrm{DyP}_{5} \mathrm{~W}_{30} \mathrm{O}_{110}\right]^{12-}$ & $\approx C_{5}$ & 24 & & & & & & {$[58]$} \\
\hline$\left[\mathrm{GdP}_{5} \mathrm{~W}_{30} \mathrm{O}_{110}\right]^{1 \mathrm{Z}^{-}}$ & $\approx C_{5}$ & & $10 \mu \mathrm{s} @ 200 \mathrm{mK}$ & $10 \mu \mathrm{s} @ 6 \mathrm{~K}$ & $0.6 \mu s @ 6 \mathrm{~K}$ & $\geq 50$ & powder & {$[56]$} \\
\hline [Yb(trensal)] & $C_{3 v}$ & 55 & $\begin{array}{l}180 \mathrm{~ms} @ 0.6 \mathrm{~K} \\
1 \mathrm{~ms} @ 4 \mathrm{~K}\end{array}$ & $8 \mathrm{~ms} @ 5 \mathrm{~K}$ & $0.5 \mu \mathrm{s} @ \leq 8 \mathrm{~K}$ & 40 & frozen solution & $\begin{array}{l}{[42]} \\
{[59]}\end{array}$ \\
\hline
\end{tabular}

${ }^{a}$ dynamic decoupling

* Effective energy barrier as determined by applying an Arrhenius-like expression to the temperature dependence of the relaxation time determined through ac susceptibility. When more than one relaxation mode exists (i.e. there is more than one $\cup_{\text {eff }}$ ), the largest $U_{\text {eff }}$ is given.

${ }^{* *}$ relaxation time $\tau$ determined through ac susceptibility, and taken as equivalent to $T_{1}$ as first approximation. 


\subsection{Synthetic design of lanthanide SMMs and relevance as qubits.}

\subsubsection{Tuning anisotropy to control spin dynamics}

In Ln and Ac ions, crystal field effects define the spin relaxation behaviour as these are the main source of magnetic anisotropy, which is responsible of the splitting of the spin-orbit coupling levels (Fig. 2, top). Thus, the local geometry and/or symmetry and the identity of the Ln are paramount and can be tuned synthetically to favour certain ground $\pm m_{\lrcorner}$states and adjust their splitting. In SMM research, the goal is to have the highest possible $m_{\lrcorner}$value as the ground state and to minimize QTM effects to achieve bistability at the highest possible temperature. A qualitative electrostatic approximation was first delineated by considering the interaction of the electron density of a given $\operatorname{Ln}(\mathrm{III})$ free ion with different crystal fields [60-61]. Thus, the radial distribution of the $4 \mathrm{f}$ electron shell, especially of the levels with highest $\pm m_{J}$ values, is seen to vary along the Ln series from prolate axial elongation to oblate equatorial elongation (Fig. 4). This means that the desired energy level configuration with the highest $m_{J}$ lowest in energy is expected to be favoured by a strongly axial/equatorial ligand field respectively for oblate/prolate ions. Ab initio calculations confirmed this qualitative picture [62], also predicting larger energy barriers, $U_{\text {eff, }}$ for systems with stronger axiality. In particular, Ln ions with oblate electron density of their levels with largest $m_{\lrcorner}$values, such as $\mathrm{Dy}(\mathrm{III})$ or $\mathrm{Tb}(\mathrm{III})$, would present huge anisotropy and $U_{\text {eff }}$ when 2-coordinated, especially with negatively charged ligands, because the resulting linear environment with very short Ln-L bond lengths maximizes axiality [63-64]. In recent years, significant synthetic efforts embracing these considerations have allowed to successfully isolate Ln SMMs exhibiting magnetic bistability at increasingly higher temperatures [33-37]. It 
should however be pointed out that rationalization and control of magnetic anisotropy can be more complex than the simple picture given here [65]. Another important aspect of SMMs is that QTM relaxation may be suppressed by relatively weak $d c$ magnetic fields, of the order of the internal crystal field, able to bring the ground doublet out of resonance. This is comparatively less relevant for qubits, since these would be manipulated under a local $d c$ field in any case.

Low coordination numbers are extremely difficult to obtain synthetically for $f$ elements, due to their large orbital radii and electronic charges. Synthetic efforts have thus aimed more achievable and stable environments through diverse strategies, still able to favour strong axiality at the Ln ion, and thus to maximize magnetic anisotropy. In the following we briefly discuss relevant synthetic and structural aspects that could be useful for the design of Ln qubits with long $T_{1}$, detailing, when available, examples for which pulsed-EPR studies have been performed (Table 1). The reader is otherwise referred to recent reviews gathering most data of the literature on Ln and Ac SMMs [33-37].

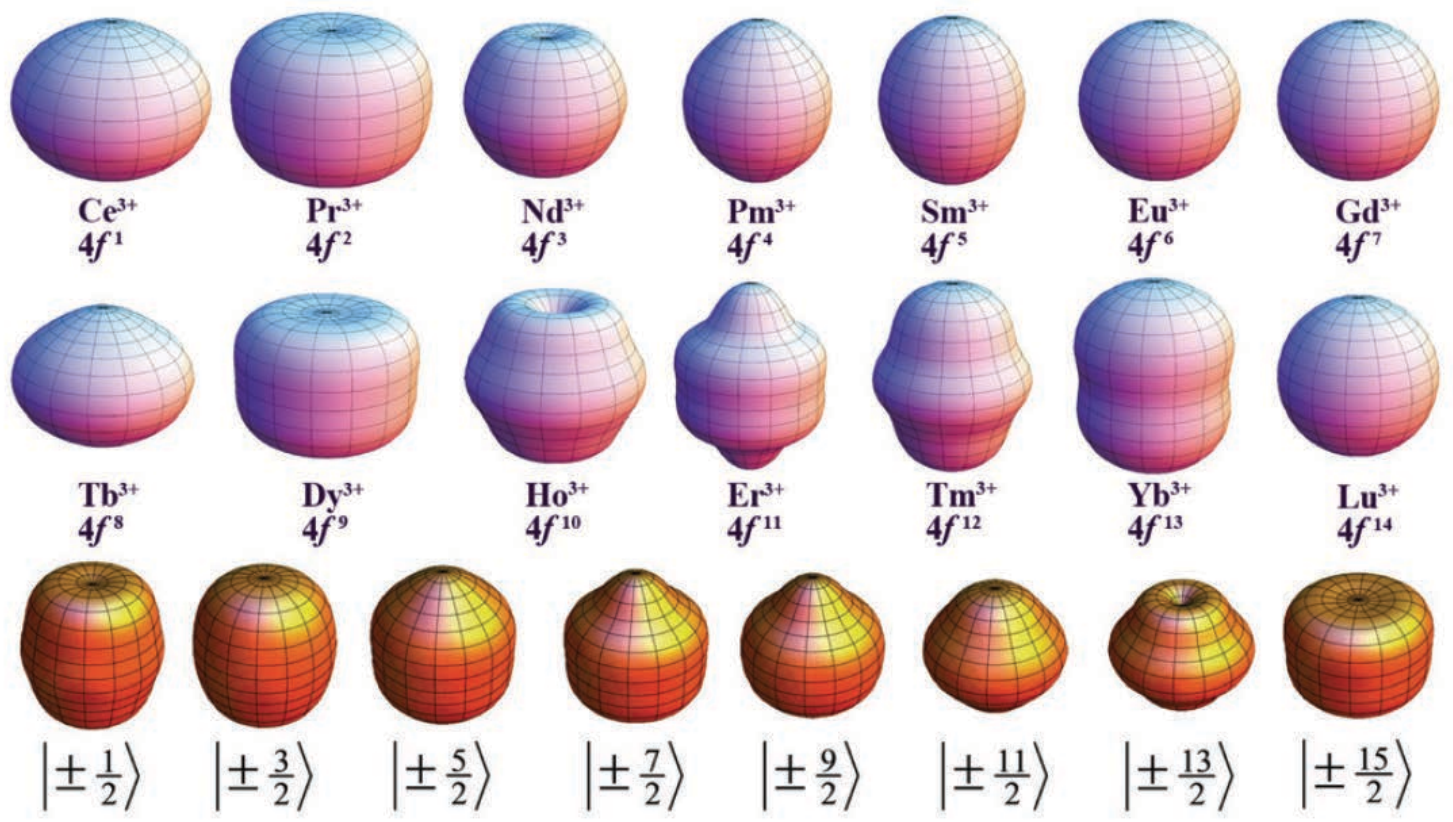


Figure 4. Angular dependence of the $4 \mathrm{f}$ charge-density. Top two rows: shown for the Ising state with $m_{J}= \pm J$ of all lanthanides. Bottom row: shown for each $m_{\lrcorner}$states of the Dy(III) multiplet from prolate $\left(m_{\lrcorner}= \pm 1 / 2\right)$ to oblate shape $\left(m_{\lrcorner}=\right.$ $\pm 15 / 2$ ). Reproduced from ref. [66] with permission.

\subsubsection{Systems with high coordination numbers}

Ln ions with high-coordination numbers and no symmetric environments can also exhibit interesting spin dynamics. As shown through the SMM literature, most Dy(III) complexes, independently of the coordination number, show some slow relaxation of their magnetization, at least under an applied $d c$ field and at sufficiently low temperatures. Another example of asymmetric environments with relevance for quantum information processing is found in the asymmetric dimetallic system used to design 2-qubit gates described in sections 4.1 and 4.2 [67-68]. The heterometallic analogues [ $\mathrm{LaEr}]$ and [CeY] indeed provide proper definitions of isolated qubits, even if no spin nor phase relaxation times were determined; the fact the [CeEr] complex does present quantum coherence $\left(T_{\mathrm{m}}\right.$ of the order of $0.4 \mu \mathrm{s}$ ) suggests sufficiently long spin-lattice relaxation times for the isolated qubits. Similarly, no particular design favouring axiality is used in the lanthanide binding tags used in biology, and still their quantum coherence is used to study protein structure. In fact, following a proposal to use Ln-peptides as platforms for quantum computing, $\mathrm{Nd}(\mathrm{III})$ and $\mathrm{Er}(\mathrm{III})$ peptides have been studied showing $T_{1}$ values of 8 and $360 \mu$ s, respectively [69].

One way to favour large anisotropy at Ln sites with relatively high coordination numbers is to have at least one short $L n-L$ bond ( $L=a n y$ donor atom). This is the case for poly-lanthanide alkoxide cages with $\mathrm{LnO}_{6}$ sites, in which a clear 
anisotropy axis is formed along a $\mathrm{O}_{\mathrm{ox}}-\mathrm{Ln}-\mathrm{O}_{\text {alk }}$ direction $\left(\mathrm{O}_{\mathrm{ox}}\right.$ and $\mathrm{O}_{\text {alk }}$ being central oxide and terminal alkoxide, respectively), $\mathrm{Ln}-\mathrm{O}_{\text {alk }}$ being much shorter than the other bond [70]. In Dy-doped $Y$ analogues, this actually gives rise to very large energy barriers above $800 \mathrm{~K}$, resulting from the reduced relaxation through the first excited stated together with the suppression of zero-field QTM between the $\pm 15 / 2 m_{J}$ ground states of the Dy(III) ion.

A more efficient means to favour a pseudo-linear local environment is to have two negatively charged donors disposed in trans along a principal axis, with weaker donors completing the coordination sphere in an equatorial plane. An elegant method to achieve this is using chelating polydentate ligands with both types of donors, a good example being $\left[\mathrm{K}(18 \mathrm{C} 6)(\mathrm{THF})_{2}\right]\left[\mathrm{Dy}\left(\mathrm{BIPM}^{\mathrm{TMS}}\right)_{2}\right]$ $\left(\mathrm{BIPM}^{\mathrm{TMS}}=\left(\mathrm{C}\left(\mathrm{PPh}_{2} \mathrm{NSiMe}_{3}\right)_{2}\right)^{2-}\right)$ with two capping tridentate carbene ligands placing their dianionic carbon donors at opposite sides of the $z$ axis and four weaker and neutral $\mathrm{N}$ donors in the $x y$ plane [49]. The spin-lattice relaxation in this anion is dominated by Raman processes at lower temperature and Orbach at higher temperatures, with two barriers of 721 and $813 \mathrm{~K}$. A similar local environment is obtained with only terminal ligands in the cation [Dy $\left.\left({ }^{\mathrm{t}} \mathrm{BuO}\right)_{2}(\mathrm{py})_{5}\right]$, which has an impressive $U_{\text {eff }}$ of above $1800 \mathrm{~K}$, and slow relaxation of its magnetization measured above $100 \mathrm{~K}$ [50]. Despite very long $T_{1}$ values persisting up to rather high temperatures in these molecules, their methyl groups may not be ideal to ensure long $T_{\mathrm{m}}$, since methyl rotations are a known source of decoherence (see section 3.1). Analogous molecules reducing this factor while maintaining long $T_{1}$ could be good qubit candidates.

Strong axiality at a Ln site can also be induced by enforcing an adequate local (strict) symmetry. This is the case in $\left[\mathrm{Yb}\right.$ (trensal)] $\left(\mathrm{H}_{3}\right.$ trensal $=2,2^{\prime}, 2^{\prime \prime}-$ 
tris(salicylideneimino)triethylamine) which has a strongly axial $C_{3 v}$ symmetry with axial anisotropy along the crystallographic 3-fold rotation axis (Fig. 5). Its spin-lattice relaxation is dominated by Raman processes rather than Orbach. The relatively small energy barrier of only $55 \mathrm{~K}$ indicates that designing magnetic anisotropy does not warrant very long $T_{1}$ [42]. While not satisfactory when seeking high temperature SMMs, this could suffice for the purpose of quantum bits, since this neutral complex still possesses a $T_{1}$ of the order $1 \mathrm{~ms}$ at $4 \mathrm{~K}$ and does exhibit a measurable quantum coherence for its $m_{J}= \pm 7 / 2$ ground doublet (see below). It could even be used as a $d=6$ qudit to allow implementation of quantum computing error correction protocols (see section 5.4) [59].

a)

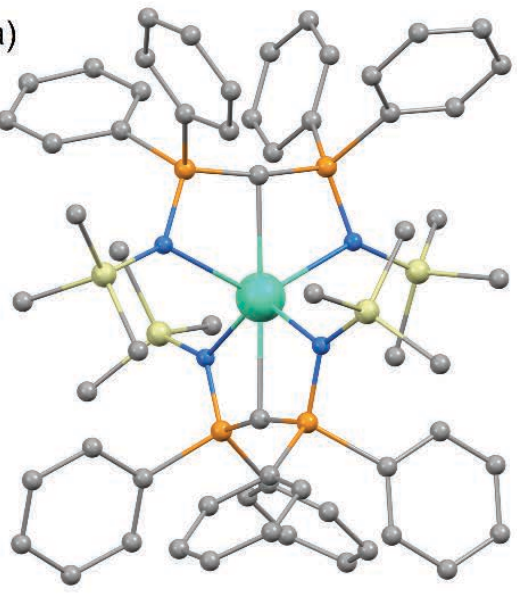

[Dy(BPIM) $]^{-}$ b)

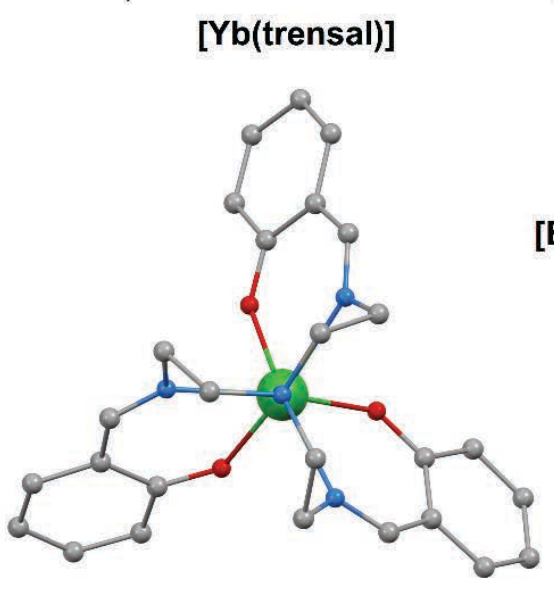

c)

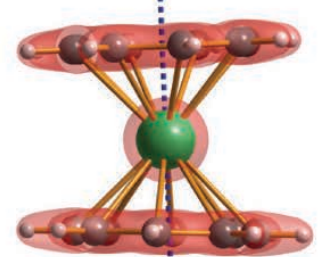

$\left[\mathrm{Er}(\mathrm{COT})_{2}\right]^{-}$

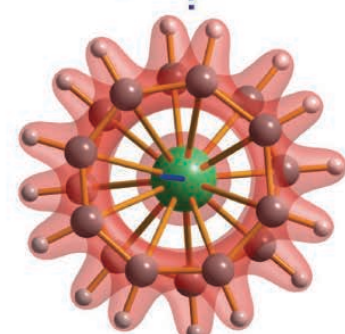

Figure 5. Structures of complexes resulting from different synthetic approaches to maximize axiality at the lanthanide as indicated (see text). Colour code: Dy, turquoise; $\mathrm{Yb}$ an $\mathrm{Er}$, green; $\mathrm{P}$, orange; $\mathrm{Si}$, yellow; $\mathrm{O}$, red; N, blue; C, grey. In the case of $\left[\mathrm{Er}(\mathrm{COT})_{2}\right]^{-}$(right; COT=cyclooctatetraene), hydrogens are also shown as light grey balls. The right part is adapted from ref. [48] with permission.

\subsubsection{Systems with high rotational symmetry ligands}


Perhaps the most efficient approach to enforce an axial ligand field at a Ln ion without implying low coordination numbers is through highly symmetric ligands forming multiple bonds with the central cation, but none exactly along the symmetry axis, the $\mathrm{N}_{4}$ donor set of phtalocyanines or porphyrins being textbook examples. Due to its large size, the Ln cation is typically sandwiched between two such ligands, forming so-called double-decker complexes. A seminal example is the homoleptic $\left[\mathrm{NBu}_{4}\right]\left[\mathrm{LnPc}_{2}\right]$ series $[32,71]$, in which the molecular symmetry is close to but not strictly $D_{4 \mathrm{~d}}$. The combined coordination modes of the phthalocyanine ligands are more adequate for oblate electronic density, leading to a much larger energy barrier to relaxation for the $\mathrm{Tb}(330 \mathrm{~K})$ than the Dy $(40 \mathrm{~K})$ analogue, in agreement with a larger separation between the \pm 6 and $\pm 5 m_{\lrcorner}$states of the former than between the $\pm 15 / 2$ and $\pm 13 / 2 m_{\lrcorner}$states of the latter. Modifications to this landmark SMM allowed significant increases of $U_{\text {eff }}$ and of the spin-lattice relaxation times. Thus, the neutral complex $\left[\mathrm{TbPc}_{2}\right]$ obtained by one-electron oxidation of the original anion has a $U_{\text {eff }}$ of $590 \mathrm{~K}$, while [TbPcPc'] heteroleptic systems with differently-substituted phthalocyanines allowed to increase $U_{\text {eff }}$ up to $938 \mathrm{~K}$ [45-46]. In the latter case, the effect was mostly ascribed to longer $\mathrm{Tb}-\mathrm{N}$ bond distances to the phthalocyanine substituted with bulky groups, bringing the $\mathrm{Tb}(\mathrm{III})$ cation closer to the other phthalocyanine and enhancing the ligand field axiality. The combination of the long $T_{1}$ of the electronic spin in [TbPc 2 , its coupling with the Tb nuclear spin and the relatively long $T_{2}$ of nuclear spin allowed to encode qudits states (see section 5.2).

Another example of heteroleptic double-decker SMMs is obtained by combining a porphyrin deck with a Kläui's tripodal capping ligand (an organometallic 
Co/tris-phosphine oxide, see below) [51]. Although the relatively low symmetry at the resulting seven-coordinate Ln site results in very modest energy barrier of only ca. $7 \mathrm{~K}$ for the Tb and Dy analogues, pulsed-EPR studies of the $\mathrm{Gd}$ analogue showed a reasonable $T_{1}$ of $43 \mu \mathrm{s}$, sufficient to allow the evaluation of the quantum coherence. Interestingly, this system was used to elegantly produce heterometallic [Ln-Ln'] complexes potentially realizing quantum gates (see section 4.2).

In agreement with predictions from ab initio calculations [62-63], the use of the higher rotational symmetry of certain planar cyclo-polydiene ligands furnishes a different approach to achieve slow relaxation. One example is the heteroleptic SMM series $\left[(\mathrm{COT}) \operatorname{Ln}\left(\mathrm{Cp}^{*}\right)\right]\left(C p^{*}=\right.$ pentamethyl cyclopentadiene $)$ [47], in which the arrangement of the $\pi$-bonding molecular orbitals of the tilted ligands in principle better suits prolate clouds such as that of the $\pm m_{\lrcorner}=15 / 2$ of $\operatorname{Er}(\mathrm{III})$, since these donors provide a predominantly equatorial ligand field. Thus $\left[(\mathrm{COT}) \operatorname{Er}\left(\mathrm{Cp}^{*}\right)\right]$ was found to have an energy barrier to spin-relaxation of $323 \mathrm{~K}$, while an homoleptic analogue $[\mathrm{K}(18 \mathrm{C} 6)]\left[\mathrm{Er}(\mathrm{COT})_{2}\right]$ had $U_{\text {eff }}$ of $286 \mathrm{~K}$ [48]. As expected the Dy(III) analogue had a significantly reduced $U_{\text {eff }}$ of only $11 \mathrm{~K}$.

Overall, the coordination geometry in double-decker type complexes, and in particular those with aromatic $p$-ligands, likely result in a relatively reduced spinphonon coupling, due to constrained Ln-ligand vibrational modes, thus reducing spin-lattice relaxation rates. The most remarkable demonstration of this is found in the dysprosocenium complex $\left[\mathrm{Dy}\left(\mathrm{Cp}{ }^{\mathrm{ttt}}\right)_{2}\right]\left[\mathrm{B}\left(\mathrm{C}_{6} \mathrm{~F}_{5}\right)_{4}\right]$, with $\mathrm{Cp}{ }^{\mathrm{ttt}}=\left(\mathrm{C}_{5} \mathrm{H}_{2}{ }^{\mathrm{t}} \mathrm{Bu}_{3}-\right.$ $1,2,4)$, which exhibits a record energy barrier of $1760 \mathrm{~K}$ and spin-lattice relaxation exceeding $400 \mathrm{~s}$ below $25 \mathrm{~K}$ and still $0.1 \mathrm{~ms}$ at temperatures as high as $112 \mathrm{~K}$ (Fig. 2) [44]. 


\subsubsection{Polyoxometallates (POMs)}

Considering the importance of tuning the local symmetry at the lanthanide ion for its spin-relaxation, polyoxometallates arise as an ideal frame to design both SMMs and qubit candidates. Indeed, the well-known regular inorganic-like structures of POMs stabilize transition metal ions, in particular Ln cations, in environments of pre-defined symmetry, while making the resulting complex chemically robust [72-74]. Materials with symmetries similar to those obtained with a phthalocyanine double-decker environment, i.e. close to $D_{4 \mathrm{~d}}$, have been studied. The best system so far is the Lindqvist-type POM $\left[\operatorname{Er}\left(\mathrm{W}_{5} \mathrm{O}_{18}\right)_{2}\right]^{9-}$, which exhibits an energy barrier to spin-relaxation of $56 \mathrm{~K}[55,58]$. In fact, only moderate energy barriers have so far been observed for Ln-POMs, in comparison with phthalocyanine systems with similar local symmetry. This shows the importance of even slight deviations from the targeted symmetry. For example, in $\left[\mathrm{Ln}\left(\mathrm{W}_{5} \mathrm{O}_{18}\right)_{2}\right]^{9-}$ compounds, the twist angle between the two planes of oxygen-donors is closer to the $45^{\circ}$ of the ideal $D_{4 \mathrm{~d}}$ symmetry than in the related $\left(\mathrm{NBu}_{4}\right)\left[\mathrm{LnPC}_{2}\right] \mathrm{SMM}$. The axial compression and axial elongation of the coordination environment, respectively, for the POMs and phthalocyanine complexes associated to this difference, result in significantly different energy level diagrams. Thus, $\mathrm{Er}(\mathrm{III})$ and $\mathrm{Tb}$ (III) POMs stabilize the $\pm m_{\lrcorner}=13 / 2$ and $m_{J}$ $=0$, states, respectively, while the ground states for the $\mathrm{Er}$ and $\mathrm{Tb}$ phthalocyanines correspond instead to $\pm m_{\lrcorner}=1 / 2$ and $\pm m_{\lrcorner}=6$, respectively. Besides this drastic effect on the actual $m_{\lrcorner}$ground state, Ln-POMs in general exhibit faster spin-lattice relaxation, which has been ascribed to smaller energy splitting values of the lower-lying crystal field sub-states [75]. 
While other local symmetries such as the $C_{5}$ environment in $\left[\mathrm{LnP}_{5} \mathrm{~W}_{30} \mathrm{O}_{110}\right]^{12-}$ or other metallates such as molybdates have allowed to describe more Ln-POM SMMs, their spin-lattice relaxation times remain only moderately long [56, 7677]. POMs nevertheless have further advantages as prospective qubit candidates, arising from the coordination through only oxygen donors, and from the relative rigidity of these clusters. Indeed, despite their fast spin-lattice relaxation, with $T_{1}$ of only $67 \mathrm{~ms}$ and $10 \mu \mathrm{s}$ at $200 \mathrm{mK}$ respectively, the $\left[\mathrm{Gd}\left(\mathrm{W}_{5} \mathrm{O}_{18}\right)_{2}\right]^{9-}$ and $\left[\mathrm{GdP}_{5} \mathrm{~W}_{30} \mathrm{O}_{110}\right]^{12-}$ POMs could be evaluated as potential qubit (see section 3.2) and qudit, respectively (see section 4.3), in both cases with a reasonable quantum coherence.

\subsection{Actinide SMMs}

While chemically similar to lanthanides, actinides differ in their relatively larger radial extension of their $5 f$ orbitals, compared with the $4 \mathrm{f}$ orbitals of lanthanides. Within coordination complexes, this typically results in a greater overlap with orbitals from ligands, causing stronger covalency. The associated large crystalfield splitting values are appealing for the design of qubit candidates. Nevertheless, to date there has not been any specific study of actinides as potential qubits. The only pulsed-EPR experiments are very recent, and were rather focused on the evaluation of covalency (see section 3.2) [78]. Although considerably more limited than that on lanthanide SMMs, there exists significant literature on actinide SMMs [35, 41], mostly on uranium materials, and in particular $\mathrm{U}(\mathrm{III})$ complexes (Table 2). This is possibly due to the large crystal splitting gaps in both the +3 and +5 oxidation states [79], and the larger $J=9 / 2$ of the former. 
The first example of a $U(I I I)$ SMM was obtained by coordination with three chelating bis-pyrazolyl-bis-phenylborate ligands, forming a neutral $\left[\mathrm{U}\left(\mathrm{Ph}_{2} \mathrm{BPz}_{2}\right)_{3}\right]$ complex that exhibits an energy barrier to spin-relaxation $U_{\text {eff }}$ of 28.8 $\mathrm{K}$ [80]. As done with lanthanides, these encouraging results were rationalized in terms of enhanced anisotropy, the axial ligand field of the trigonal prismatic environment at the $\mathrm{U}(\mathrm{III})$ cation being well-suited to the oblate-type anisotropy of the electronic cloud at the $U(I I I)$ state with a largest magnetic moment. The authors concluded that much larger relaxation barriers could be achieved by designing adequate local symmetry, and specifically favouring an axial coordination geometry. This has so far proven more elusive than expected.

Maintaining a similar trigonal local environment as in $\left[\mathrm{U}\left(\mathrm{Ph}_{2} \mathrm{BPz}_{2}\right)_{3}\right]$ but replacing the pyrazolyl $\mathrm{N}$-donors by imidazolyl coordinated through their carbon atoms (Fig. 6a) did yield a larger relaxation barrier of $47 \mathrm{~K}$ in the compound $\left[\mathrm{U}\left(\mathrm{Bc}^{\mathrm{Me}}\right)_{3}\right]$ [81]. On the contrary, two complexes with a similar pseudotetrahedral coordination environment made of either $\mathrm{O}$ or $\mathrm{N}$ donors, $[\mathrm{K}(18 \mathrm{C} 6)]\left[\mathrm{U}\left(\mathrm{OSi}\left({ }^{\mathrm{t}} \mathrm{BuO}\right)_{3}\right)_{4}\right]$ and $[\mathrm{K}(18 \mathrm{C} 6)]\left[\mathrm{U}\left(\mathrm{NSiMe}_{3}\right)_{4}\right]$, were found to have similar energy barrier to relaxation at 26 and $23 \mathrm{~K}$ respectively [82]. Another study involving $\mathrm{U}(\mathrm{III})$ complexes with different types of local symmetry and donors with significantly different ligand field strengths did not exhibit the expected drastic difference in terms of spin-lattice relaxation, with energy barriers again all in the $19-29 \mathrm{~K}$ range [83]. The first uranocene showing SMM properties, [Li(DME) $\left.)_{3}\right]\left[\mathrm{U}^{\text {III }}\left(\mathrm{COT}^{\prime \prime}\right)_{2}\right]\left(\mathrm{COT}^{\prime \prime}=\right.$ bis(trimethylsilyl)cyclooctatetraenyl dianion, Fig. 6b), also had a modest $U_{\text {eff }}$ of $27 \mathrm{~K}$ [84]. 
The number of reports on the slow spin relaxation of molecules containing $U(V)$ is even more scarce, possibly due the lower $J=5 / 2$ of this cation and its tendency to disproportionate to $\mathrm{U}(\mathrm{IV})$ and $\mathrm{U}(\mathrm{VI})$. The examples described so far have been obtained with the same capping ligand in combination with either an oxo or a nitride terminal ligand (Fig. 6c), the latter being connected to a metallacrown. Again, the observed energy barriers were all in the range 20-40 $\mathrm{K}[85-86]$.

Overall, and with still limited available experimental data at hand, neither chemical nor symmetry modifications of the $U(\mathrm{III})$ and the $U(V)$ environment seem to vary significantly their spin-lattice relaxation, leading some authors to consider that this behaviour is intrinsic to the element [83]. It is however important to stress here that contrary to lanthanides, the energy gaps between the ground and the first excited states derived by ab initio or other theoretical studies do not agree with those found experimentally, in some cases by one or even two orders of magnitude. In fact, it appears that for many uranium SMMs, the temperature dependence of the spin-lattice relaxation can be reproduced with an exponential function, as would be expected for a purely Raman twophonon process [41]. This means that the energy barriers reported so far, all using an Orbach formalism, may not be significant, and that further studies, both experimental and theoretical are still required to properly understand the spin-lattice relaxation of mononuclear actinide SMM complexes. Interestingly, the first pulsed-EPR study of actinide complexes, intended to measure the covalency of $\left[\mathrm{Ac}\left(\mathrm{Cp}^{\mathrm{tt}}\right)_{3}\right]\left(\mathrm{Ac}=\mathrm{Th}(\mathrm{III})\right.$ and $\left.\mathrm{U}(\mathrm{III}), \mathrm{Cp}^{\mathrm{tt}}=\left(\mathrm{C}_{5} \mathrm{H}_{3}{ }^{\mathrm{t}} \mathrm{Bu}-1,3\right)^{-}\right)$, did allow to properly determine $T_{1}$ values of 21 and $0.9 \mathrm{~ms}$, respectively, at 5 and $2.7 \mathrm{~K}$ for $\mathrm{Th}(\mathrm{III})$ and $\mathrm{U}$ (III) [78]. The study allowed to estimate the quantum coherence 
of these systems, thus showing that despite modestly long spin-lattice relaxation times, actinides can be considered as qubit candidates (see section 3.2).
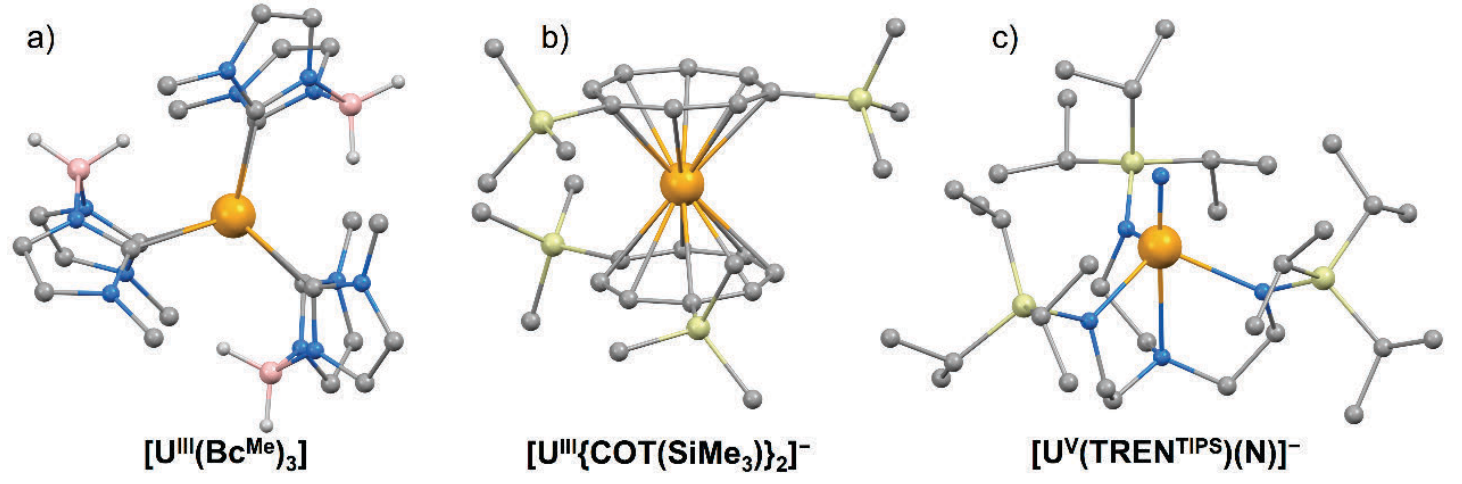

Figure 6. Molecular structures of actinide SMMs, as indicated. Colour code: U, orange; Si, yellow; N, blue; C, grey; B, light pink; H, light grey. Only hydrogens on the boron atom of $\mathrm{Bc}^{\mathrm{Me}}$ ligand are shown for clarity. 
Table 2. Characteristics of the experimental spin-lattice relaxation time $T_{1}$ for a selection of actinide monometallic Single-MoleculeMagnets, together with $T_{1}$ and phase-memory times $T_{\mathrm{m}}$ for systems studied through pulsed-EPR techniques.

\begin{tabular}{|c|c|c|c|c|c|c|c|}
\hline Complex & Local symmetry & $\mathrm{U}_{\text {eff }} / \mathrm{k}_{\mathrm{B}}{ }^{*}$ & $\max \tau @ \mathrm{~T}$ & $\max \mathrm{T}_{1} @ \mathrm{~T}$ & $\operatorname{Max} T_{\mathrm{m}} @ \mathrm{~T}$ & conditions & ref \\
\hline$\left[\mathrm{U}^{\mathrm{III}}\left(\mathrm{Ph}_{2} \mathrm{BP}_{2}\right)_{3}\right]$ & $D_{3 h}$ & 28.8 & 37 ms@1.8 K & & & & [80] \\
\hline$\left[\mathrm{U}^{\mathrm{III}}\left(\mathrm{Bc}^{\mathrm{Me}}\right)_{3}\right]$ & $C_{3 h}$ & 47 & >100 ms @ 2 K & & & & [81] \\
\hline$[\mathrm{K}(18 \mathrm{C} 6)]\left[\mathrm{U}^{\mathrm{III}}\left(\mathrm{OSi}\left({ }^{\mathrm{t}} \mathrm{BuO}\right)_{3}\right)_{4}\right]$ & $T_{\mathrm{d}}$ & 26 & $5 \mathrm{~ms} @ 1.8 \mathrm{~K}$ & & & & [82] \\
\hline$[\mathrm{K}(18 \mathrm{C} 6)]\left[\mathrm{U}^{\mathrm{II}}\left(\mathrm{NSiMe}_{3}\right)_{4}\right]$ & $T_{\mathrm{d}}$ & 23 & $5 \mathrm{~ms} @ 1.8 \mathrm{~K}$ & & & & [82] \\
\hline$\left[\mathrm{U}^{\mathrm{III}} \mathrm{I}_{3}(\mathrm{THF})_{4}\right]$ & $\mathrm{C}_{2 \mathrm{v}}$ & 19 & $\approx 4 \mathrm{~s} @ 1.8 \mathrm{~K}^{*}$ & & & & [83] \\
\hline$\left[\mathrm{U}^{\mathrm{III}} \mathrm{N}_{3}{ }_{3}\right]$ & $\mathrm{C}_{3 \mathrm{v}}$ & 29 & $\approx 1 \mathrm{~s} @ 1.8 \mathrm{~K}^{*}$ & & & & [83] \\
\hline$\left[\mathrm{U}^{\mathrm{III}} \mathrm{BIPM}^{\mathrm{IMS}}\right]$ & $C_{1}$ & 23 & $100 \mathrm{~ms} @ 1.8 \mathrm{~K}$ & & & & [83] \\
\hline$\left[\mathrm{Li}(\mathrm{DME})_{3}\right]\left[\mathrm{U}^{\mathrm{III}}(\mathrm{COT})_{2}\right]$ & $D_{8 \mathrm{~d}}$ & 27 & $\approx 200 \mathrm{~ms} @ 1.8 \mathrm{~K}$ & & & & [84] \\
\hline$\left[\mathrm{Th}\left(\mathrm{Cp}{ }^{\mathrm{ttt}}\right)_{3}\right]$ & $\approx C_{3 h}$ & & & $21 \mathrm{~ms} @ 5 \mathrm{~K}$ & $3 \mu \mathrm{s} @ 5 \mathrm{~K}$ & frozen solution & [78] \\
\hline$\left[\mathrm{U}^{\mathrm{III}}\left(\mathrm{Cp}^{\mathrm{ttt}}\right)_{3}\right]$ & $\approx C_{3 h}$ & & & $1.2 \mathrm{~ms} @ 2.7 \mathrm{~K}$ & $0.8 \mathrm{~ms}$ & frozen solution & [78] \\
\hline$\left[\mathrm{U}^{\mathrm{V}} \mathrm{O}\left(\mathrm{TREN} \mathrm{N}^{\mathrm{IIPS}}\right)\right]$ & $C_{3 v}$ & 21.5 & $\approx 13 \mathrm{~ms} @ 1.8 \mathrm{~K}$ & & & & [86] \\
\hline$\left[\right.$ Mcrown] $\left[U^{\vee} N\left(T_{R E N}{ }^{I I P S}\right)\right]$ & $C_{3 v}$ & $20-40$ & $\approx 160 \mathrm{~ms} @ 1.8 \mathrm{~K}$ & & & & [85] \\
\hline
\end{tabular}

* slow relaxation mode, in addition to a much faster mode, dominant in the case of $\left[\mathrm{U}^{\mathrm{III}} \mathrm{I}_{3}(\mathrm{THF})_{4}\right]$ 


\section{Quantum coherence of lanthanide and actinide spin-qubits}

\subsection{Causes of decoherence for spin qubits}

The main criterion to evaluate the goodness of a quantum system as qubit is its figure of merit, $Q_{M}=\Omega_{R} T_{2}$, which measures the number of feasible qubit operations before decoherence results in the loss of quantum information. $\Omega_{R}$ is the Rabi oscillation frequency and $T_{2}$ the spin-spin relaxation time. In Bloch equations, $T_{2}$ is defined as the time constant for dephasing in the $x y$ plane of the Bloch sphere, and corresponds to the rate of mutual electron spin flips. There are however other factors inducing the loss of electron spin phase coherence, and what one typically measures is a phase-memory time $T_{\mathrm{m}}$, that encompasses all of them, including $T_{2}$. Experimentally, $T_{\mathrm{m}}$ is usually measured through pulsed-EPR following the decay of an electron spin echo induced by a 2-pulse Hahn-echo sequence [39]. Since $T_{\mathrm{m}} \leq T_{2}$, only when this decay is purely single exponential, $T_{\mathrm{m}}$ can be taken as a measure of $T_{2}$. In this case, Bloch equations can be written with $T_{\mathrm{m}}$ replacing $T_{2}$. The determination of $T_{\mathrm{m}}$ is key to define the best molecular qubit candidates, and this can be done on ensembles of qubits, either in frozen dilute solutions or in diluted solids. It should be kept in mind however, that what is meaningful is $T_{\mathrm{m}}$ of the isolated qubit in the specific environment in which it will be used for quantum technologies, and its associated figure of merit $Q_{M}=\Omega_{R} T_{m}$. The spin-spin relaxation time $T_{2}$ is logically directly related with the distance, $r$, between any spin and the sensed specimen, and is expressed as $T_{2}=h r^{3} / 2 \pi \mu^{2}$ where $h$ is Planck's constant and $\mu$ is the electron magnetic moment. Clearly, isolation of the qubit spin through sufficient dilution is key to reach long $T_{\mathrm{m}}$ values. Eventually, even when decoherence sources can be supressed, $T_{2}$ (and thus 
$T_{\mathrm{m}}$ ) will always be limited by the spin-lattice relaxation, i.e. $T_{2} \leq T_{1}$, which is typically observed at relatively higher temperatures.

Besides spin-spin relaxation, the most relevant origin of decoherence is the interaction with nuclear spins, which in turn depends on the electron-nuclear dipolar coupling, the nuclear spin diffusion and the nuclear spin relaxation itself [29, 39]. Spin diffusion stands for the exchange of spin orientation energy between two neighbouring nuclear spins. Such flip-flop processes modulate the electron-nuclear dipolar interaction, thus providing a mechanism for echo dephasing. Besides reducing $T_{\mathrm{m}}$, the interaction with close-by nuclear spins also often results in modulations of the electron spin echo decay, making more difficult to model it. In approximative terms, the electron-nuclear coupling diminishes with the relative lengths of the magnetic moments while the nuclear spin diffusion decreases as the square of these magnitudes, so that the overall effect on $T_{\mathrm{m}}$ should decrease as the $3^{\text {rd }}$ power of the nuclear moment modules [29]. Experimentally, this trend has been verified, which therefore provides guidelines to maximize $T_{\mathrm{m}}$. Ligands with low amounts of nuclear spins or even nuclear-spin free should be used, and when impossible, replacement of protons by deuterium (reducing the electron-nuclear coupling by a factor of ca. 35) or $\mathrm{Cl}$ (reduction by ca. 39 for ${ }^{35} \mathrm{Cl}$ and ca. 68 for ${ }^{37} \mathrm{Cl}$ ) is a useful approach. Also, ligands with oxygen donors or sulphur donors may be preferred over those with nitrogen donors. When experiments are done in frozen solution, the use of deuterated solvents or other nuclear-spin free solvents such as $\mathrm{CS}_{2}$ can be extremely useful to maximize $T_{\mathrm{m}}$, provided that solubility allows it and the solvent or solvent mixture does form a glass at low temperature. While many RE have one or various isotope(s) with non-zero nuclear spin, these with no 
such isotopes or higher proportion of zero-nuclear spin isotopes should be preferred. However, the nuclear spin can be useful for quantum technologies, for example facilitating qudits (see sections 5.2 and 5.3) or allowing clock transitions (see section 3.2) via its interaction with the electronic spin. In this respect, isotopic enrichment of the RE may be useful and it is interesting that isotopically enriched versions of Dy(III) SMM have been recently prepared and their spin dynamics studied [87-88]. Particularly relevant is the case of [DyPc 2$]$, whose $I=5 / 2$ isotope could be used as a $d=6$ qudit (see section 5.2) [89].

Because of the large nuclear magnetic moment of protons, motion of any chemical moieties bearing protons in the surroundings of the electronic spin or in the solvent may significantly enhance the dephasing of the quantum state. This is particularly true for the rotation of methyl groups from the solvent or from within the molecular qubit. One reason is that the rotation of methyl groups already occurs at low temperatures, down to a few $\mathrm{K}$. Other motions such as rotation of a phenyl ring can also have a similar effect at higher temperatures. An additional dephasing process associated with methyl groups arises when the rate of rotation is slow compared with the electron-nuclear coupling to its protons. The couplings to the three protons become then inequivalent instead of the average observed when the rotation is fast. The associated dephasing will be then temperature dependent. Overall, methyl groups should thus be avoided, which may represent a limitation for organometallic RE systems in which for example bulky ${ }^{t} \mathrm{Bu}$ groups are very common.

There are other minor effects that can contribute to spin echo dephasing but that are not related with the design of the molecular qubit. In frozen solution, molecular tumbling upon softening of the frozen glass and librational motion of 
the anisotropic paramagnetic centre can be relevant. Also, spectral diffusion can move an excited spin outside the detection window in a pulsed EPR experiment and thus result in an additional apparent relaxation process [39].

While the foregoing can serve as a useful set of guidelines to select and/or modify potential qubit candidates among known RE SMMs, experimental studies of the real quantum coherence of any qubit candidate is necessary, due to its extreme sensitiveness to many sources of decoherence.

\subsection{Experimental estimation of quantum coherence of RE qubits}

The description of systems that are not just spin qubits but have been shown to potentially encode multiple qubit gates or qudits is given in section 5 . This includes the heterometallic [LnLn'] systems based on a porphyrin deck and Kläui's tripodal capping ligand and the metalloazafullerene $\mathrm{Gd}_{2} @ \mathrm{C}_{79} \mathrm{~N}$ (section 5.1), the phtalocyanine double and triple-decker of $\mathrm{Tb}(\mathrm{III})$ and $\mathrm{Dy}(\mathrm{III})$ (sections 5.2 and 5.3), and [Yb(trensal)] (section 5.4). The latter three involve the electronuclear states arising from coupling with the Ln nuclear spin.

\subsubsection{Lanthanide ions doped in inorganic lattices as qubits}

An early example of minimizing the decoherence of a $\mathrm{Ln}$ ions arising from both the interaction with nuclear spins and other electronic spins is given by the study of Rabi oscillation of $\operatorname{Er}($ III) ions doped into the crystalline scheelite-like salt $\mathrm{CaWO}_{4}[53,90]$. Erbium is one of the lanthanides with the least concentration of nuclear spins, with only the ${ }^{167} \mathrm{Er} /=7 / 2$ isotope being magnetic, which is present in $23 \%$ of natural abundance. More importantly, the elements forming the host lattice allow minimizing the nuclear spin bath, with 
${ }^{183} \mathrm{~W}$ being the only isotope with a nuclear spin $(I=1 / 2)$ and significant natural abundance $(14 \%)$. The $\operatorname{Er}(\mathrm{III})$ ion is inserted in the host lattice in a distorted cubic site with $S_{4}$ symmetry, resulting in a Kramers doublet ground state. For the main $I=0$ isotope, spin-echo experiments performed on a crystal with $\mathrm{Er}$ atomic concentration of $10^{-5} \%$, resulted in a $T_{\mathrm{m}}$ of the order of $7 \mu \mathrm{s}$ at $3.5 \mathrm{~K}$. As expected in the case where the dominant source of decoherence should be the spin-spin relaxation $T_{2}, T_{\mathrm{m}}$ was found to strongly depend on the concentration. Nutation experiments allowed monitoring the Rabi oscillations between the two basis states of the $\operatorname{Er}(\mathrm{III})$ spin qubit. For temperatures below $2 \mathrm{~K}$, the figure of merit $Q_{M}$ was estimated to reach values of the order of $10^{4}$, the estimated limit to allow quantum error correction codes. The exponential decay of the Rabi oscillation was however found to approach $T_{\mathrm{m}}$ only for very low power. This and the fact that $T_{m}$ also depends strongly on temperature were ascribed to additional sources of decoherence associated with inhomogeneities in the crystal field parameters, likely due to local distortions and structural defects. This shows how important it is to make truly identical qubits, in particular when working with ensembles, which in turn could be considered as one of the potential advantages of molecular spin qubits.

In a subsequent work [54], the quantum coherence of the electro-nuclear states resulting from the hyperfine interaction of the ${ }^{167} \mathrm{Er}$ isotope with its $I=7 / 2$ nuclear spin were studied in the same system, on a crystal with $0.05 \% \mathrm{Er}$ concentration, tuning the magnitude and orientation of the external magnetic field. Despite significant spin-phonon coupling, longer quantum coherence times were observed, with $T_{\mathrm{m}}$ reaching $50 \mu$ s at $2.5 \mathrm{~K}$. Also, despite that large variations of the Rabi oscillations were observed due to small deviations in the 
orientation of the applied field together with the removal of the eightfold degeneracy of $\Omega_{R}$ by the hyperfine interaction, the coherent manipulation of each electronuclear transition was demonstrated, which is required to encode a qudit.

\subsubsection{Lanthanide POMs as qubits}

The first attempt to study the potential as spin qubit of a $\operatorname{Ln}(\mathrm{III})$ molecule was reported on two $\mathrm{Gd}(\mathrm{III})$ polyoxometallates [56]. As mentioned above, one advantage of polyoxometallates is the purely oxygen donor environment, which minimizes the nuclear spin bath, although these materials often crystallize with lattice water molecules, contributing to this bath. The reason for using $\mathrm{Gd}(\mathrm{III})$ is that this ion is the only $\operatorname{Ln}(\mathrm{III})$ one with no orbital angular momentum. Therefore, its electronic level structure and magnetic anisotropy are only determined by crystal-field effects and is thus easily tuneable through changes to the local coordination. The elongated $\left[\mathrm{Gd}\left(\mathrm{W}_{5} \mathrm{O}_{18}\right)_{2}\right]^{9-}$ and bowl-shaped $\left[\mathrm{GdP}_{5} \mathrm{~W}_{30} \mathrm{O}_{110}\right]^{12-}$ polyanions (Fig. 7) were studied in powder form. Their magnetic anisotropies were determined through heat capacity, magnetic susceptibility and CW-EPR experiments. The distorted square antiprism environment close to an ideal $D_{4 \mathrm{~d}}$ symmetry in [GdW ${ }_{10}$ ] produces axial anisotropy along the molecular $z$ axis. On the contrary, in-plane anisotropy with preferential $y$ axis results from the distorted $C_{5 v}$ symmetry in $\left[\mathrm{GdW}_{30}\right]$. The expected weak magnetic anisotropy of the $\mathrm{Gd}(\mathrm{III})$ ion suffices to cause slow relaxation of the magnetization, although only at low temperatures, with $T_{1}$ of $67 \mathrm{~ms}$ and $10 \mu \mathrm{s}$, respectively, at $200 \mathrm{mK}$. This weak anisotropy in turn allows to consider using any two of the zero-field split levels for the definition of the qubit basis $|0\rangle$ and $|1\rangle$, instead of the ground 
doublet as done for most REs. Indeed, the zero-field energy gap between the two lowest lying doublets amounts to $1.06 \mathrm{~K}(22 \mathrm{GHz})$ and $0.3 \mathrm{~K}(6.3 \mathrm{GHz})$, respectively, the latter being accessible through common X-band pulsed-EPR or other microwave technologies. The phase memory time of [ $\left.\mathrm{GdW}_{30}\right]$ diluted in the diamagnetic $\left[\mathrm{YW}_{30}\right]$ analogue was determined, $T_{\mathrm{m}}$ reaching about $420 \mathrm{~ns}$ at $6 \mathrm{~K}$, with $0.1 \%$ concentration of $\mathrm{Gd}$. Both $T_{1}$ and $T_{\mathrm{m}}$ are found to increase with decreasing concentration, suggesting that decoherence through dipole-dipole interactions is still dominant, even at the lowest level of dilution used. Coherent Rabi oscillations were observed allowing to estimate a figure of merit $Q_{M}$ of at least 50. Besides, the fact that zero-field split levels are used as the qubit basis set provides a relatively large overlap of the wave-functions involved, as well as a reduced dependence on the magnetic field. In principle, this should allow tuning of the qubit without using high magnetic fields, while making the qubit operation more robust. Both aspects could be very useful to simplify the implementation of a proposed hybrid quantum computing architecture [91]. Also, further single-crystal experiments showed that all eight transitions between $m_{\mathrm{S}}$ states can be coherently manipulated with coherence times similar to those obtained from powder experiments [25]. This expands the basis of addressable states to eight, which makes the $\left[\mathrm{GdW}_{30}\right]$ molecule a potential three-qubit system (see section 4.3). 

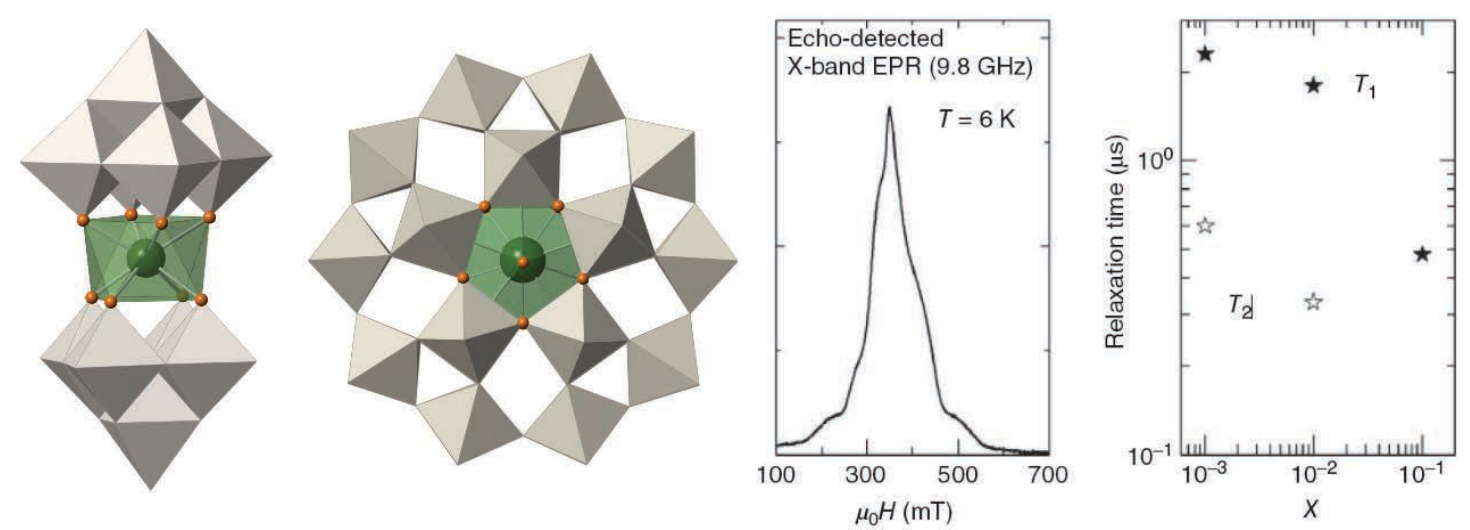

Figure 7. (Left) Molecular structures of the two families of POMs encapsulating a central Ln ion, respectively, [GdW $\left.{ }_{10}\right]$ and $\left[\mathrm{GdW}_{30}\right]$. (Right) X-band pulsed EPR at $6 \mathrm{~K}$ for powder $\left[\mathrm{Gd}_{\mathrm{x}} \mathrm{Y}_{1-\mathrm{x}} \mathrm{W}_{30}\right]:$ i) echo-detected EPR spectrum $(\mathrm{x}=0.01)$ and $\left.i i\right)$ decoherence and spin-lattice relaxation times as a function of $x$ at $\mu_{0} H=0.347$ T. Reproduced with permission from ref. [56].

Another way to reduce decoherence due to spin-spin relaxation without going to highly diluted conditions is to protect the qubit transition from its environment. An elegant, and efficient, way to do so was reported using the Ho analogue of the above $\left[\mathrm{Gd}\left(\mathrm{W}_{5} \mathrm{O}_{18}\right)_{2}\right]^{9-} \mathrm{POM}$, [HoW 10 ] [57]. A detailed study of this material[92] showed that the local symmetry close to $D_{4 \mathrm{~d}}$ produces an isolated $m_{\lrcorner}= \pm 4$ ground doublet, with the $m_{\lrcorner}= \pm 5$ as the first excited states, at about $28.8 \mathrm{~K}$. Deviations from the ideal $D_{4 \mathrm{~d}}$ symmetry result in crystal field terms that generate an unusually large quantum tunnelling gap $\Delta \approx 0.44 \mathrm{~K}(9.18 \mathrm{GHz})$ between the \pm 4 doublet, accidentally very similar to the X-band EPR frequency. Holmium has the peculiarity of having only one isotope, with $I=7 / 2$, and the combination of the crystal field and hyperfine coupling gives a series of avoided level crossings between $m_{\lrcorner}= \pm 4$ states, resulting in multiple gaps near zero field (Fig. 8). The dephasing time $T_{\mathrm{m}}$ was determined on oriented single-crystals with 
Ho concentrations from 0.1 to $10 \%$ at $5 \mathrm{~K}$ and variable fields. In all cases, sharp maxima are observed at fields close to gap minima. The longest $T_{\mathrm{m}}$ are between 5.2 and $8.4 \mu$ s for the more diluted system but remain almost the same at $1 \%$ concentration. Remarkably, the maximum $T_{\mathrm{m}}$ is still of the order of $0.6-0.7$ us in the most concentrated $10 \%$ sample. The key for such an exceptional independence to spin concentration is the extreme axial anisotropy of $\mathrm{Ho}(\mathrm{III})$ in $\left[\mathrm{HoW}_{10}\right]$ which makes it insensitive to the perpendicular applied field, while the sensitivity to variations in the axial field component vanishes when the field approaches the gap minima. This means that in the vicinity of these minima, $\mathrm{Ho}(\mathrm{III})$ is protected from any local temporal fluctuations of the magnetic field induced by dipolar decoherence sources (interactions with nuclear spin bath, spin-spin interactions, etc). This protection against the surrounding noise at the gap minima is the reason why these resonances are coined atomic clock transitions. Indeed, away from these clock transitions, $T_{\mathrm{m}}$ behaves in the usual manner, i.e. it decreases from $2-3 \mu$ s for the $0.1 \%$ dilution to already hardly 0.1 $\mu$ for the $1 \%$ dilution. A decrease of $T_{\mathrm{m}}$ at the clock transition already at $7 \mathrm{~K}$ seems to indicate that $T_{\mathrm{m}}$ is limited by $T_{1}$, which is only $20 \mu \mathrm{s}$ at $5 \mathrm{~K}$ in this system. This means that a molecular qubit with analogous clock transition but with much longer $T_{1}$ may give access to exceptionally long coherence times. 

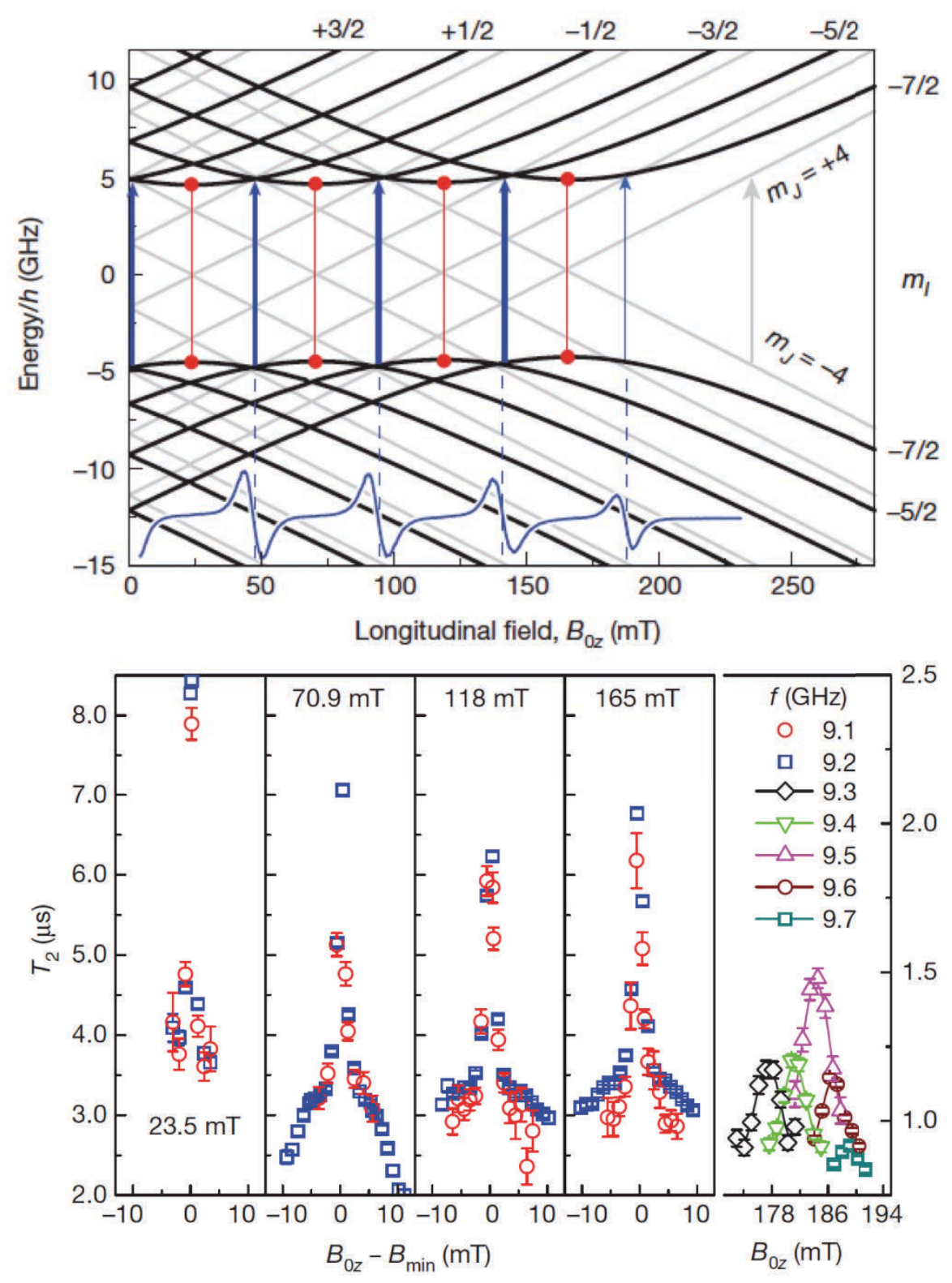

Figure 8. Zeeman diagram for the $m_{\lrcorner}= \pm 4$ and $I=7 / 2$ ground state of the $\mathrm{Ho}(\mathrm{III})$ ion in $\left[\mathrm{HoW}_{10}\right]$ with the magnetic field parallel to the $z$ axis. Black lines are for slight departure from $D_{4 d}$ symmetry. The CW-EPR spectrum is shown as inset as a full blue line, in excellent agreement with the predicted resonance positions (blue arrows). Red arrows indicate the clock transitions (top). Field dependence of the spin coherence time $T_{2}$ of a $\left[\mathrm{Ho}_{0.001} \mathrm{Y}_{0.999} \mathrm{~W}_{10}\right]$ crystal in the vicinity of the clock transitions, evidencing the divergence associated with the insensitiveness to decoherence. On the contrary, away from any of the clock 
transitions (rightmost panel), there is no such divergence in $T_{2}$ (bottom). Reproduced with permission from ref. [57].

\subsubsection{Lanthanide peptides}

Pulsed-EPR techniques have been used for some time in biology for implementing lanthanide tags to determine distances in proteins [93-94]. In that context, long coherence times is not particularly a concern. Nevertheless, DEER (double electron-electron resonance) measurements on a Gdcomplexing protein tag has recently shown $T_{m}$ of the order of $2 \mu$ s [95]. This means that Ln peptides optimized for this purpose could be considered as candidate qubits. A first study focusing along this line has recently reported the quantum coherence of Gd-LBT and Nd-LBT (LBT = lanthanide-binding peptide sequence tag, Fig. 9) to be, respectively, 2.5 and $0.6 \mu \mathrm{s}$, thus confirming this idea [69]. Calculations suggest that modifications of the binding tag peptidic sequence could allow influence the coherent properties of the complexed Ln.

\subsubsection{Organometallic actinide qubits}

As mentioned previously, a recent study of the covalency in the organometallic actinide complexes $\left[\mathrm{Ac}\left(\mathrm{Cp}^{\mathrm{tt}}\right)_{3}\right]\left(\mathrm{Cp}^{\mathrm{tt}}=\left(\mathrm{C}_{5} \mathrm{H}_{3}{ }^{\mathrm{t}} \mathrm{Bu}-1,3\right)^{-}\right)$with $\mathrm{Ac}=\mathrm{Th}(\mathrm{III})$ and $\mathrm{U}(\mathrm{III})$ (Fig. 9), used pulsed-EPR ESEEM and HYSCORE experiments, which require a detectable electron spin echo. Thus, as a collateral result, the quantum coherence of these complexes in frozen solution was determined [78]. Interestingly, the Th analogue had a measurable echo up to $150 \mathrm{~K}$, with a phase memory time $T_{\mathrm{m}}$ of $4 \mu \mathrm{s}$ below $20 \mathrm{~K}$. In comparison, the $U$ derivative showed must faster decoherence, with $T_{\mathrm{m}}$ of $0.8 \mu \mathrm{s}$ at $2.7 \mathrm{~K}$ and an electron 
spin echo only detectable below $10 \mathrm{~K}$. The difference was ascribed to drastically different electronic configurations, the Th(III) ion behaving as a spin-only $\mathrm{d}$ block $6 d^{1}$ ion. The much larger orbital extension of the $U(I I I) 5 f^{3}$ ion on the contrary gives rise to higher covalency, greater spin delocalization and stronger hyperfine interactions with the nuclear spin bath, thus favouring quantum decoherence processes. These first examples of actinide potential qubits open the door to further studies of adequate candidates.
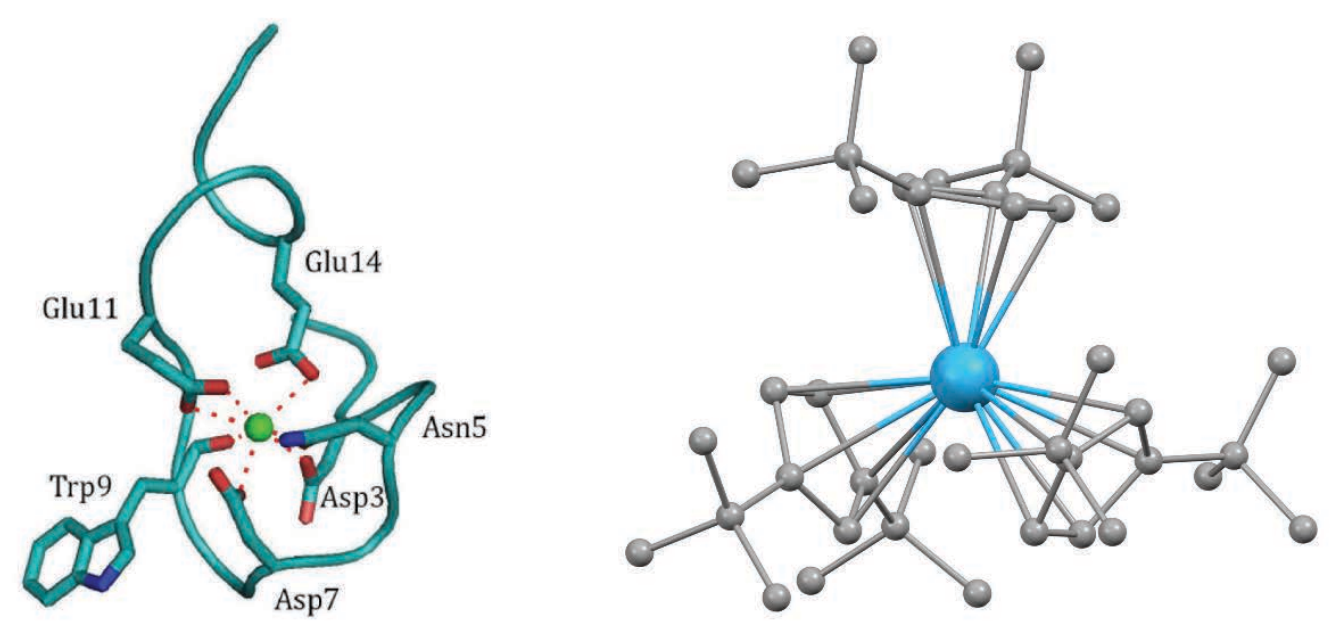

Figure 9. (Left) Molecular structures of the peptide-based qubit GdLBT and (right) the actinide qubit [Th( $\left.\left.\mathrm{Cp}^{\mathrm{tt}}\right)_{3}\right]$. 


\section{Multi-qubit quantum gates made of lanthanides}

\subsection{A dinuclear Tb(III) complex as prototype of a Two-Qubit Quantum Gate}

A universal set of quantum gates (qugates) is a small group of elementary quantum logic operations that, combined, can lead to any possible quantum algorithm, independently of its complexity. Two of the most paradigmatic universal gates are the controlled-NOT (CNOT) and the VSWAP (Fig. 10) operations. Both operate on two coupled qubits, therefore, on a computational basis formed by four double-qubit states; $|\uparrow, \uparrow\rangle,|\uparrow, \downarrow\rangle,|\downarrow, \uparrow\rangle$ and $|\downarrow, \downarrow\rangle$ (or $|1,1\rangle$, $|1,0\rangle,|0,1\rangle$ and $|0,0\rangle)$. In these $|a, b\rangle$ states, 'a' represents any of the two possible levels of the first qubit and ' $b$ ' these of the second. The CNOT operation inverts the reading of the second (or target) qubit, if and only if, the first (or control) qubit is in a specific state, previously decided by convention (for example, $|\uparrow\rangle)$. The $\sqrt{ }$ SWAP operation converts any double qubit state where both qubits are in different orientations into a superposition of both possible $|a, b\rangle$ states with $a \neq b$, ie. $(|\uparrow, \downarrow\rangle+i|\downarrow, \uparrow\rangle) /(1+i)$. 

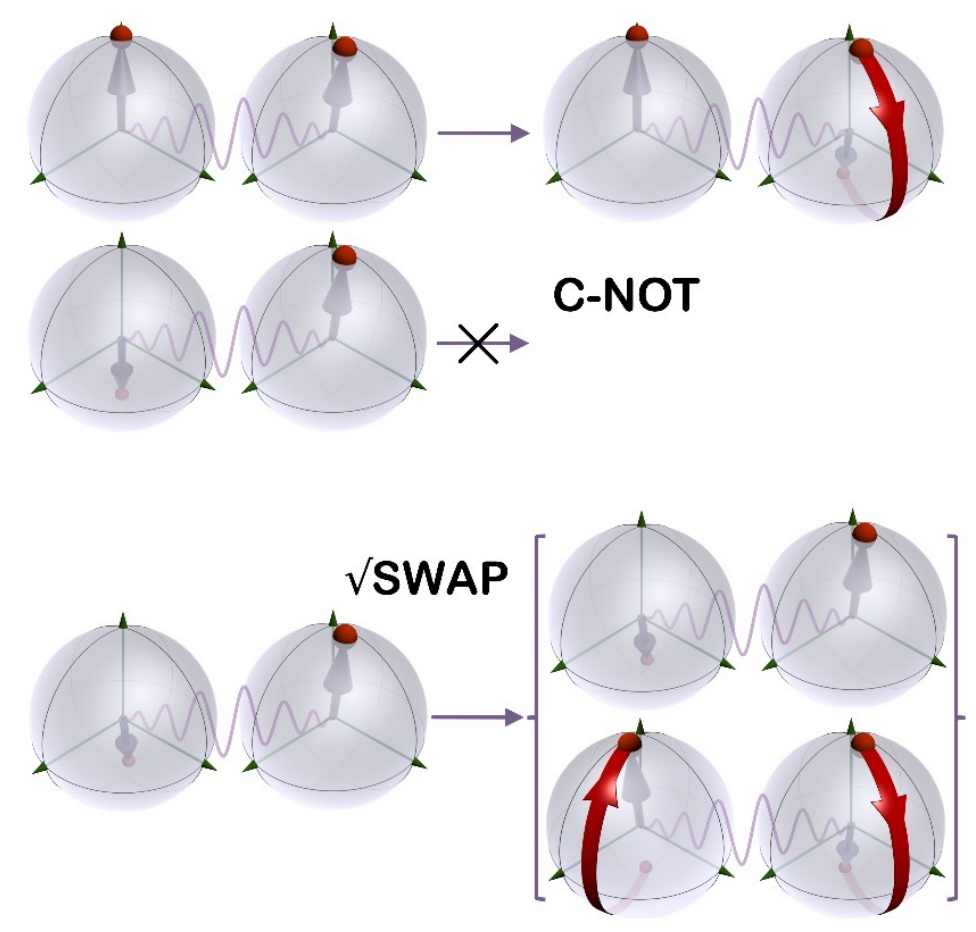

Figure 10. Representation of the two qubit quantum gates CNOT (top), which rotates the target qubit if the control one exhibits a specific value, and $\sqrt{S W A P}$ (bottom) which converts two qubits with opposite values into a superposition of the two states with both qubits in opposite values. Reproduced from ref. [24] with permission.

CNOT or VSWAP quantum gates using REs as qubits may be realized by synthesizing molecules that incorporate two inequivalent lanthanide ions with a weak mutual interaction. It is not trivial to engineer asymmetric dinuclear complexes of coupled lanthanide ions, with only very few examples present in the literature [96]. A pioneering prototype of both, a CNOT and a $\sqrt{S W A P}$ quantum gate using molecules of lanthanides was put forward with a complex of two weakly coupled $\mathrm{Tb}(\mathrm{III})$ ions exhibiting two distinct coordination environments [17, 67]. This synthesis was achieved with the bridging asymmetric ligand $H_{3} L 1$ (Fig. 11). The formation of a non-symmetric assembly 
with such type of ligand is only guaranteed if an odd number of them are disposed around the molecular axis while bridging both metals (Fig. 11). Deprotonated ligand $\mathrm{H}_{3} \mathrm{~L} 1$ was shown to form asymmetric dinuclear complexes of general formula (Hpy) $\left[\mathrm{Ln}_{2}(\mathrm{HL} 1)_{3} \mathrm{X}\left(\mathrm{H}_{2} \mathrm{O}\right)(\mathrm{py})\right]\left(\mathrm{X}=\mathrm{Cl}^{-}\right.$or $\mathrm{NO}_{3}{ }^{-}$; Fig. 11) for all possible lanthanoid ions in the +3 oxidation state [97-98].

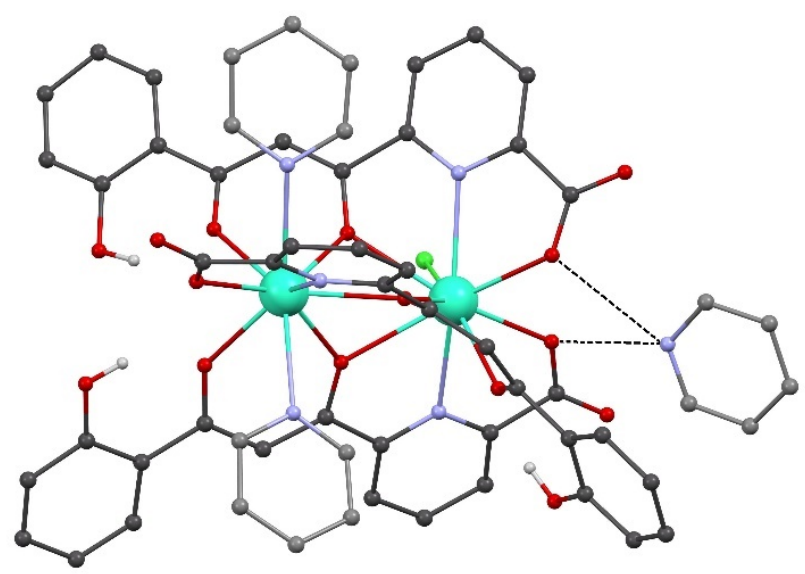<smiles>O=C(O)c1cccc(C(=O)CC(=O)c2ccccc2O)n1</smiles>

$\mathrm{H}_{3} \mathrm{~L} 1$

Figure 11. (left) Structure of the complex $(\mathrm{Hpy})\left[\mathrm{Tb}_{2}(\mathrm{HL} 1)_{3} \mathrm{Cl}\left(\mathrm{H}_{2} \mathrm{O}\right)(\mathrm{py})\right]$. Grey, $\mathrm{C}$; purple, $\mathrm{N}$; green, $\mathrm{Cl}$; red, $\mathrm{O}$; white, $\mathrm{H}$; large balls, Tb. Only hydrogen atoms from $\mathrm{OH}$ groups shown. Hydrogen bonds shown as black shaded lines. (right) molecular structure of ligand $\mathrm{H}_{3} \mathrm{~L} 1$ [97].

The $\left[\mathrm{Tb}_{2}\right]$ complex was studied with very low temperature (down to a few $\mathrm{mK}$ ) magnetization measurements under oscillating or constant external magnetic fields, together with specific heat experiments. The combined data served to establish that, $i)$ the ${ }^{7} \mathrm{~F}_{6}(L=3, S=3, J=6) \mathrm{Tb}(\mathrm{III})$ ions of the molecule are good realizations of spin-based qubits, since both exhibit an $m_{\lrcorner}= \pm 6$ ground state doublet that can be split by an external magnetic field, and that is well separated from any excited state (by ca. $180 \mathrm{~K}$ ), ii) the qubits are coupled by a weak magnetic interaction with an estimated coupling constant of $J=-0.016 \mathrm{~K}$, 
and iii) both qubits are magnetically not equivalent as revealed by the fact that the antiferromagnetic coupling does not cancel their magnetic moments, which show instead two orientations of their anisotropy axes (with an angle close to $\left.66^{\circ}\right)$. At low enough temperatures, these properties ensure the existence of an isolated $|\uparrow, \uparrow\rangle,|\uparrow, \downarrow\rangle,|\downarrow, \uparrow\rangle$ and $|\downarrow, \downarrow\rangle$ computational basis, as necessary for the operation of a double qubit quantum gate in form of four magnetic states with four different energies and not equally spaced. These are described by the Hamiltonian in equation 3, which contains the exchange coupling, the Zeeman energy of both qubits, and their hyperfine interaction, $A_{J}$, with the nuclear spin I $=3 / 2$ of the $\mathrm{Tb}$ ions.

$\widehat{H}=-2 J_{e x} \hat{J}_{1, z} \hat{J}_{2, z}+g_{1} \mu_{B} H \hat{J}_{1, z}+g_{2} \mu_{B} H \hat{J}_{2, z}+A_{J}\left(\hat{J}_{1, z} \hat{I}_{1, z}+\hat{J}_{2, z} \hat{I}_{2, z}\right)$

For clarity, only the four levels with one set of nuclear projections (eg. $m_{l, 1}=m_{l, 2}$ $=-3 / 2$ ) are shown in Fig. 12. An external magnetic field may be used to match the available wavelength with the energy necessary to cause the resonance between any pair of these four states (Fig. 12). Thus, at $H=0.07 \mathrm{~T}$ only transitions between $|\uparrow, \downarrow\rangle$ and $|\downarrow, \uparrow\rangle$ states (SWAP gate) would be resonant with radiation of $v=9.8 \mathrm{GHz}(\mathrm{X}-\mathrm{b}$ and $\mathrm{EPR})$, whereas a magnetic field of $H=0.28 \mathrm{~T}$ creates the appropriate splitting to generate transitions between the states $|\uparrow, \uparrow\rangle$ and $|\uparrow, \downarrow\rangle$ (CNOT gate) using the same resonance energy. The initialization of the system may be brought about by cooling the system (with $99.3 \%$ of the ground state populated at $T=0.1 \mathrm{~K}$ ) since frequency dependent alternating field magnetization data show that the spin-lattice relaxation time $\left(T_{1} \approx 4 \times 10^{-4} \mathrm{~s}\right)$ allows for rapid relaxation. The transitions associated to the SWAP and the CNOT quantum gates are allowed, perhaps by the presence of weak transverse 
anisotropy fields, as witnessed by two broad continuous-wave EPR signals measured on powdered samples.
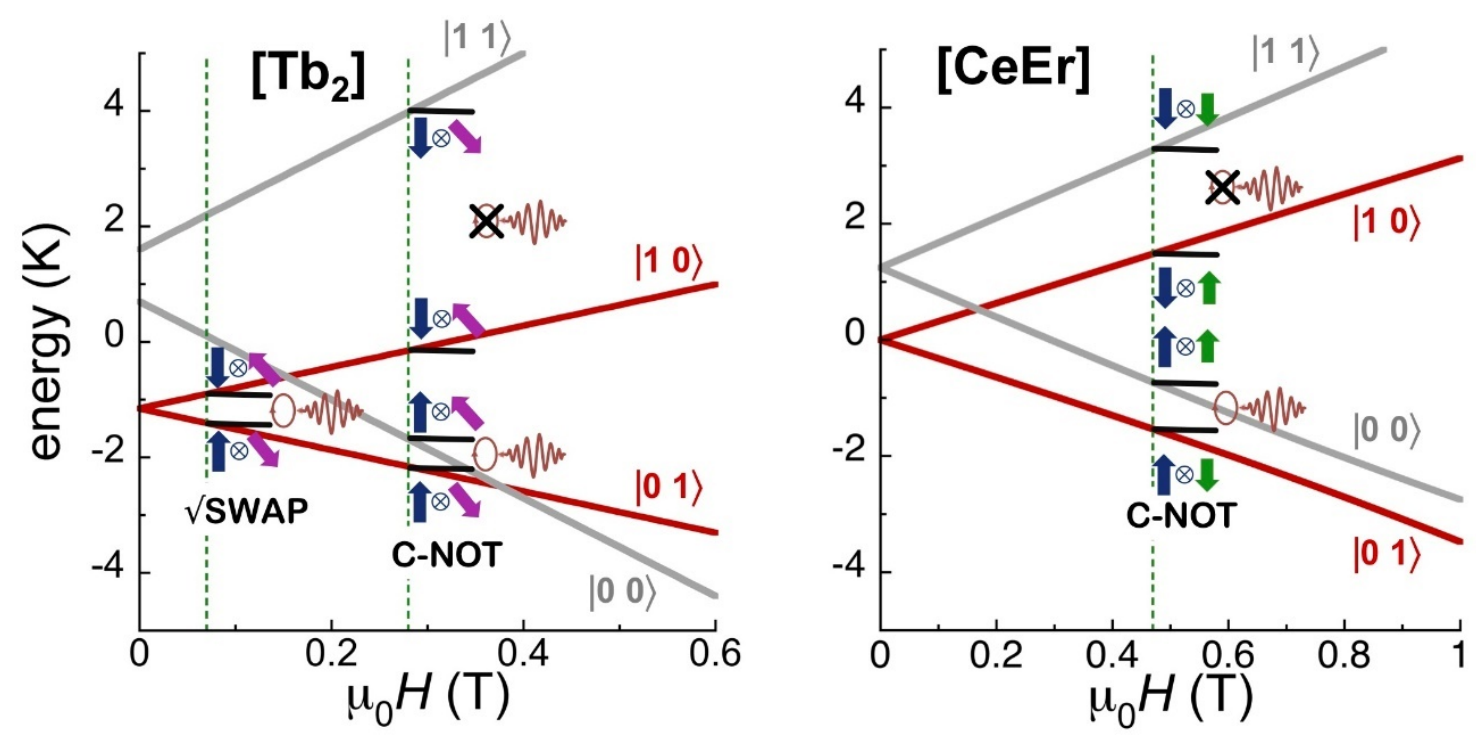

Figure 12. (Left) Lowest energy levels of $(\mathrm{Hpy})\left[\mathrm{Tb}_{2}(\mathrm{HL} 1)_{3} \mathrm{Cl}\left(\mathrm{H}_{2} \mathrm{O}\right)(\mathrm{py})\right]$ as a function of the magnetic field calculated with the parameters that result from diagonalization of Equation 3, only for the set of nuclear projections with $\mathrm{m}_{l, 1}=$ $m_{l, 2}=-3 / 2$ [67]. Resonances caused by X-band EPR radiofrequency excitation corresponding to the CNOT and $\sqrt{S W A P}$ qugates are indicated. (Right) The analogous energy spectrum for $(\mathrm{Hpy})\left[\mathrm{CeEr}(\mathrm{HL} 1)_{3}\left(\mathrm{NO}_{3}\right)\left(\mathrm{H}_{2} \mathrm{O}\right)(\mathrm{py})\right]$, obtained by fitting the experimental data to the parameters of Equation 5, showing the resonance of the CNOT at the X-band frequency [68]. Adapted from ref. [24] with permission.

\subsection{Heterometallic Lanthanide Complexes as Quantum Gates}

The potential of using dinuclear Ln complexes as 2-qubit qugates would be much more versatile if heterometallic species were accessible. Given the similar reactivity of most lanthanides, it is an outstanding synthetic challenge to prepare 
pure heterometallic molecular compounds with two or more different lanthanides [99]. This goal usually requires synthetic procedures based on the sequential incorporation of the different metal ions into the discrete assembly [100-109], or by linking preformed moieties, containing different metals [110111]. These preparation methods are however quite lengthy. Alternatively, the synthesis of pure heterometallic Ln compounds from one-pot reactions with multitopic ligands has been achieved by exploiting the size difference existing between any two different $4 \mathrm{f}$ metal ions [112]. These methods however, often produce metal distributions deviating to varying extents from statistical compositions [99, 113-116].

A recent and very elegant procedure to produce heterometallic [Ln-Ln'] complexes consists of generating mononuclear $\operatorname{Ln}(\mathrm{III})$ complexes coordinated by a porphyrin and Kläui's tripodal capping ligand (an organometallic Co/trisphosphine oxide) with the porphyrin bearing both, an ethynyl and a second substituent. Dinuclear Ln(III) complexes (Fig. 13) are then obtained by the oxidative coupling of two monomers through their respective ethynyl groups. Coupling monomers of two different metals and with the second substituent of their porphyrin ligands also different produces statistical mixtures of dinuclear Ln-Ln, Ln-Ln' and Ln'-Ln' dimers that can be easily separated by chromatography [51]. In this manner, $\left[\mathrm{Gd}_{2}\right],\left[\mathrm{Tb}_{2}\right],\left[\mathrm{Dy} \mathrm{y}_{2}\right]$ and [Dy-Tb] complexes were prepared and studied. The purity of the heterometallic analogue was established unambiguously using Matrix Assisted Laser Desorption IonizationTime of Flight (MALDI-TOF) mass spectrometry. The bulk magnetization properties of compounds $\left[\mathrm{Tb}_{2}\right],\left[\mathrm{Dy}_{2}\right]$ and $[\mathrm{Dy}-\mathrm{Tb}]$ was investigated, suggesting the presence of weak magnetic interactions between $\operatorname{Ln}(\mathrm{III})$ centers within these 
molecules. Such behavior turns the heterometallic analogue into a potential prototype of a two-qubit quantum gate (with two distinct, coupled qubits). The three compounds display slow relaxation of the magnetization (witnessed by frequency dependent signals of the out-of-phase component, $x^{\prime \prime}$, of the magnetic susceptibility under an oscillating magnetic field) only in the presence of a constant external magnetic field of $2 \mathrm{kOe}$. Of particular relevance are the studies of quantum coherence performed on the allowed EPR transitions within the $\pm m_{\lrcorner}$multiplet of the ${ }^{8} S_{7 / 2}$ ground state of $\mathrm{Gd}(\mathrm{III})$ for the complex $\left[\mathrm{Gd}_{2}\right]$ in comparison with the mononuclear precursor. Thus, the phase memory time, $T_{M}$, decreases with cooling for both, the dinuclear and the mononuclear compound, with best values in the 3.0 to $3.6 \mu$ s range at $2 \mathrm{~K}$, as detected using Hahn-echo sequences of pulsed X-band EPR. Specially relevant is the observation that the coherence time of each $\mathrm{Gd}(\mathrm{III})$ ion of the dinuclear compound is only slightly affected by the presence of the other metal center of the molecule.

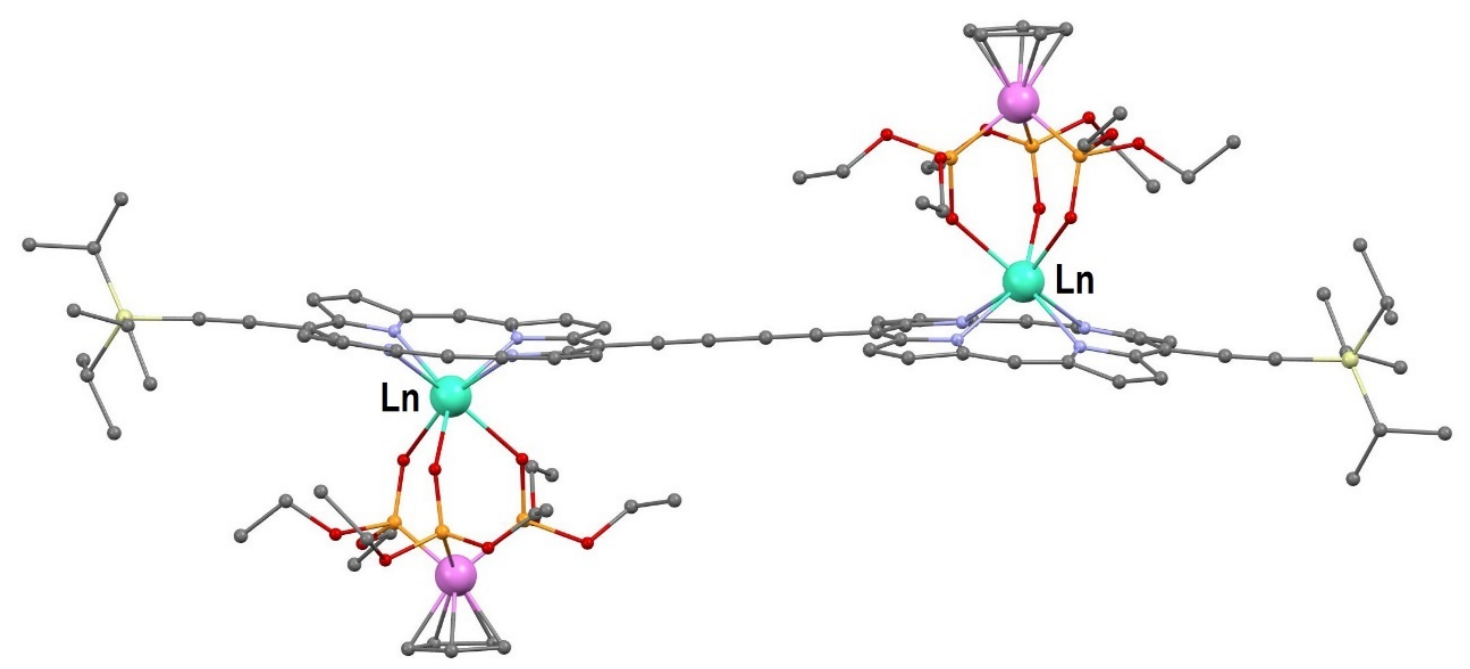

Figure 13. Representation of bis-porphyrinato [ $\left[\mathrm{n}_{2}\right]$ complexes [51] with capping Kläui's tripodal capping ligands. Grey, C; red, O; orange, P; yellow, Si; 
purple, N; green, Ln; violet, Co. Hydrogen atoms and bis-tert-butylphenyl substituents on the porphyrins not shown for clarity.

For some time, heterometallic Ln complexes have also been accessed as tripledecker complexes formed by two metals sandwiched between a central phthalocyanine-type ligand and two peripheral ones. The original triple-decker complexes where homometallic [117-119]. In a pioneering work, the Ln $\cdots$ Ln magnetic interaction was investigated within a family of triple-decker dinuclear complexes, by comparing their magnetic susceptibility with that of the corresponding [Ln-Y] analogue [100]. All compounds were prepared by a reaction of a preformed $\left[\mathrm{Ln}(\mathrm{Pc})_{2}\right]$ complex $(\mathrm{Pc}=$ phthalocyanine $)$ with a second lanthanide complex, [Ln'(acac) $)_{3}$ (acac=acetylacetonate) and a substituted phthalocyanine, $\mathrm{Pc}^{*}[107,120]$. This methodology has recently been exploited to prepare the $[\mathrm{Dy}-\mathrm{Tb}]$ analogue $\left[\mathrm{PcDyPcTbPc}^{*}\right]$ with the goal of studying the complex as the realization of a molecular 2qubit quantum gate [104]. Here, as in the previous system, an approximation to the individual properties of the isolated qubits is accessible thanks to the availability of the corresponding mononuclear, double decker complexes $\left[\operatorname{Ln}(P c)_{2}\right]$. Susceptibility measurements on the $[\mathrm{Dy}-\mathrm{Tb}]$ analogue unveil a magnetic interaction between both qubits, which is confirmed by specific heat data. Dynamic studies confirm the slow relaxation of the magnetization within the dinuclear molecule, also demonstrating that this behavior is different than that resulting from the added effect of the isolated individual qubits. Quantum coherence properties are not available for this system. Nevertheless, and very importantly, the molecular quantum gate prototype was deposited on gold or highly oriented pyrolytic 
graphite (HOPG) surfaces and X-ray absorption spectroscopy and magnetic circular dichroism (XAS and XMCD) corroborated that the integrity and the magnetic properties of the molecules were maintained on these substrates.

An additional family of dinuclear heterometallic [LnLn'] molecules features diamonds made of two hydroxide bridged metals, sandwiched within two polyoxometalate (POM) ligands, as described by the formula $\mathrm{TBA}_{8} \mathrm{H}_{4}\left[\mathrm{LnLn}(\mathrm{OH})_{2}\left(\mathrm{Y}-\mathrm{SiW}_{10} \mathrm{O}_{36}\right)_{2}\right]$ [108]. Mononuclear complexes featuring one lanthanide ion inserted in between two POM ligands had been previously studied as potential qubits, exhibiting interesting coherence values (see above) [56]. The heterometallic dinuclear POMs are then possible 2qubit quantum gates. These molecules are prepared first, through the formation of a mononuclear sandwiched complex with the second metal site vacant, occupied instead by hydrogen bonded $\mathrm{H}_{2} \mathrm{O}$ molecules. Subsequently, the monometallic complex is made react with $\left[\mathrm{Ln}(\mathrm{acac})_{3}\right]$ ( $\mathrm{Ln}$ being any lanthanide of choice) to obtain the desired [LnLn'] combination. The authors of this work studied the influence of the second lanthanide on the slow relaxation properties of the Dy(III) ion. They concluded that the influence was the result of the structural effects derived from the changing size of the variable metal, affecting the axial character of the ligand field around Dy.

The structure of the comprehensive family of homometallic $\left[\mathrm{Ln}_{2}\right]$ complexes described in the previous section (Fig. 11) was analyzed in detail since, for the entire period of REs, they were amenable to single crystal X-ray diffraction studies (SCXRD). From this analysis emerged, for the various structural parameters, the trend expected from the lanthanide contraction phenomenon [98]. The most remarkable observation is, however, that for all the molecules of 
this series, one metal location always features larger metal-donor distances than the other, which suggests that in the molecular architecture, one position should favor larger metals than the other. This opened up the prospect of preparing heterometallic analogues for any (Ln,Ln') combination, since any pair of different lanthanide ions differ in their ionic radius. This was proven experimentally and through $a b$ initio calculations, with a large number of examples [121-122]. The system was then exploited for the design of an improved prototype of a 2qubit quantum gate, embodied by the [CeEr] derivative (Hpy)[CeEr(HL1) $\left.)_{3}\left(\mathrm{NO}_{3}\right)\left(\mathrm{H}_{2} \mathrm{O}\right)(\mathrm{py})\right]\left(\mathrm{H}_{3} \mathrm{~L} 1\right.$ is depicted in Fig. 11) [68]. This combination was chosen because both Ln(III) centers are Kramers' anions with a doubly degenerate ground state resulting from the crystal field, very different from each other $\left({ }^{2} F_{5 / 2}\right.$ and ${ }^{4} I_{15 / 2}$ ground states for $\mathrm{Ce}$ and $\mathrm{Er}$, respectively, with gyromagnetic ratios of $g_{J}=6 / 7$ and $\left.g_{J}=6 / 6\right)$, the splitting of which may be tuned with an external magnetic field, thus constituting good definitions of qubits. In addition, while the Ce ions do not feature any nuclear spin, only $22.9 \%$ of the $\mathrm{Er}$ isotopes have a magnetic nucleus. The reduced presence of nuclear spins contributes to minimize coherence times. The great versatility of this synthetic method facilitates the possibility of studying each individual qubit of the [CeEr] ensemble in the exact same environment that it occupies within the quantum gate, without the influence of the other qubit. This was done through the synthesis of the $[\mathrm{CeY}]$ and [ $\mathrm{LaEr}]$ derivatives, where the diamagnetic ions $\mathrm{Y}(\mathrm{III})$ and $\mathrm{La}(\mathrm{III})$ occupy the small and large position within their respective molecules. From magnetization and EPR studies, it was demonstrated that at sufficiently low temperature $(T \leq 3 \mathrm{~K}$ for $[\mathrm{LaEr}]$ and $T \leq 7$ $\mathrm{K}$ for [CeY]), both qubits behave as anisotropic, effective spin-1/2 systems, with 
very different gyromagnetic tensors, $\hat{g}_{i}$ (thus, being magnetically inequivalent), according to the following Hamiltonian (where $i=1,2$ refer to $\operatorname{Er}(\mathrm{III})$ and $\mathrm{Ce}(\mathrm{III})$ ions, respectively).

$\widehat{H}_{i}=-\hat{g}_{i} \mu_{B} H \hat{S}_{i}$

These data were compared with results from the same experiments on the [CeEr] qugate, which, together with specific heat determinations served to demonstrate the existence of a weak magnetic interqubit coupling. These properties confer to the heterometallic cluster the requirements to act as a 2qubit quantum gate. Its operation can be described by the Hamiltonian below.

$\widehat{H}_{12}=-\hat{g}_{1} \mu_{B} H \hat{S}_{1}-\hat{g}_{2} \mu_{B} H \hat{S}_{2}-\frac{1}{g_{J 1} g_{J 2}} J_{12} \hat{S}_{1} \hat{g}_{1} \hat{g}_{2} \hat{S}_{2}$

The eigenstates of this Hamiltonian are the four possible combinations emerging from considering two possible states for each qubit, thus constituting a four-dimensional Hilbert space $(|1,1\rangle,|1,0\rangle,|0,1\rangle$ and $|0,0\rangle)$ that furnishes the computational basis for a 2qubit quantum gate. Under an external magnetic field, all possible transitions within this four-level energy spectrum are nondegenerate, thus, allowing the use of a customized radiofrequency (for example, X-band photons) to realize a given quantum logic operation, such as the CNOT qugate (Fig. 12). In this case, this can be realized with the application of a magnetic field with $\mu_{0} H=470 \mathrm{mT}$, which facilitates the resonance between the $|0,1\rangle \rightarrow|0,0\rangle$ transition using $X$-band radiation. The quantum coherence of this transition was evaluated by means of pulsed EPR, with an estimated $T_{2}$ decoherence time of 410 ns.

\subsection{Codification of several qubits within one Lanthanide center}


The 2qubit quantum gates described above exploit the Hilbert space generated by two coupled two-level spin systems (qubits) as a suitable computational basis. In the same manner, the realization of a $\mathrm{N}$-qubit quantum gate utilizes any $2^{N}$ space from a proper quantum system. For example, a three-qubit quantum gate, such as the so called TOFFOLI operates on a space made of eight states, which encode the different ways in which these three qubits may combine $(|0,0,0\rangle,|0,0,1\rangle,|0,1,0\rangle,|1,0,0\rangle,|1,1,0\rangle,|1,0,1\rangle,|0,0,1\rangle$ and $|1,1,1\rangle)$. The TOFFOLI qugate, also called controlled-CNOT (CCNOT), inverts the state of the target qubit if the other two (control qubits) are in a specific state, for example, 0$\rangle$. It is then a CNOT that only operates under an additional first control, enabling only the transition $|0,0,0\rangle \rightarrow|0,0,1\rangle$, of all the possible conversions within the Hilbert space. This can thus be realized by three different coupled two-level quantum systems (three different, coupled qubits) but also by a suitable system exhibiting eight quantum states. This can only be done on the condition that all the transitions between any two states of this basis be different in energy. It has been recently shown physically that the eight states of a three qubit system may be mapped by the spin states of the ${ }^{8} S_{7 / 2}$ term of only one Gd(III) center [25]. The spin system of gadolinium in the ${ }^{8} S_{7 / 2}$ state features eight non-equally spaced states if the metal center experiences a crystal field that causes magnetic anisotropy, which allows addressing each transition independently. The case of $\mathrm{Gd}(\mathrm{III})$ is ideal since the energy splitting of the spin octet caused by the crystal field is sufficiently small to maintain the transitions between them within a range accessible by microwave technologies. These properties were demonstrated on a complex made of a $\mathrm{Gd}(\mathrm{III})$ center encapsulated inside a polyoxometalate cage, with formula 
$\mathrm{K}_{12}\left[\mathrm{Gd}\left(\mathrm{H}_{2} \mathrm{O}\right) \mathrm{P}_{5} \mathrm{~W}_{30} \mathrm{O}_{110}\right]$ (abbreviated as $\mathrm{GdW}_{30}$ ) [25]. The energy spectrum of this ion is described by the Hamiltonian below.

$\widehat{H}=D\left[S_{z}^{2}-\frac{1}{3} S(S+1)\right]+E\left(S_{x}^{2}-S_{y}^{2}\right)-g \mu_{B} \hat{S} \vec{H}$

This Hamiltonian incorporates the magnetic anisotropy introduced by the crystal field and indeed furnishes eight non-degenerate spin states. These states were detected by continuous wave X-band EPR spectroscopy (on powder and single crystals) through the seven allowed transitions between adjacent $m_{ \pm J}$ levels (Fig. 14). The chemical versatility of this system allows to disperse the $\mathrm{Gd}$ complexes within an isostructural diamagnetic matrix of analogous complexes of $Y(I I I)$, yielding a solid solution with composition $\mathrm{Y}_{0.99} \mathrm{Gd}_{0.01} \mathrm{~W}_{30}$. The dispersion strongly reduces dipolar interactions between $\mathrm{Gd}(\mathrm{III})$ centers in the solid, thus minimizing the decoherence. The spin dynamics of this complex were studied on the diluted sample by pulsed EPR. Hahn echo sequences were employed to show that the seven transitions exhibit quantum coherence and that the phase memory times are in the 470 to 600 ns range. Rabi oscillations for all the transitions were determined via spin nutation experiments and the dependence with the radiation power and the magnetic field of the frequency of these oscillations, $\Omega_{R, n}(n=1-7$; each of the allowed transitions), established. The CCNOT quantum gate would be realized with a single pulse causing a $\pi$ rotation $R_{1}(\pi)$ tuned at the transition $|0,0,0\rangle \rightarrow|0,0,1\rangle$ (transition $n=1$ in Fig. 14). More complicated operations may also be realized through complex pulse sequences. The creation of the Greenberger-Horne-Zeilinger state, $|\Psi\rangle=$ $(1 / \sqrt{2})(|0,0,0\rangle+|1,1,1\rangle)$, which is a maximally entangled state of three qubits, can be done applying to the ground state, $|0,0,0\rangle$, the sequence $R_{1}(\pi / 2) R_{2}(\pi) R_{3}(\pi) R_{4}(\pi) R_{5}(\pi) R_{6}(\pi) R_{7}(\pi)$. In this sequence, $R_{n}(\theta)$ is a rotation by 
an angle $\theta$ tuned at transition $n$ among the seven shown in Fig. 14. This work shows that only one gadolinium center may provide the hardware to operate up to three qubits. It is conceivable that molecules connecting more than one Gd(III) center may serve to implement a larger number of qubits. For example, the $\left[\mathrm{Gd}_{2}\right]$ version of the quantum gate prototypes shown in sections 4.1 and 4.2 could constitute a molecular device embedding a total of six qubits.
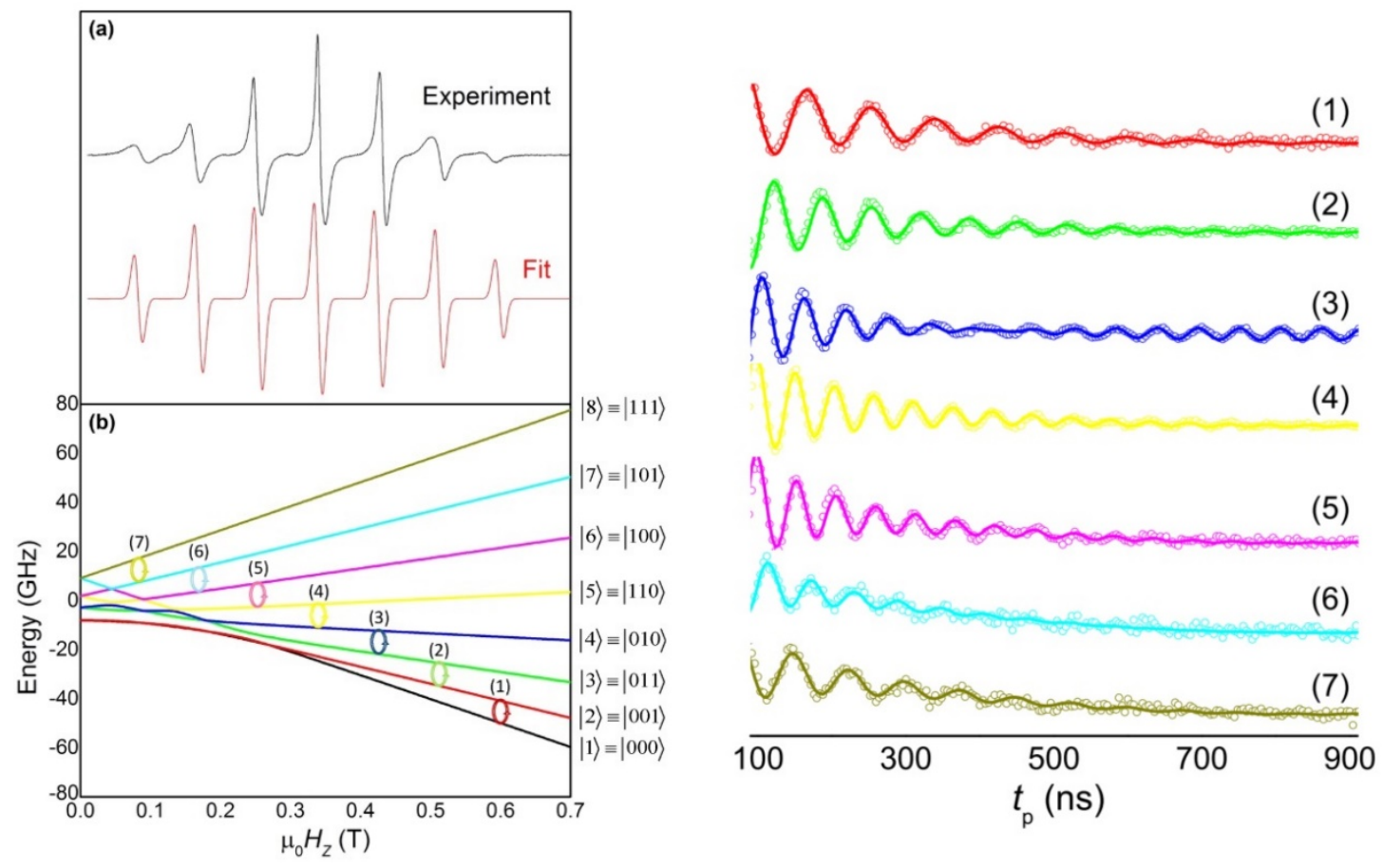

Figure 14. (a) Continuous wave X-Band EPR spectrum of a single crystal of $\left[\mathrm{GdW}_{30}\right]$ doped within a matrix of [YW $\mathrm{YW}_{30}$. (b) Energy diagram of the $S=7 / 2$ spin ground state of $\mathrm{Gd}(\mathrm{III})$ as a function of $H_{z}$, showing the eight $m_{S}$ levels $( \pm 7 / 2$, $\pm 5 / 2, \pm 3 / 2, \pm 1 / 2$ ) as the possible basis of three qubits, indicating the seven $\Delta m_{S}$ $=1$ transitions among these levels. (c) Rabi oscillations of these seven transitions. Circles are experimental data and lines are least-square fits [25].

\section{Realization of quantum algorithms with lanthanide qudits}




\subsection{Lanthanide electronic spins as qudits}

The minimum possible amount of quantum information is encoded by a qubit, which exhibits two well defined quantum states. However, if the $n$ accessible states of a given system is larger than two, it can be exploited as a higher dimension qudit. In this sense the $\mathrm{GdW}_{30}$ "three-qubit" system described in the previous section constitutes a qudit of dimension $d=8$. Exploiting large dimension qudits enhances the density of quantum information that can be handled within a physical system, allowing it to perform more complex tasks. For example, the single ion $\mathrm{GdW}_{30}$ molecule possesses the minimum dimension necessary to correct a single amplitude or shift phase error [123]. In this context, a dimer of two $G d(I I I)$ ions encapsulated into the paramagnetic $(S=1 / 2)$ azafullerene $C_{79} \mathrm{~N}$ and strongly coupled to it [124], with a total spin $S=15 / 2$ has been proposed as $d=16$ qudit [52]. The dimension of this qudit, termed $\mathrm{Gd}_{2} @ \mathrm{C}_{79} \mathrm{~N}$, arises from the ferromagnetic coupling between the $S=7 / 2 \mathrm{Gd}(\mathrm{III})$ ions and the spin of the cage. It is suggested that the cage acts to protect the spin wavefunction from environmental noise, thereby increasing its quantum coherence. Continuous wave X- and Q-band EPR spectroscopy unveils the 16fold level structure of the ground state multiplet, thanks to the anisotropy of the spin ground state. The non-degeneracy of the fifteen allowed transitions of this multiplet is necessary for the quantum system to operate as qudit. Simulations of the spectra considering the zero field-splitting for the observed crystallographic symmetry showed that all the transitions are accessible individually within a relatively narrow energy range, ideal for their practical quantum manipulation. This was investigated using pulsed X-band EPR experiments, which revealed quantum coherence, detected over a broad range 
of magnetic fields in the 2 to $15 \mathrm{~K}$ temperature range. The measured $T_{1}$ values were found in the order of microseconds. The feasibility of coherent manipulations of the quantum states of the $S=15 / 2$ multiplet was examined via echo-detected nutation experiments performed at $5 \mathrm{~K}$. They showed the existence of Rabi cycles within the entire relevant range of magnetic fields. The latter allows selecting the distinctive transitions of the multiplet with Rabi frequencies that can be calculated using the parameters deduced from fitting the cw-EPR spectra.

\subsection{Qudits encoded into the Lanthanide nuclear spin}

It has been suggested for some time that one way of addressing the concern posed by the short coherence times of electronic spin wave functions is turning to nuclear spins [125]. Indeed, the latter are expected to exhibit larger $T_{\mathrm{m}}$ and $T_{2}$ times because they are much better protected from the environment. This increases however, the challenge of addressing them from the exterior. It was recently proposed that the four $\pm m_{1}$ states $(|+3 / 2\rangle,|+1 / 2\rangle,|-1 / 2\rangle$ and $|-3 / 2\rangle)$ of the $I=3 / 2$ nuclear spin of $\mathrm{Tb}(\mathrm{III})$ within the complex $\left[\mathrm{Tb}(\mathrm{Pc})_{2}\right]$ (Fig. 15) could be used to encode a $d=4$ qudit.[126-127] It was shown that the state of the projection $\hat{I}_{z}$ can be read through its effect on the $m_{J}= \pm 6$ electronic spin ground state of this ion. Indeed, the hyperfine interaction between $\hat{I}$ and $\hat{J}(A$ in the Hamiltonian $\left.\widehat{H}_{H F}=A \hat{I} \hat{J}\right)$ splits both states of the $m_{J}= \pm 6$ doublet into four nonequally spaced levels. The Zeeman splitting of all these states caused by the influence of an external magnetic field generates four avoided energy crossings, where $m_{l}$ is conserved, while $m_{\lrcorner}$changes sign (Fig. 15). Therefore, the probability of crossing between $m_{\lrcorner}=+6$ to $m_{\lrcorner}=+6$ and vice versa at these 
points is enhanced dramatically through quantum tunneling (enabled by offdiagonal terms of the ligand field Hamiltonian) and can be calculated with the Landau-Zener (LZ) formula, as $P_{\mathrm{LZ}}$ [128]. As a result, if the magnetic field is swept across the range containing the avoided crossings, transitions among both $m_{\lrcorner}= \pm 6$ spin states accelerate when this field matches that of the crossings. This was in fact demonstrated on a single-molecule device consisting on one molecule of $\left[\mathrm{Tb}(\mathrm{Pc})_{2}\right]$ trapped in between two gold nano-electrodes [126]. This complex contains one unpaired electron localized at the $\pi$-system of the Pc ligands that is tunnel-coupled to the electrodes creating a quantum dot. The transport of current through this system leads to a weak spin $S=1 / 2$ Kondo effect [129]. Since the unpaired electron of the molecule is coupled ferromagnetically to the electronic spin of the $\mathrm{Tb}(\mathrm{III})$ ion, the transport properties are sensitive to the state of $\hat{J}_{z}(+$ or -6$)$. Thus, as the magnetic field is swept back and forth, the fields of the avoided crossings are sensed as conductance jumps caused by the enhancement of the spin reversal processes at these crossing points (Fig. 15). The fields singled out from the transport measurements are in perfect agreement with these predicted for the avoided crossings [71] and coincide with the fields where QTM is observed via magnetization measurements, which depend on the nuclear spin state. This provides a way of electrically reading-out the nuclear spin state of a single molecule simply by measuring current transport while varying the magnetic field. This phenomenon can also be used to initialize the system; if one desires to prepare the qudit into a given nuclear spin state $m_{l}$, the magnetic field may be scanned in both directions, until the jump in conductivity corresponding to this $m_{l}$ state occurs. At this moment, the system is ready at the predetermined state 
and poised for quantum operations. The next step is the coherent manipulation of the nuclear spin. This can be done by exploiting the hyperfine Stark effect, which is the influence on the hyperfine constant $A$ by an external electric field [130]. Since $A$ may be expressed in terms of an effective magnetic field, $B_{\text {eff, }}$ at the center of the nucleus, an oscillating modulation of $A$ induces a periodic modulation of the magnetic field, which can cause the rotation of the nuclear spin. This can be done by application of a microwave (MW) pulse of duration $\tau$. The realization of such experiments a various $\tau$ values revealed Rabi oscillations. The dephasing coherence time $T_{2}$ of the nuclear spin in this device was found to be $T_{2}=64 \mu \mathrm{s}$. The latter was measured from the Ramsey interference fringes obtained by applying two MW pulses separated by a delay time $\tau$. Once a specific state is created, it can be detected by measuring the transport through the quantum dot while sweeping the magnetic field at a faster speed than the coherence time.
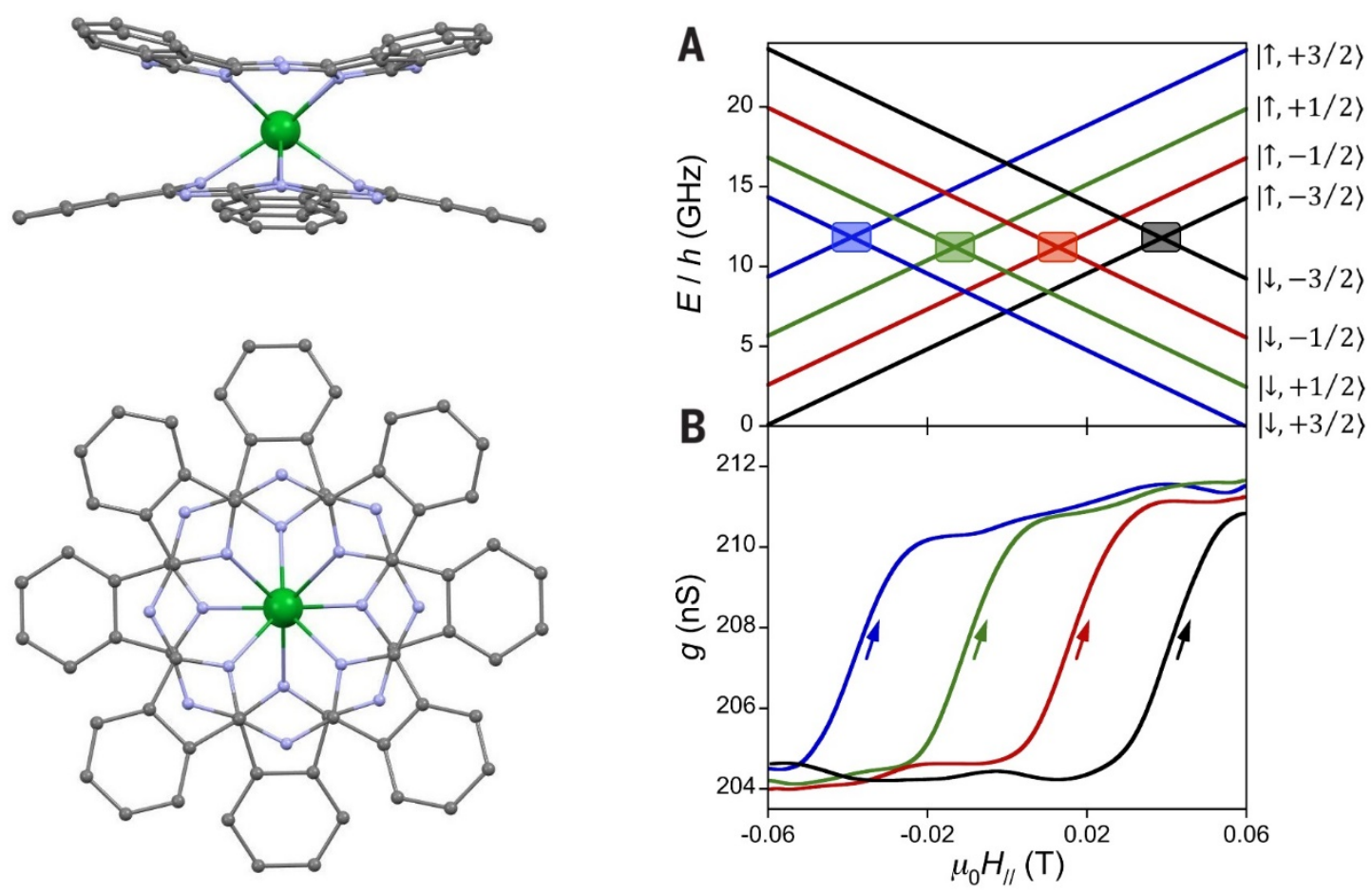
Figure 15. (Left) Representation of the molecular structure of the complex $\left[\mathrm{Tb}(\mathrm{Pc})_{2}\right]$ (side and top view). Grey, C; purple, N; green, Tb. Hydrogen atoms not shown. (Right) Zeeman diagram of the ground state $m_{\lrcorner}= \pm 6$ electronic doublet of $\mathrm{Tb}(\mathrm{III})$ in $\left[\mathrm{Tb}(\mathrm{Pc})_{2}\right]$, split by the nuclear spin $I=3 / 2$, showing the four avoided crossings (rectangles). The diagram below shows the conductivity jumps caused by the spin reversals occurring at the avoided crossings [127].

The related complex $\mathrm{Et}_{4} \mathrm{~N}\left[\mathrm{Dy}(\mathrm{Pc})_{2}\right]$ is a potential carrier of a nuclear spin-based qudit with increased dimension. Indeed most SMMs involving one or more lanthanide ions are made of Dy(III) and this metal often displays a well isolated Kramers doublet at its ground state. The inconvenient of using its nucleus to embody a qudit is that this element it is composed of several isotopes featuring two types of nuclear spin $(I=0$ and $I=5 / 2)$. The magnetic properties of each isotope were studied separately, following the preparation of isotopically enriched versions of each species [131]. Both compounds exhibit slow relaxation of the magnetization, with very similar responses to the ac susceptibility measurements. It was found however that the dependence of the relaxation time, $\tau$, with an external constant magnetic field was very different, specially, within the $\pm 20 \mathrm{mT}$ range (Fig. 16). Both isotopes exhibit zero field QTM, mirrored by low $\tau$ values than then increase with increasing the external magnetic field, as the tunneling is suppressed. The $I=5 / 2$ isotope, however, exhibits a hyperfine structure as a reflect of various additional QTM events occurring at specific magnetic field values. These processes are identified also on the hysteresis curves obtained from $\mu$-SQUID measurements on single 
crystals of this isotopologue. These events were well reproduced by fitting the data using the following Hamiltonian:

$\widehat{H}=\widehat{H}_{l f}+g_{J} \mu_{0} \mu_{B} \hat{J} \vec{H}+A_{h f} \hat{\jmath} \hat{I}+P\left(I_{z}^{2}-\frac{1}{3}(I+1) \hat{I}\right)$

In the above equation, $\widehat{H}_{l f}$ is the ligand field Hamiltonian, which is followed by the Zeeman effect term, by the hyperfine interaction component and by the contribution from the quadrupolar interaction. The simulated energy diagram, which predicts an $m_{\lrcorner}= \pm 13 / 2$ electronic ground state doublet, shows several crossing points, of which, the six most relevant ones correspond to intersections where electronic spin flips with conservation of the nuclear spin occur (ie $\left.\left|-13 / 2, \hat{I}_{z}\right\rangle \leftrightarrow\left|+13 / 2, \hat{I}_{z}\right\rangle\right)$. These six points can be exploited as the Hilbert space of a $d=6$ qudit $\left(m_{l}= \pm 5 / 2, \pm 3 / 2, \pm 1 / 2\right)$ for the realization of more complicated algorithms than is possible with the Tb analogue.
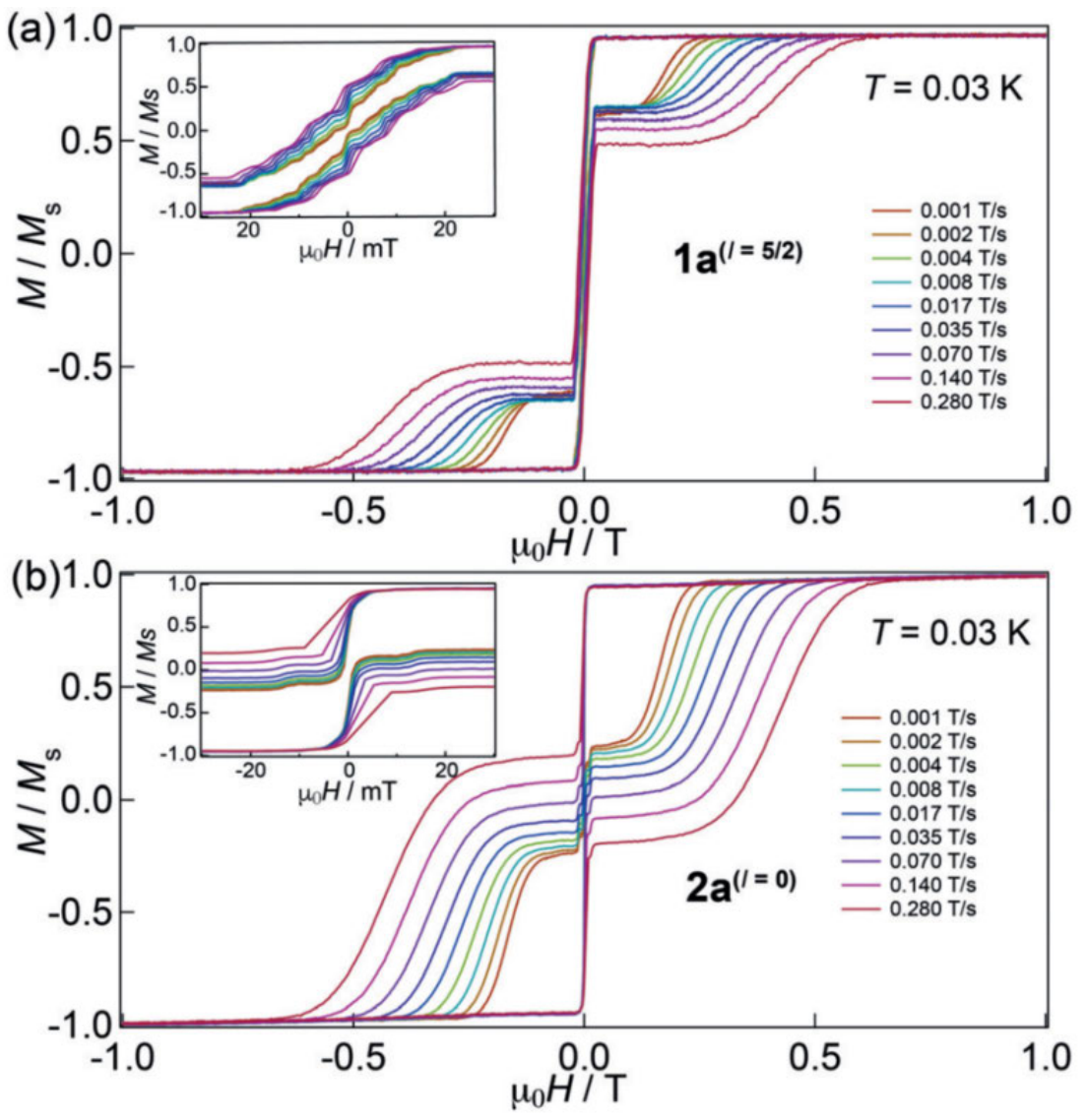
Figure 16. Magnetization as a function of the magnetic field (at $0.03 \mathrm{~K}$ ) of complex $\mathrm{Et}_{4} \mathrm{~N}\left[\mathrm{Dy}(\mathrm{Pc})_{2}\right]$ for isotopes $I=5 / 2$ (top) and $I=0$ (bottom), respectively, at various scan rates. Insets are the regions within $\pm 30 \mathrm{mT}$. Reproduced from ref. [131] with permission.

The dimension of molecular qudits based on the nuclear spin may be increased significantly by exploiting the cooperativity between RE nuclei. This was shown on the molecule $\left[\mathrm{Tb}_{2} \mathrm{Pc}^{H \times 8} \mathrm{Pc}_{2}\right] \quad\left(\mathrm{Pc}^{H \times 8}=2,3,9,10,16,17,23,24-\right.$ octahexylphthalocyaninato, Fig. 17) [132]. The electronic spins of the $\mathrm{Tb}(\mathrm{III})$ centers in this complex were found to interact ferromagnetically. This interaction provides a mechanism for coupling the Tb nuclear spins, thereby increasing the number of accessible nuclear states of the molecule and expanding the qudit dimensionality. The electronuclear states take the form $\left|\hat{J}_{z}^{a}, \hat{I}_{z}^{a}\right\rangle\left|\hat{J}_{z}^{b}, \hat{I}_{z}^{b}\right\rangle$ (a and $b$ standing for each of the $\mathrm{Tb}$ centers), of which only the projections $\left| \pm 6, \hat{I}_{z}^{a}\right\rangle\left| \pm 6, \hat{I}_{z}^{b}\right\rangle$ (ie these with $m_{J}= \pm 6$ ) are populated at low temperature. The latter amounts to an isolated Hilbert space for the nuclear spin (thus, qudit dimension $d$ ) of 16 (with $d=(2 I+1)^{n}$, where $n=2$ and $I=3 / 2$ ). Indeed, the hysteresis loops shown by this compound exhibit seven QTM events distributed symmetrically around the $H=0$ point, presumably corresponding to avoided crossings. In order to assign these transitions, a Hamiltonian including the terms for each individual $\mathrm{Tb}(\mathrm{III})$ center (Eq. 3) in addition to the coupling between both electronic spins was considered (Eq. 8).

$\widehat{H}=-2 \hat{J}_{1} \widehat{H}_{d i p} \hat{J}_{2}+\widehat{H}_{T b 1}+\widehat{H}_{T b 2}$

In this Hamiltonian, 1 and 2 refer to each Tb center, respectively. The first term is the coupling between both electronic spins, which is considered to occur 
mainly through dipolar interactions, while the other two terms include the various contributions to the energy of each $\mathrm{Tb}$ center (as in Eq. 3). Diagonalization of the Hamiltonian yields a set of parameters (eg, the hyperfine and quadrupolar parameters) allowing a simulation of the Zeeman diagram that includes the energies of all the electronuclear $\left| \pm 6, \hat{I}_{z}^{a}\right\rangle\left| \pm 6, \hat{I}_{z}^{b}\right\rangle$ eigenstates. This diagram (Fig. 17) features 100 level crossings, of which, these leading to double electronic spin flips from +6 to -6 or vice versa with conservation of the nuclear spins $\left(\left|-6, \hat{I}_{z}^{a}\right\rangle\left|-6, \hat{I}_{z}^{b}\right\rangle \leftrightarrow\left|+6, \hat{I}_{z}^{a}\right\rangle\left|+6, \hat{I}_{z}^{b}\right\rangle\right)$ exhibit high tunneling probability. They constitute avoided crossings and amount to 16 . Only seven QTM are in fact observed experimentally because some of the avoided crossings occur at the same or very close magnetic fields. The states connected by high probability tunneling at zero field are $|-6, \pm 1 / 2\rangle|-6, \pm 1 / 2\rangle$ and $|+6, \pm 1 / 2\rangle|+6, \pm 1 / 2\rangle$ in addition to the pairings $|-6, \pm 3 / 2\rangle|-6, \pm 3 / 2\rangle$ and $|+6, \pm 3 / 2\rangle|+6, \pm 3 / 2\rangle$. At $\pm 15.4 \mathrm{mT}$, the allowed spin reversal processes occur between states $\mid-6, \pm 1 /$ $2\rangle|-6, \mp 1 / 2\rangle$ and $|+6, \pm 1 / 2\rangle|+6, \mp 1 / 2\rangle$ and also among $|-6, \pm 1 / 2\rangle|-6, \mp 3 / 2\rangle$ and $|+6, \pm 1 / 2\rangle|+6, \mp 3 / 2\rangle$. At $\pm 30.4 \mathrm{mT}$, the pair of states linked by QTM are $|-6, \pm 1 / 2\rangle|-6, \pm 3 / 2\rangle$ and $|+6, \pm 1 / 2\rangle|+6, \pm 3 / 2\rangle$. The event at $\pm 45.7 \mathrm{mT}$ is ascribed to the spin flip via the states $|-6, \pm 3 / 2\rangle|-6, \mp 3 / 2\rangle$ and $\mid+6, \pm 3$ / $2\rangle|+6, \mp 3 / 2\rangle$. In this manner, the $\left[\mathrm{Tb}_{2}\right]$ complex, as a result of the interaction between its electronic spins increases the multiplicity of the nuclear spin (from four for one Tb to sixteen) which can be detected by hyperfine QTM leading to simultaneous electronic spin reversal (cotunneling). This opened the door to the increase of complexity of nuclear spin based qudits. 

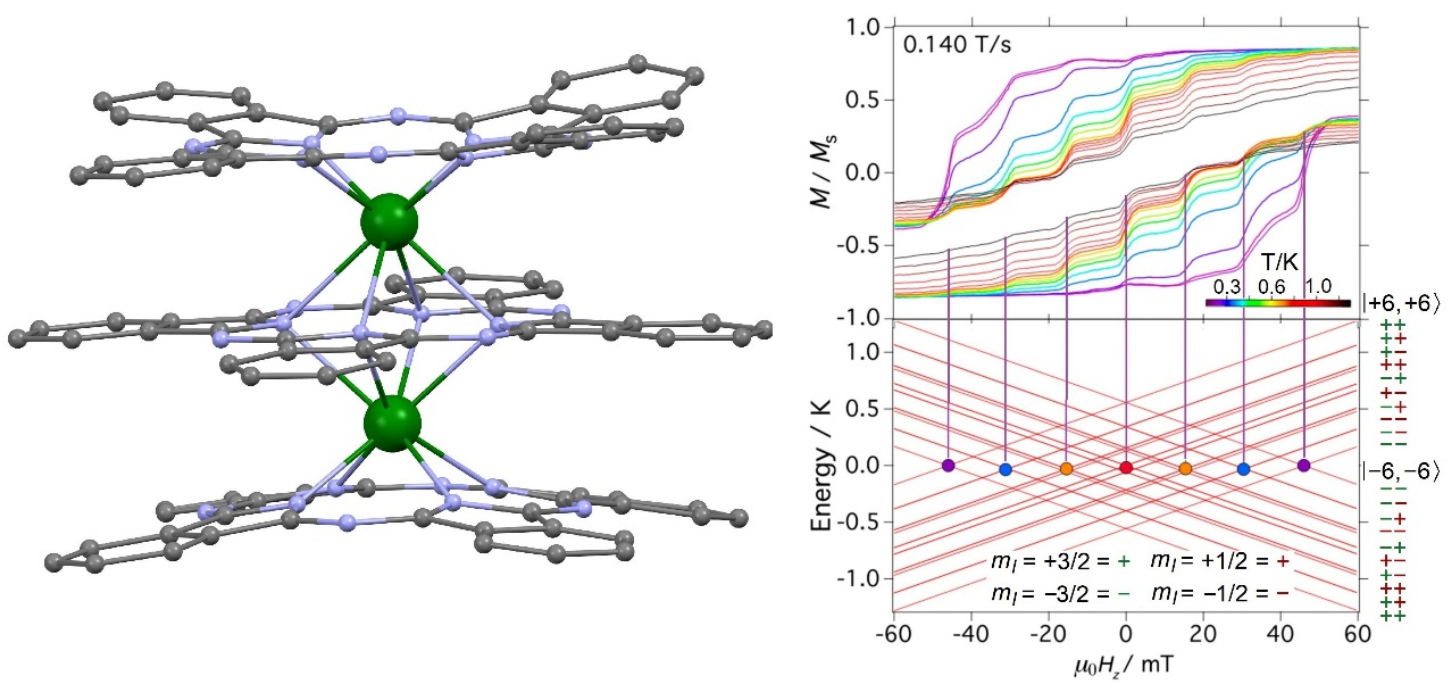

Figure 17. (Left) Representation of the molecular structure of the complex $\left[\mathrm{Tb}_{2} \mathrm{Pc}^{\mathrm{H} \times 8} \mathrm{Pc}_{2}\right]$. Grey, C; purple, N; green, Tb. Octyl groups of $\mathrm{Pc}^{H \times 8}$ ligands and hydrogen atoms not shown. (Right) A zoom of the hysteresis loops shown by $\left[\mathrm{Tb}_{2} \mathrm{Pc}^{\mathrm{H} \times 8} \mathrm{Pc}_{2}\right]$, with a staircase-like structure as a result of various QTM events. Below this diagram is Zeeman diagram as a function of $H_{z}$, of the ferromagnetic electronic ground state $| \pm 6, \pm 6\rangle$ (ie these with $m_{\lrcorner}= \pm 6$ ) of $\mathrm{Tb}(\mathrm{III})$ in this compound, split by the nuclear spins $I=3 / 2$, into the resulting electronuclear $\left| \pm 6, \hat{I}_{z}^{a}\right\rangle\left| \pm 6, \hat{I}_{z}^{b}\right\rangle$ states, indicating the positions of double spin reversal by quantum tunneling. Reproduced from ref. [132] with permission.

\subsection{Realization of Grover algorithm with the Tb nuclear spin as qudit}

Qudits may be used to implement complicated algorithms that require several qubits. For example, a qudit of dimension $d=N$ can perform the Grover algorithm, which selects a member from one unsorted data base of $N$ elements [133]. This was experimentally demonstrated by exploiting the nuclear spin states of the complex $\left[\mathrm{Tb}(\mathrm{Pc})_{2}\right](I=3 / 2, d=4$, see above $)$, as part of the threeterminal single molecule transistor described in section 5.2. Thus, after initializing the qudit, a Hadamard quantum gate was implemented. This gate 
brings a qudit to a superposition of all the states of its Hilbert space. In this case, this serves to create un unsorted quantum directory of four elements. This was carried out by means of multichromatic microwave pulse. The wave function of the qudit describing this superposition is:

$|\Psi\rangle=\frac{1}{\sqrt{N}} \sum_{n=0}^{N-1}|n\rangle$

In this function, $N$ is the dimension of the qudit (here, $d=4$ ) while $n$ represents here the label (or number) of the transition necessary to reach each state (here three possible transitions, in addition to $n=0$ for the case of the initial state) and also its wave function. After the Hadamard gate, the Grover algorithm uses a second gate on the superposition state in order to amplify the population of a researched state. This is achieved by creating a resonant condition between the researched state and the superposition, by means of a microwave pulse with a frequency that is specific to the state that one wants to select (Fig. 18). Once a particular state has been created in this manner, it can be read as described previously, measuring the transport of current through the transistor. 


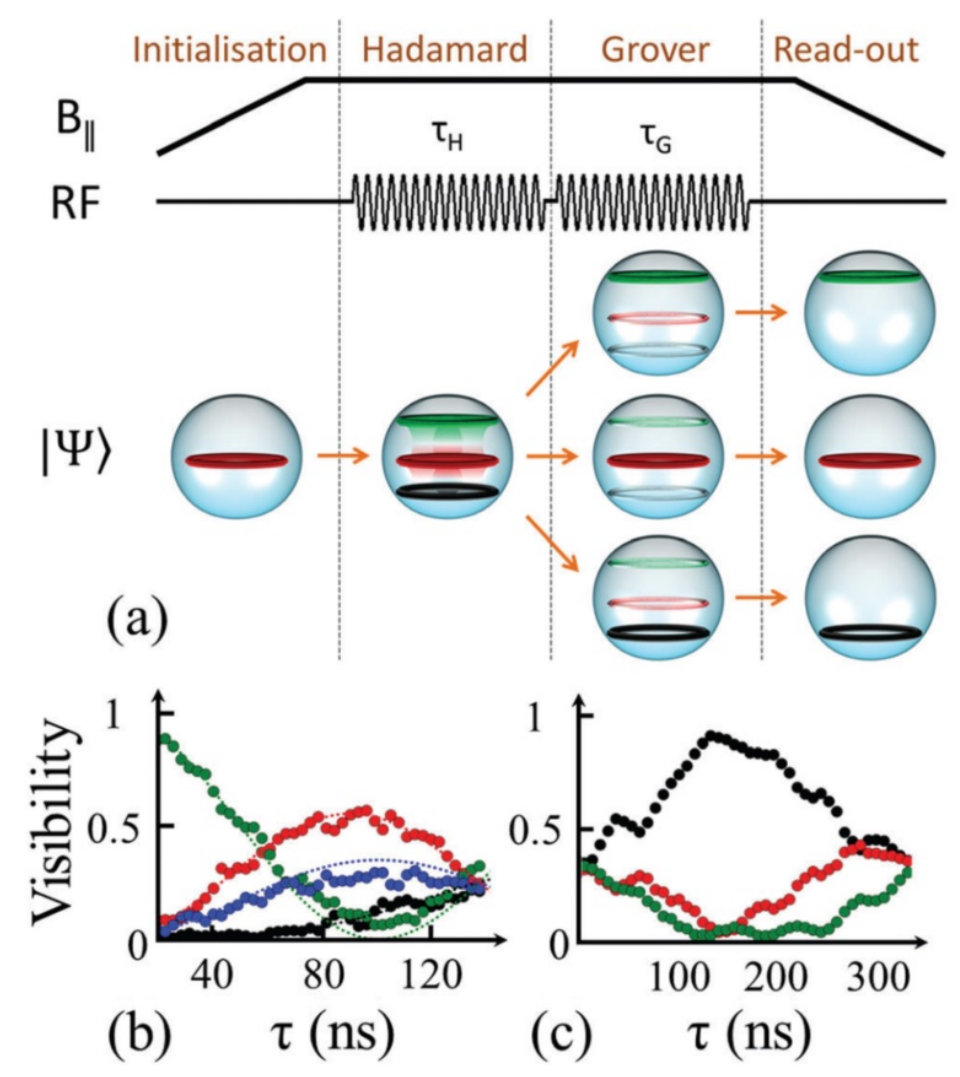

Figure 18. Sequence on the $\left[\mathrm{Tb}(\mathrm{Pc})_{2}\right]$ molecular qudit for the realization of the Grover algorithm. Following the initialization to a desired state, a Hadamard qugate is implemented to create a superposition of the four states of the qudit. From this superposotion, the researched state is amplified by implementing a pulso of a specific frequency. The final state can then be read out (see text for details). Reproduced from ref. [26] with permission.

\subsection{An error-correction protocol using the nuclear spin of $\mathrm{Yb}$ as qudit}

The complex $[\mathrm{Yb}($ trensal $)] \quad\left(\mathrm{H}_{3}\right.$ trensal $=2,2^{\prime}, 2^{\prime \prime}-$ tris(salicylideneimino)triethylamine) possesses a ${ }^{2} F_{7 / 2}$ ground state term $(L=3$, $S=1 / 2, J=7 / 2$ ) with a very well isolated $m_{J}= \pm 7 / 2$ ground doublet. The properties of the slow relaxation of its magnetization have been studied in detail [42]. Of all the isotopes of $\mathrm{Yb}$, two display a nuclear spin, I, different than zero; ${ }^{171} \mathrm{Yb}(I=1 / 2)$ and ${ }^{173} \mathrm{Yb}(I=5 / 2)$. The nuclear spin of these isotopes splits the 
energy of the electronic spin states of the ground state $m_{J}= \pm 7 / 2$ doublet, leading to a collection of $\left| \pm 7 / 2, \hat{I}_{z}\right\rangle$ electronuclear states. Single crystal pulsed EPR spectroscopy at low temperature unveils the electronic spin reversal transitions of all possible electronuclear states with $m_{J}= \pm 7 / 2$ (including that of the $I=0$ nuclei) [59]. For each isotope, these $\left|+7 / 2, \hat{I}_{z}\right\rangle \leftrightarrow\left|-7 / 2, \hat{I}_{z}\right\rangle$ transitions can be singled out by echo-detected field-swept (EDFS) X-band pulsed EPR. Standard pulsed Hahn echo sequences were employed to determine the phase memory time, $T_{m}$, of these resonances, which was found to reach a maximum, nearly constant value around $0.5 \mu$ s at low temperatures, with independence of the isotope. The fitting of inversion recovery traces of these signals was employed to determine their spin-lattice relaxation times, $T_{1}$, which from $3 \mathrm{~K}$, decays upon increase of the temperature from nearly $10 \mathrm{~ns}$ at an approximate rate of $T_{1} \propto T^{-6}$. This behavior is also independent of the isotope. Nutation experiments served to demonstrate the possibility of coherently manipulating the electronuclear states of all the isotopes. Rabi oscillations were described in detail for all of them, with varying damping rates, depending on the isotope. Later, the coherent manipulation of the nuclear magnetic states of the $d=6$ qudit contained in the ${ }^{173} \mathrm{Yb}(I=5 / 2)$ isotope of $[\mathrm{Yb}$ (trensal)], was demonstrated using NMR [27]. The system formed by the $I=5 / 2$ nucleus coupled to an effective $S=1 / 2$ spin (here formed by the $m_{J}= \pm 7 / 2$ doublet) was described by the following Hamiltonian:

$\widehat{H}=A_{\|} \hat{S}_{z} \hat{I}_{z}+A_{\perp}\left(\hat{S}_{x} \hat{I}_{x}+\hat{S}_{y} \hat{I}_{y}\right)+p \hat{I}_{z}^{2}+g \mu_{B} \hat{S} \vec{H}+g_{I} \mu_{N} \hat{I} \vec{H}$

The first term in this equation describes the hyperfine interaction between the qudit and the electronic spin, while the following terms correspond to the quadrupolar interaction of the nuclear magnetic moment and the Zeeman effect 
of both the nuclear and the electronic spin, respectively. Most of the ten $\Delta m_{l}=$ \pm 1 transitions (with conservation of the electronic spin) predicted from the wave functions of this Hamiltonian were observed in the NMR spectra. Hahn echo experiments were used to investigate the phase coherence time, $T_{2}$, of these transitions. Interestingly, the coherence time was enhanced by means of Carr-Purcell-Meibum-Gill (CPMG) sequences. The latter consist in a series of refocusing pulses that cause a decoupling of the system from the environment [134]. Transient nutation experiments served to demonstrate that arbitrary coherent superposition states could be generated with this basis. The observed Rabi oscillation frequencies confirmed that the nutation times necessary for such manipulations are significantly shorter than usual, thanks to the hyperfine coupling of the nuclear magnetic moment with the electronic spin [135]. In addition, by virtue of this coupling to the electronic effective $S=1 / 2$ spin, the $d=$ 6 computational basis of the ${ }^{173} \mathrm{Yb}(I=5 / 2)$ nucleus, was employed to encode a logical qubit protected against amplitude or phase shift errors. The electronic spin was here used as a fast ancilla to detect these amplitude shift errors, which result into unwanted $\Delta m_{l}= \pm 1$ transitions (Fig. 19). Here, the electro-nuclear states are termed using the nomenclature $\left|m_{S}, m_{I}\right\rangle, S$ being the effective spin $1 / 2\left(m_{S}= \pm 1 / 2\right)$ and $/$ being the nuclear spin $5 / 2\left(m_{l}= \pm 5 / 2, \pm 3 / 2\right.$ or $\left.\pm 1 / 2\right)$. If the states $|0\rangle$ and $|1\rangle$ of a logical qubit are encoded with this system by the two functions $|-1 / 2,-3 / 2\rangle$ and $|-1 / 2,+3 / 2\rangle$, respectively, an error consisting on the $\Delta m_{l}=+1$ amplitude shift to any superposition $|\Phi\rangle=\alpha|0\rangle+\beta|1\rangle$ of this qubit would induce the transition $|\Phi\rangle \rightarrow\left|\Phi^{+}\right\rangle$(with $\left|\Phi^{+}\right\rangle=\alpha|-1 / 2,-1 / 2\rangle+\beta \mid-1 /$ $2,+5 / 2\rangle)$, where $|\Phi\rangle$ and $\left|\Phi^{+}\right\rangle$do not overlap. To detect the error, two simultaneous MW $\pi$ pulses are applied, tuned to the transitions $|-1 / 2,-1 / 2\rangle \rightarrow$ 
$|+1 / 2,-1 / 2\rangle$ and $|-1 / 2,+5 / 2\rangle \rightarrow|+1 / 2,+5 / 2\rangle$, respectively. These pulses will rotate the electronic spin of the system only if it is in the error state $\left|\Phi^{+}\right\rangle$. The occurrence or not of a spin rotation after these two pulses is detectable, thus allowing to single out the error without collapsing the nuclear wave function. If the error has occurred, reverting the MW $\pi$ pulses brings the ancilla back to the $m_{S}=-1 / 2$, at which point, $\left|\Phi^{+}\right\rangle$may be brought back to the correct state $|\Phi\rangle$ using radiofrequency pulses. The same procedure would be used to detect and correct $\Delta m_{l}=-1$ errors, which would bring any superposition of the qubit to a state $\left|\Phi_{-}\right\rangle=\alpha|-1 / 2,-5 / 2\rangle+\beta|-1 / 2,+1 / 2\rangle$. Indeed, it follows from the above that $d=6$ is the minimal dimension needed for the qudit to implement this error correction code.

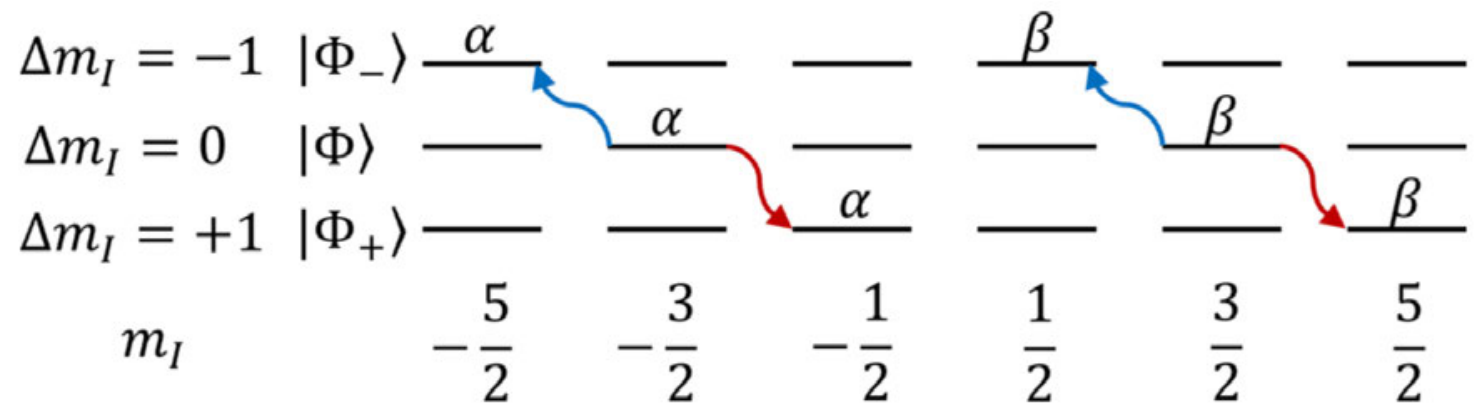

Figure 19. Representation of the transitions from the qubit encoded on the $\left|m_{S}, \pm 3 / 2\right\rangle$ states, corresponding to $\Delta m_{l}= \pm 1$ amplitude shifts, which can then be detected through the electronic effective $S=1 / 2$ ancilla, and eventually corrected (see text for details). Reproduced from ref. [27] with permission.

\section{Conclusions}

In this review we have shown the fast progress made by chemists and physicist in the race for turning molecules containing one or a few lanthanide ions into useful hardware (the qubits, qudits and qugates) for the future quantum 
processing of information. We have proposed that this process has benefited initially from the strong wave of research that emerged following the discovery of SMMs. Ten years after the advent of SMM research, lanthanide complexes were recognized as very good candidates to become slowly relaxing molecular nanomagnets and have become in fact the best performing SMMs. This collaborative strong and longstanding activity has served in part to rationalize in detail the mechanisms of the spin-lattice relaxation within lanthanide complexes, which is intimately linked to the quantum coherence of their magnetic quantum states. Understanding the mechanisms causing decoherence of these quantum states has been a main objective for most of the researchers investigating lanthanide ions as possible spin-based qubits. In recent years these studies have increasingly often coexisted with reports on the implementation of multiqubit quantum gates, resulting from successful enterprises of chemical design. The characterization of the quantum properties of the nuclear spin such as long coherence and its connection with the electronic spin has opened the way for its possible use as qudits for the coherent manipulation of its states and its use for the realization of complex multiqubit quantum gates or quantum correction protocols. The integration of lanthanide phthalocyanine single molecules with nanodevices have led to the experimental read-out and write-up of individual nuclear states, their coherent manipulation and the use of these molecules for the realization of the Grover algorithm with a list of four unsorted states. The complexity and capacity of molecular qudits and qugates is already increasing and will continue to do so. The ingredients to manufacture molecular spin-based quantum processor seem to be coming into place. However, the implementation of this molecule-based 
technology for the realization of quantum computing still needs to overcome huge challenges. The problem of connecting molecules of one qubit or more for the mutual transfer of quantum information remains an unresolved challenge. This enterprise will necessitate to incorporate additional quantum technologies such as these inherent to quantum electrodynamics [136]. Some proposals have been put forward for the incorporation of molecular qubits and qugates into superconducting planar resonators and to superconducting transmission lines [91]. In such type of devices, individual operations on a large number of qubits would be feasible as well as the control of the connections between them [137]. Such hybrid processors would allow to realize universal quantum computations on a high density of quantum information.

\section{Acknowledgements}

The authors thank financial support from Spanish Government through projects MAT2017-86826-R (OR) and PGC2018-098630-B-I00 (GA), QUANTERA for project SUMO (through Spanish PCl2018-093106), the Aragón government (DGA) through a consolidated group PLATON E31_17R (OR) and the Generalitat de Catalunya for an ICREA-Academia 2018 Prize (GA).

\section{List of symbols and acronyms}

$B_{\text {eff, effective magnetic field }}$

$\Omega_{\mathrm{R}} ;$ Rabi frequency

$\mathrm{P}_{\mathrm{Iz}}$; Landau-Zener probability

$S_{\text {eff }}$; effective spin

$\mathrm{T}_{1}$; spin-lattice relaxation time

$\mathrm{T}_{2}$; spin-spin relaxation time

${ }^{t} \mathrm{Bu}$; tert-butyl 
$\mathrm{T}_{\text {dir; }}$; direct process relaxation rate

Torbach; Orbach process relaxation rate

$\mathrm{T}_{\text {Raman; }}$ Raman process relaxation rate

$T_{Q T M ;}$ quantum tunnelling process relaxation rate

$\mathrm{T}_{\mathrm{m}}$, phase memory time

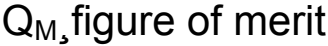

$U_{\text {eff; }}$ effective energy relaxation barrier

Ac; actinide

CASSCF-SO; complete active space self consistent field - spin orpin

Cp; cyclopentadienyl

$\mathrm{Cp}^{*}$, pentamethyl cyclopentadienyl

CCNOT, controlled-controlled-NOT

CNOT; controlled-NOT

COT; cyclooctatetraene

CPMG; Carr-Purcell-Meibum-Gill

CW-EPR, continuous wave electron paramagnetic resonance

DEER; double electron-electron resonance

EDFS EPR; echo-detected field-swept electron paramagnetic resonance

EPR; electron paramagnetic resonance

ESEEM; electron spin echo envelope modulation

HOPG; highly oriented pyrolytic graphite

HYSCORE; Hyperfine Sub-level Correlation

LBT; lanthanide-binding peptide sequence tag

Ln; lanthanide 
MALDI-TOF; Matrix Assisted Laser Desorption Ionization-Time of Flight

MW; microwave

NV center; nitrogen-vacancy center

OT,

Pc; phthalocyanine

POM; polyoxometalate

py; pyridine

QTM; quantum tunneling of the magnetization

Q-band,

Qubit; quantum bit

Qudit; quantum dit

Qugate; quantum gate

$\mathrm{RE}$; rare earth

SCXRD; single crystal X-ray diffraction

SMM; single molecule magnet

SQUID; superconducting quantum interferometer device

TREN,

trensal,

XAS; X-ray absorption spectroscopy

XMCD; X-ray magnetic circular dichroism

X-band, $9.1 \mathrm{GHz}$ frequency band

\section{References}

[1] I. M. Georgescu, S. Ashhab, F. Nori, Quantum simulation, Rev. Mod. Phys. 86, (2014), 153-185.

[2] N. Lütkenhaus, A. J. Shields, Focus on Quantum Cryptography: Theory and Practice, New J. Phys. 11, (2009), 045005. 
[3] M. A. Nielsen, I. L. Chuang, Quantum Computation and Quantum Information, Cambridge University Press, 2000.

[4] D. P. DiVincenzo, The Physical Implementation of Quantum Computation, Fortschritte der Physik 48, (2000), 771-783.

[5] T. D. Ladd, F. Jelezko, R. Laflamme, Y. Nakamura, C. Monroe, J. L. O'Brien, Quantum computers, Nature 464, (2010), 45-53.

[6] M. H. Devoret, R. J. Schoelkopf, Superconducting Circuits for Quantum Information: An Outlook, Science 339, (2013), 1169-1174.

[7] C. Monroe, J. Kim, Scaling the Ion Trap Quantum Processor, Science 339, (2013), 1164-1169.

[8] T. H. Taminiau, J. Cramer, T. van der Sar, V. V. Dobrovitski, R. Hanson, Universal control and error correction in multi-qubit spin registers in diamond, Nat. Nanotechnol. 9, (2014), 171-176.

[9] D. D. Awschalom, L. C. Bassett, A. S. Dzurak, E. L. Hu, J. R. Petta, Quantum Spintronics: Engineering and Manipulating Atom-Like Spins in Semiconductors, Science 339, (2013), 1174-1179.

[10] C. Kloeffel, D. Loss, Prospects for Spin-Based Quantum Computing in Quantum Dots, Annual Review of Condensed Matter Physics 4, (2013), 51-81.

[11] A. Ardavan, O. Rival, J. J. L. Morton, S. J. Blundell, A. M. Tyryshkin, G. A. Timco, R. E. P. Winpenny, Will spin-relaxation times in molecular magnets permit quantum information processing?, Phys. Rev. Lett. 98, (2007), 057201.

[12] M. Warner, S. Din, I. S. Tupitsyn, G. W. Morley, A. M. Stoneham, J. A. Gardener, Z. L. Wu, A. J. Fisher, S. Heutz, C. W. M. Kay, G. Aeppli, Potential for spin-based information processing in a thin-film molecular semiconductor, Nature 503, (2013), 504-508.

[13] M. A. Schlosshauer, Decoherence and the Quantum-to-Classical Transition, , , Springer, Berlin, 2008.

[14] R. Hanson, V. V. Dobrovitski, A. E. Feiguin, O. Gywat, D. D. Awschalom, Coherent dynamics of a single spin interacting with an adjustable spin bath, Science 320, (2008), 352-355.

[15] J. J. Pla, K. Y. Tan, J. P. Dehollain, W. H. Lim, J. J. L. Morton, D. N. Jamieson, A. S. Dzurak, A. Morello, A single-atom electron spin qubit in silicon, Nature 489, (2012), 541-545.

[16] J. Ferrando-Soria, E. Moreno Pineda, A. Chiesa, A. Fernandez, S. A. Magee, S. Carretta, P. Santini, I. J. Vitorica-Yrezabal, F. Tuna, G. A. Timco, E. J. L. Mclnnes, R. E. P. Winpenny, A modular design of molecular qubits to implement universal quantum gates, Nature Commun. 7, (2016), 11377.

[17] G. Aromí, D. Aguilà, P. Gamez, F. Luis, O. Roubeau, Design of magnetic coordination complexes for quantum computing, Chem. Soc. Rev. 41, (2012), 537-546.

[18] M. Atzori, L. Tesi, E. Morra, M. Chiesa, L. Sorace, R. Sessoli, RoomTemperature Quantum Coherence and Rabi Oscillations in Vanadyl Phthalocyanine: Toward Multifunctional Molecular Spin Qubits, J. Am. Chem. Soc. 138, (2016), 2154-2157.

[19] K. Sato, S. Nakazawa, R. Rahimi, T. Ise, S. Nishida, T. Yoshino, N. Mori, K. Toyota, D. Shiomi, Y. Yakiyama, Y. Morita, M. Kitagawa, K. Nakasuji, M. Nakahara, H. Hara, P. Carl, P. Hofer, T. Takui, Molecular electron- 
spin quantum computers and quantum information processing: pulsebased electron magnetic resonance spin technology applied to matter spin-qubits, J. Mater. Chem. 19, (2009), 3739-3754.

[20] S. Yamamoto, S. Nakazawa, K. Sugisaki, K. Sato, K. Toyota, D. Shiomi, T. Takui, Adiabatic quantum computing with spin qubits hosted by molecules, Phys. Chem. Chem. Phys. 17, (2015), 2742-2749.

[21] R. E. Winpenny, Quantum information processing using molecular nanomagnets as qubits, Angew. Chem. Int. Ed. 47, (2008), 7992-7994.

[22] K. Bader, D. Dengler, S. Lenz, B. Endeward, S.-D. Jiang, P. Neugebauer, J. Van Slageren, Room temperature quantum coherence in a potential molecular qubit, Nature Commun. 5, (2014), 5304.

[23] J. M. Zadrozny, J. Niklas, O. G. Poluektov, D. E. Freedman, Millisecond Coherence Time in a Tunable Molecular Electronic Spin Qubit, ACS Cent. Sci. 1, (2015), 488-492.

[24] G. Aromí, F. Luis, O. Roubeau, in Lanthanides and Actinides in Molecular Magnetism (Eds.: R. A. Layfield, M. Murugesu), Wiley-WCH, 2015, pp. 185-221.

[25] M. D. Jenkins, Y. Duan, B. Diosdado, J. J. Garcia-Ripoll, A. Gaita-Arino, C. Gimenez-Saiz, P. J. Alonso, E. Coronado, F. Luis, Coherent manipulation of three-qubit states in a molecular single-ion magnet, Phys. Rev. B 95, (2017), 8.

[26] E. Moreno-Pineda, C. Godfrin, F. Balestro, W. Wernsdorfer, M. Ruben, Molecular spin qudits for quantum algorithms, Chem. Soc. Rev. 47, (2018), 501-513.

[27] R. Hussain, G. Allodi, A. Chiesa, E. Garlatti, D. Mitcov, A. Konstantatos, K. S. Pedersen, R. De Renzi, S. Piligkos, S. Carretta, Coherent Manipulation of a Molecular Ln-Based Nuclear Qudit Coupled to an Electron Qubit, J. Am. Chem. Soc. 140, (2018), 9814-9818.

[28] C. Godfrin, A. Ferhat, R. Ballou, S. Klyatskaya, M. Ruben, W. Wernsdorfer, F. Balestro, Operating Quantum States in Single Magnetic Molecules: Implementation of Grover's Quantum Algorithm, Phys. Rev. Lett. 119, (2017), 187702.

[29] A. Abragam, B. Bleaney, Electron paramagnetic resonance of transition ions, Oxford university press, London, 1970.

[30] S. A. Al'tshuler, B. M. Kozyrev, Electron paramagnetic resonance in compounds of transition elements, Wiley, 1974.

[31] E. M. Stephens, in Lanthanide Probes in Life, Chemical and Earth Sciences: Theory and Practice (Eds.: J.-C. G. Bünzli, G. R. Chopin), Elsevier, Amsterdam, 1989.

[32] N. Ishikawa, M. Sugita, T. Ishikawa, S.-y. Koshihara, Y. Kaizu, Lanthanide Double-Decker Complexes Functioning as Magnets at the Single-Molecular Level, J. Am. Chem. Soc. 125, (2003), 8694-8695.

[33] D. N. Woodruff, R. E. P. Winpenny, R. A. Layfield, Lanthanide SingleMolecule Magnets, Chem. Rev. 113, (2013), 5110-5148.

[34] F. Pointillart, O. Cador, B. Le Guennic, L. Ouahab, Uncommon lanthanide ions in purely $4 \mathrm{f}$ Single Molecule Magnets, Coord. Chem. Rev. 346, (2017), 150-175.

[35] S. G. McAdams, A. M. Ariciu, A. K. Kostopoulos, J. P. S. Walsh, F. Tuna, Molecular single-ion magnets based on lanthanides and actinides: 
Design considerations and new advances in the context of quantum technologies, Coord. Chem. Rev. 346, (2017), 216-239.

[36] E. Bartolomé, A. Arauzo, J. Luzón, J. Bartolomé, F. Bartolomé, in Handbook of Magnetic Materials, Vol. 26 (Ed.: E. Brück), Elsevier, 2017, pp. 1-289.

[37] A. Dey, P. Kalita, V. Chandrasekhar, Lanthanide(III)-Based Single-lon Magnets, ACS Omega 3, (2018), 9462-9475.

[38] I. Bertini, G. Martini, C. Luchinat, in Handbook of Electron Spin Resonance (Eds.: C. P. Poole, H. A. Farach), American Institute of Physics, New York,, 1994, pp. 51-77.

[39] S. S. Eaton, G. R. Eaton, in Biological Magnetic Resonance, Vol. 19 (Ed.: L. J. Berliner), Kluwer Academic / Plenum Publishers, New York, 2000.

[40] K. J. Standley, R. A. Vaughan, in Electron Spin Relaxation Phenomena in Solids (Eds.: K. J. Standley, R. A. Vaughan), Springer US, Boston, MA, 1969, pp. 62-74.

[41] K. R. Meihaus, J. R. Long, Actinide-based single-molecule magnets, Dalton Trans. 44, (2015), 2517-2528.

[42] K. S. Pedersen, J. Dreiser, H. Weihe, R. Sibille, H. V. Johannesen, M. A. Sørensen, B. E. Nielsen, M. Sigrist, H. Mutka, S. Rols, J. Bendix, S. Piligkos, Design of Single-Molecule Magnets: Insufficiency of the Anisotropy Barrier as the Sole Criterion, Inorg. Chem. 54, (2015), 76007606.

[43] M. J. Giansiracusa, A. K. Kostopoulos, D. Collison, R. E. P. Winpenny, N. F. Chilton, Correlating blocking temperatures with relaxation mechanisms in monometallic single-molecule magnets with high energy barriers (Ueff $>600 \mathrm{~K}$ ), Chem. Commun., (2019).

[44] C. A. P. Goodwin, F. Ortu, D. Reta, N. F. Chilton, D. P. Mills, Molecular magnetic hysteresis at 60 kelvin in dysprosocenium, Nature 548, (2017), 439.

[45] N. Ishikawa, M. Sugita, T. Okubo, N. Tanaka, T. Lino, Y. Kaizu, Determination of ligand-field parameters and f-electronic structures of double-decker bis(phthalocyaninato)lanthanide complexes, Inorg. Chem. 42, (2003), 2440-2446.

[46] C. R. Ganivet, B. Ballesteros, G. de la Torre, J. M. Clemente-Juan, E. Coronado, T. Torres, Influence of Peripheral Substitution on the Magnetic Behavior of Single-lon Magnets Based on Homo- and Heteroleptic TbIII Bis(phthalocyaninate), Chem., Eur. J. 19, (2013), 14571465.

[47] S.-D. Jiang, B.-W. Wang, H.-L. Sun, Z.-M. Wang, S. Gao, An Organometallic Single-lon Magnet, J. Am. Chem. Soc. 133, (2011), 4730-4733.

[48] L. Ungur, J. J. Le Roy, I. Korobkov, M. Murugesu, L. F. Chibotaru, Finetuning the Local Symmetry to Attain Record Blocking Temperature and Magnetic Remanence in a Single-lon Magnet, Angew. Chem., Int. Ed. 53, (2014), 4413-4417.

[49] M. Gregson, N. F. Chilton, A. M. Ariciu, F. Tuna, I. F. Crowe, W. Lewis, A. J. Blake, D. Collison, E. J. L. Mclnnes, R. E. P. Winpenny, S. T. Liddle, A monometallic lanthanide bis(methanediide) single molecule 
magnet with a large energy barrier and complex spin relaxation behaviour, Chem. Sci. 7, (2016), 155-165.

[50] Y. S. Ding, N. F. Chilton, R. E. P. Winpenny, Y. Z. Zheng, On Approaching the Limit of Molecular Magnetic Anisotropy: A Near-Perfect Pentagonal Bipyramidal Dysprosium(III) Single-Molecule Magnet, Angew. Chem., Int. Ed. 55, (2016), 16071-16074.

[51] J. J. Le Roy, J. Cremers, I. A. Thomlinson, M. Slota, W. K. Myers, P. H. Horton, S. J. Coles, H. L. Anderson, L. Bogani, Tailored homo- and hetero- lanthanide porphyrin dimers: a synthetic strategy for integrating multiple spintronic functionalities into a single molecule, Chem. Sci. 9, (2018), 8474-8481.

[52] Z. Hu, B.-W. Dong, Z. Liu, J.-J. Liu, J. Su, C. Yu, J. Xiong, D.-E. Shi, Y. Wang, B.-W. Wang, A. Ardavan, Z. Shi, S.-D. Jiang, S. Gao, Endohedral Metallofullerene as Molecular High Spin Qubit: Diverse Rabi Cycles in Gd2@C79N, J. Am. Chem. Soc. 140, (2018), 1123-1130.

[53] S. Bertaina, S. Gambarelli, A. Tkachuk, I. N. Kurkin, B. Malkin, A. Stepanov, B. Barbara, Rare-earth solid-state qubits, Nat. Nanotechnol. 2, (2007), 39.

[54] S. Bertaina, J. H. Shim, S. Gambarelli, B. Z. Malkin, B. Barbara, SpinOrbit Qubits of Rare-Earth-Metal Ions in Axially Symmetric Crystal Fields, Phys. Rev. Lett. 103, (2009), 4.

[55] M. A. AIDamen, J. M. Clemente-Juan, E. Coronado, C. Marti-Gastaldo, A. Gaita-Arino, Mononuclear lanthanide single-molecule magnets based on polyoxometalates, J. Am. Chem. Soc. 130, (2008), 8874-+.

[56] M. J. Martinez-Perez, S. Cardona-Serra, C. Schlegel, F. Moro, P. J. Alonso, H. Prima-Garcia, J. M. Clemente-Juan, M. Evangelisti, A. GaitaArino, J. Sese, J. van Slageren, E. Coronado, F. Luis, Gd-Based SingleIon Magnets with Tunable Magnetic Anisotropy: Molecular Design of Spin Qubits, Phys. Rev. Lett. 108, (2012), 247213.

[57] M. Shiddiq, D. Komijani, Y. Duan, A. Gaita-Arino, E. Coronado, S. Hill, Enhancing coherence in molecular spin qubits via atomic clock transitions, Nature 531, (2016), 348-+.

[58] M. A. AIDamen, S. Cardona-Serra, J. M. Clemente-Juan, E. Coronado, A. Gaita-Arino, C. Marti-Gastaldo, F. Luis, O. Montero, Mononuclear Lanthanide Single Molecule Magnets Based on the Polyoxometalates $\operatorname{Ln}(\mathrm{W} 5018)(2)(9-)$ and Ln(beta(2)-SiW11O39)(2) (13-) $(\operatorname{Ln}(\mathrm{III})=\mathrm{Tb}$, Dy, Ho, Er, Tm, and Yb), Inorg. Chem. 48, (2009), 3467-3479.

[59] K. S. Pedersen, A.-M. Ariciu, S. McAdams, H. Weihe, J. Bendix, F. Tuna, S. Piligkos, Toward Molecular 4f Single-lon Magnet Qubits, J. Am. Chem. Soc. 138, (2016), 5801-5804.

[60] J. D. Rinehart, J. R. Long, Exploiting single-ion anisotropy in the design of f-element single-molecule magnets, Chem. Sci. 2, (2011), 2078-2085.

[61] V. S. Mironov, Y. G. Galyametdinov, A. Ceulemans, C. Gorller-Walrand, K. Binnemans, Room-temperature magnetic anisotropy of lanthanide complexes: A model study for various coordination polyhedra, J. Chem. Phys. 116, (2002), 4673-4685.

[62] L. Ungur, L. F. Chibotaru, in Lanthanides and Actinides in Molecular Magnetism (Eds.: M. Murugesu, R. A. Layfield), Wiley-VCH, Singapore, 2015. 
[63] L. Ungur, L. F. Chibotaru, Magnetic anisotropy in the excited states of low symmetry lanthanide complexes, Phys. Chem. Chem. Phys. 13, (2011), 20086-20090.

[64] N. F. Chilton, Design Criteria for High-Temperature Single-Molecule Magnets, Inorg. Chem. 54, (2015), 2097-2099.

[65] M. Perfetti, J. Bendix, The Multiple Faces, and Phases, of Magnetic Anisotropy, Inorg. Chem., (2019).

[66] S.-D. Jiang, S.-X. Qin, Prediction of the quantized axis of rare-earth ions: the electrostatic model with displaced point charges, Inorg. Chem. Front. 2, (2015), 613-619.

[67] F. Luis, A. Repolles, M. J. Martinez-Perez, D. Aguila, O. Roubeau, D. Zueco, P. J. Alonso, M. Evangelisti, A. Camon, J. Sese, L. A. Barrios, G. Aromi, Molecular Prototypes for Spin-Based CNOT and SWAP Quantum Gates, Phys. Rev. Lett. 107, (2011).

[68] D. Aguilà, L. A. Barrios, V. Velasco, O. Roubeau, A. Repollés, P. J. Alonso, J. Sesé, S. J. Teat, F. Luis, G. Aromí, Heterodimetallic [LnLn'] Lanthanide Complexes: Toward a Chemical Design of Two-Qubit Molecular Spin Quantum Gates, J. Am. Chem. Soc. 136, (2014), 1421514222.

[69] L. E. Rosaleny, S. Cardona-Serra, L. Escalera-Moreno, J. J. Baldovi, V. Golebiewska, K. Wlazlo, P. Casino, H. Prima-Garcia, A. Gaita-Arino, E. Coronado, Peptides as Versatile Platforms for Quantum Computing, Journal of Physical Chemistry Letters 9, (2018), 4522-4526.

[70] R. J. Blagg, L. Ungur, F. Tuna, J. Speak, P. Comar, D. Collison, W. Wernsdorfer, E. J. L. Mclnnes, L. F. Chibotaru, R. E. P. Winpenny, Magnetic relaxation pathways in lanthanide single-molecule magnets, Nat Chem 5, (2013), 673-678.

[71] N. Ishikawa, M. Sugita, W. Wernsdorfer, Quantum Tunneling of Magnetization in Lanthanide Single-Molecule Magnets: Bis(phthalocyaninato)terbium and Bis(phthalocyaninato)dysprosium Anions, Angew. Chem. Int. Ed. 44, (2005), 2931-2935.

[72] M. T. Pope, A. Muller, POLYOXOMETALATE CHEMISTRY - AN OLD FIELD WITH NEW DIMENSIONS IN SEVERAL DISCIPLINES, Angew. Chem., Int. Ed. 30, (1991), 34-48.

[73] M. T. Pope, in Handbook on the Physics and Chemistry of Rare Earths, Vol 38, Vol. 38 (Eds.: K. A. Gschneidner, J. C. G. Bunzli, V. K. Pecharsky), 2008, pp. 337-382.

[74] B. S. Bassil, U. Kortz, Recent Advances in Lanthanide-Containing Polyoxotungstates, Z. Anorg. Allg. Chem. 636, (2010), 2222-2231.

[75] F. Luis, M. J. Martínez-Pérez, O. Montero, E. Coronado, S. CardonaSerra, C. Martí-Gastaldo, J. M. Clemente-Juan, J. Sesé, D. Drung, T. Schurig, Spin-lattice relaxation via quantum tunneling in an \$\{text $\{$ Er $\}\}^{\wedge}\{3+\} \$$-polyoxometalate molecular magnet, Phys. Rev. B 82, (2010), 060403.

[76] J. J. Baldovi, L. E. Rosaleny, V. Ramachandran, J. Christian, N. S. Dalal, J. M. Clemente-Juan, P. Yang, U. Kortz, A. Gaita-Arino, E. Coronado, Molecular spin qubits based on lanthanide ions encapsulated in cubic polyoxopalladates: design criteria to enhance quantum coherence, Inorg. Chem. Front. 2, (2015), 893-897. 
[77] J. J. Baldovi, Y. Duan, C. Bustos, S. Cardona-Serra, P. Gouzerh, R. Villanneau, G. Gontard, J. M. Clemente-Juan, A. Gaita-Arino, C. Gimenez-Saiz, A. Proust, E. Coronado, Single ion magnets based on lanthanoid polyoxomolybdate complexes, Dalton Trans. 45, (2016), 16653-16660.

[78] A. Formanuik, A. M. Ariciu, F. Ortu, R. Beekmeyer, A. Kerridge, F. Tuna, E. J. L. Mclnnes, D. P. Mills, Actinide covalency measured by pulsed electron paramagnetic resonance spectroscopy, Nature Chem. 9, (2017), 578-583.

[79] S. T. Liddle, J. van Slageren, Improving f-element single molecule magnets, Chem. Soc. Rev. 44, (2015), 6655-6669.

[80] J. D. Rinehart, J. R. Long, Slow Magnetic Relaxation in a Trigonal Prismatic Uranium(III) Complex, J. Am. Chem. Soc. 131, (2009), 1255812559.

[81] K. R. Meihaus, S. G. Minasian, W. W. Lukens, S. A. Kozimor, D. K. Shuh, T. Tyliszczak, J. R. Long, Influence of Pyrazolate vs NHeterocyclic Carbene Ligands on the Slow Magnetic Relaxation of Homoleptic Trischelate Lanthanide(III) and Uranium(III) Complexes, J. Am. Chem. Soc. 136, (2014), 6056-6068.

[82] L. C. J. Pereira, C. Camp, J. T. Coutinho, L. Chatelain, P. Maldivi, M. Almeida, M. Mazzanti, Single-Molecule-Magnet Behavior in Mononuclear Homoleptic Tetrahedral Uranium(III) Complexes, Inorg. Chem. 53, (2014), 11809-11811.

[83] F. Moro, D. P. Mills, S. T. Liddle, J. van Slageren, The Inherent SingleMolecule Magnet Character of Trivalent Uranium, Angew. Chem. Int. Ed. 52, (2013), 3430-3433.

[84] J. J. Le Roy, S. I. Gorelsky, I. Korobkov, M. Murugesu, Slow Magnetic Relaxation in Uranium(III) and Neodymium(III) Cyclooctatetraenyl Complexes, Organometallics 34, (2015), 1415-1418.

[85] D. M. King, P. A. Cleaves, A. J. Wooles, B. M. Gardner, N. F. Chilton, F. Tuna, W. Lewis, E. J. L. Mclnnes, S. T. Liddle, Molecular and electronic structure of terminal and alkali metal-capped uranium(V) nitride complexes, Nature Commun. 7, (2016), 13773.

[86] D. M. King, F. Tuna, J. McMaster, W. Lewis, A. J. Blake, E. J. L. Mclnnes, S. T. Liddle, Single-Molecule Magnetism in a Single-lon Triamidoamine Uranium(V) Terminal Mono-Oxo Complex, Angew. Chem. Int. Ed. 52, (2013), 4921-4924.

[87] J. Flores Gonzalez, F. Pointillart, O. Cador, Hyperfine coupling and slow magnetic relaxation in isotopically enriched Dyll mononuclear singlemolecule magnets, Inorg. Chem. Front. 6, (2019), 1081-1086.

[88] F. Pointillart, K. Bernot, S. Golhen, B. Le Guennic, T. Guizouarn, L. Ouahab, O. Cador, Magnetic Memory in an Isotopically Enriched and Magnetically Isolated Mononuclear Dysprosium Complex, Angew. Chem., Int. Ed. 54, (2015), 1504-1507.

[89] E. Moreno-Pineda, M. Damjanovic, O. Fuhr, W. Wernsdorfer, M. Ruben, Nuclear Spin Isomers: Engineering a Et4N DyPc2 Spin Qudit, Angew. Chem., Int. Ed. 56, (2017), 9915-9919.

[90] W. B. Mims, K. Nassau, J. D. McGee, SPECTRAL DIFFUSION IN ELECTRON RESONANCE LINES, Physical Review 123, (1961), 2059\&. 
[91] M. D. Jenkins, D. Zueco, O. Roubeau, G. Aromí, J. Majer, F. Luis, A scalable architecture for quantum computation with molecular nanomagnets, Dalton Trans. 45, (2016), 16682-16693.

[92] S. Ghosh, S. Datta, L. Friend, S. Cardona-Serra, A. Gaita-Arino, E. Coronado, S. Hill, Multi-frequency EPR studies of a mononuclear holmium single-molecule magnet based on the polyoxometalate HoIII(W5O18)(2) (9-), Dalton Trans. 41, (2012), 13697-13704.

[93] K. N. Allen, B. Imperiali, Lanthanide-tagged proteins - an illuminating partnership, Curr. Opin. Chem. Biol. 14, (2010), 247-254.

[94] N. Alonso-Garcia, I. Garcia-Rubio, J. A. Manso, R. M. Buey, H. Urien, A. Sonnenberg, G. Jeschke, J. M. de Pereda, Combination of X-ray crystallography, SAXS and DEER to obtain the structure of the FnIII-3,4 domains of integrin alpha 6 beta 4, Acta Crystallographica Section DStructural Biology 71, (2015), 969-985.

[95] D. Barthelmes, M. Granz, K. Barthelmes, K. N. Allen, B. Imperiali, T. Prisner, H. Schwalbe, Encoded loop-lanthanide-binding tags for longrange distance measurements in proteins by NMR and EPR spectroscopy, J. Biomol. NMR 63, (2015), 275-282.

[96] M. J. Giansiracusa, E. Moreno-Pineda, R. Hussain, R. Marx, M. M. Prada, P. Neugebauer, S. Al-Badran, D. Collison, F. Tuna, J. van Slageren, S. Carretta, T. Guidi, E. J. L. McLnnes, R. E. P. Winpenny, N. F. Chilton, Measurement of Magnetic Exchange in Asymmetric Lanthanide Dimetallics: Toward a Transferable Theoretical Framework, J. Am. Chem. Soc. 140, (2018), 2504-2513.

[97] D. Aguila, L. A. Barrios, F. Luis, A. Repolles, O. Roubeau, S. J. Teat, G. Aromí, Synthesis and Properties of a Family of Unsymmetric Dinuclear Complexes of $\operatorname{Ln}(\mathrm{III})$ (Ln = Eu, Gd, Tb), Inorg. Chem. 49, (2010), 67846786.

[98] D. Aguila, L. A. Barrios, V. Velasco, L. Arnedo, N. Aliaga-Alcalde, M. Menelaou, S. J. Teat, O. Roubeau, F. Luis, G. Aromi, Lanthanide Contraction within a Series of Asymmetric Dinuclear Ln(2) Complexes, Chem., Eur. J. 19, (2013), 5881-5891.

[99] N. Dalla-Favera, J. Hamacek, M. Borkovec, D. Jeannerat, G. Ercolani, C. Piguet, Tuneable Intramolecular Intermetallic Interactions as a New Tool for Programming Linear Heterometallic $4 \mathrm{f}-4 \mathrm{f}$ Complexes, Inorg. Chem. 46, (2007), 9312-9322.

[100] N. Ishikawa, T. lino, Y. Kaizu, Interaction between f-electronic systems in dinuclear lanthanide complexes with phthalocyanines, J. Am. Chem. Soc. 124, (2002), 11440-11447.

[101] J. P. Costes, F. Dahan, F. Nicodème, Structure-based description of a step-by-step synthesis of homo- and heterodinuclear ( $\left.4 f, 4 f^{\prime}\right)$ lanthanide complexes, Inorg. Chem. 42, (2003), 6556-6563.

[102] J.-P. Costes, F. Nicodème, Unequivocal Synthetic Pathway to Heterodinuclear $\left(4 f, 4 f^{\prime}\right)$ Complexes: Magnetic Study of Relevant (LnIII, GdIII) and (GdIII, LnIII) Complexes, Chem., Eur. J. 8, (2002), 3442-3447.

[103] X.-Y. Chen, Y. Bretonnière, J. Pécaut, D. Imbert, J.-C. Bünzli, M. Mazzanti, Selective Self-Assembly of Hexameric Homo- and Heteropolymetallic Lanthanide Wheels: Synthesis, Structure, and Photophysical Studies, Inorg. Chem. 46, (2007), 625-637. 
[104] Y. H. Lan, S. Klyatskaya, M. Ruben, O. Fuhr, W. Wernsdorfer, A. Candini, V. Corradini, A. L. Rizzini, U. del Pennino, F. Troiani, L. Joly, D. Klar, H. Wende, M. Affronte, Magnetic interplay between two different lanthanides in a tris-phthalocyaninato complex: a viable synthetic route and detailed investigation in the bulk and on the surface, J. Mater. Chem. C 3, (2015), 9794-9801.

[105] S. Faulkner, S. J. A. Pope, Lanthanide-Sensitized Lanthanide Luminescence: Terbium-Sensitized Ytterbium Luminescence in a Trinuclear Complex, J. Am. Chem. Soc. 125, (2003), 10526-10527.

[106] D. J. Lewis, P. B. Glover, M. C. Solomons, Z. Pikramenou, Purely Heterometallic Lanthanide(III) Macrocycles through Controlled Assembly of Disulfide Bonds for Dual Color Emission, J. Am. Chem. Soc. 133, (2011), 1033-1043.

[107] P. Zhu, N. Pan, R. Li, J. Dou, Y. Zhang, D. Y. Y. Cheng, D. Wang, D. K. P. Ng, J. Jiang, Electron-Donating Alkoxy-Group-Driven Synthesis of Heteroleptic Tris(phthalocyaninato) Lanthanide(III) Triple-Deckers with Symmetrical Molecular Structure, Chem., Eur. J. 11, (2005), 1425-1432.

[108] R. Sato, K. Suzuki, M. Sugawa, N. Mizuno, Heterodinuclear LanthanoidContaining Polyoxometalates: Stepwise Synthesis and Single-Molecule Magnet Behavior, Chem., Eur. J. 19, (2013), 12982-12990.

[109] L. S. Natrajan, A. J. L. Villaraza, A. M. Kenwright, S. Faulkner, Controlled preparation of a heterometallic lanthanide complex containing different lanthanides in symmetrical binding pockets, Chem. Commun., (2009), 6020-6022.

[110] M. P. Placidi, A. J. L. Villaraza, L. S. Natrajan, D. Sykes, A. M. Kenwright, S. Faulkner, Synthesis and Spectroscopic Studies on AzoDye Derivatives of Polymetallic Lanthanide Complexes: Using Diazotization to Link Metal Complexes Together, J. Am. Chem. Soc. 131, (2009), 9916-9917.

[111] J. A. Tilney, T. J. Sorensen, B. P. Burton-Pye, S. Faulkner, Selfassembly between dicarboxylate ions and a binuclear europium complex: formation of stable adducts and heterometallic lanthanide complexes, Dalton Trans. 40, (2011), 12063-12066.

[112] M. Seitz, A. G. Oliver, K. N. Raymond, The Lanthanide Contraction Revisited, J. Am. Chem. Soc. 129, (2007), 11153-11160.

[113] S. Floquet, M. Borkovec, G. Bernardinelli, A. Pinto, L. A. Leuthold, G. Hopfgartner, D. Imbert, J. C. G. Bunzli, C. Piguet, Programming heteropolymetallic lanthanide helicates: Thermodynamic recognition of different metal ions along the strands, Chem., Eur. J. 10, (2004), 10911105.

[114] N. Andre, R. Scopelliti, G. Hopfgartner, C. Piguet, J. C. G. Bunzli, Discriminating between lanthanide ions: self-assembly of heterodimetallic triple-stranded helicates, Chem. Commun., (2002), 214-215.

[115] T. Riis-Johannessen, N. Dalla Favera, T. K. Todorova, S. M. Huber, L. Gagliardi, C. Piguet, Understanding, Controlling and Programming Cooperativity in Self-Assembled Polynuclear Complexes in Solution, Chem., Eur. J. 15, (2009), 12702-12718.

[116] F. Artizzu, F. Quochi, L. Marchiò, R. F. Correia, M. Saba, A. Serpe, A. Mura, M. L. Mercuri, G. Bongiovanni, P. Deplano, Cover Picture: Ln3Q9 as a Molecular Framework for Ion-Size-Driven Assembly of 
Heterolanthanide (Nd, Er, Yb) Multiple Near-Infrared Emitters (Chem. Eur. J. 10/2015), Chem., Eur. J. 21, (2015), 3833-3833.

[117] F. Guyon, A. Pondaven, J.-M. Kerbaol, M. L'Her, From the Single- to the Triple-Decker Sandwich. Effect of Stacking on the Redox and UV-Visible Spectroscopic Properties of Lutetium(III) 1,2-Naphthalocyaninate Complexes, Inorg. Chem. 37, (1998), 569-576.

[118] T. Kazuhiro, S. Jun-ichi, I. Makoto, F. Yoshio, O. Hisashi, Synthesis and Characterization of Triple-decker Sandwich Dinuclear La(III) and Lu(III) Complexes of 2,3,9,10,16,17,23,24-Octabutoxyphthalocyanine, Chem. Lett. 27, (1998), 173-174.

[119] K. Kuninobu, A. Munenori, M. Hideharu, I. Mikio, Preparation of New Phthalocyanine Complexes of Yttrium(III) and Some Lanthanoid(III) lons, Chem. Lett. 15, (1986), 1095-1098.

[120] N. Ishikawa, T. lino, Y. Kaizu, Determination of Ligand-Field Parameters and f-Electronic Structures of Hetero-Dinuclear Phthalocyanine Complexes with a Diamagnetic Yttrium(III) and a Paramagnetic Trivalent Lanthanide Ion, The Journal of Physical Chemistry A 106, (2002), 95439550.

[121] D. Aguila, V. Velasco, L. A. Barrios, J. Gonzalez-Fabra, C. Bo, S. J. Teat, O. Roubeau, G. Aromi, Selective Lanthanide Distribution within a Comprehensive Series of Heterometallic LnPr Complexes, Inorg. Chem. 57, (2018), 8429-8439.

[122] J. Gonzalez-Fabra, N. A. G. Bandeira, V. Velasco, L. A. Barrios, D. Aguila, S. J. Teat, O. Roubeau, C. Bo, G. Aromi, Thermodynamic Stability of Heterodimetallic LnLn Complexes: Synthesis and DFT Studies, Chem., Eur. J. 23, (2017), 5117-5125.

[123] S. Pirandola, S. Mancini, S. L. Braunstein, D. Vitali, Minimal qudit code for a qubit in the phase-damping channel, Phys. Rev. A 77, (2008), 032309.

[124] M. K. Singh, N. Yadav, G. Rajaraman, Record high magnetic exchange and magnetization blockade in Ln2@C79N (Ln = Gd(iii) and Dy(iii)) molecules: a theoretical perspective, Chem. Commun. 51, (2015), 17732-17735.

[125] B. E. Kane, A silicon-based nuclear spin quantum computer, Nature 393, (1998), 133-137.

[126] S. Thiele, R. Vincent, M. Holzmann, S. Klyatskaya, M. Ruben, F. Balestro, W. Wernsdorfer, Electrical Readout of Individual Nuclear Spin Trajectories in a Single-Molecule Magnet Spin Transistor, Phys. Rev. Lett. 111, (2013), 037203.

[127] S. Thiele, F. Balestro, R. Ballou, S. Klyatskaya, M. Ruben, W. Wernsdorfer, Electrically driven nuclear spin resonance in singlemolecule magnets, Science 344, (2014), 1135-1138.

[128] W. Wernsdorfer, R. Sessoli, A. Caneschi, D. Gatteschi, A. Cornia, D. Mailly, Landau-Zener method to study quantum phase interference of Fe8 molecular nanomagnets (invited), J. Appl. Phys. 87, (2000), 54815486.

[129] R. Vincent, S. Klyatskaya, M. Ruben, W. Wernsdorfer, F. Balestro, Electronic read-out of a single nuclear spin using a molecular spin transistor, Nature 488, (2012), 357. 
[130] R. Rahman, C. J. Wellard, F. R. Bradbury, M. Prada, J. H. Cole, G. Klimeck, L. C. L. Hollenberg, High Precision Quantum Control of Single Donor Spins in Silicon, Phys. Rev. Lett. 99, (2007), 036403.

[131] E. Moreno-Pineda, M. Damjanović, O. Fuhr, W. Wernsdorfer, M. Ruben, Nuclear Spin Isomers: Engineering a Et4N[DyPc2] Spin Qudit, Angew. Chem. Int. Ed. 56, (2017), 9915-9919.

[132] E. Moreno-Pineda, S. Klyatskaya, P. Du, M. Damjanović, G. Taran, W. Wernsdorfer, M. Ruben, Observation of Cooperative Electronic Quantum Tunneling: Increasing Accessible Nuclear States in a Molecular Qudit, Inorg. Chem. 57, (2018), 9873-9879.

[133] L. K. Grover, Quantum Mechanics Helps in Searching for a Needle in a Haystack, Phys. Rev. Lett. 79, (1997), 325-328.

[134] G. de Lange, Z. H. Wang, D. Ristè, V. V. Dobrovitski, R. Hanson, Universal Dynamical Decoupling of a Single Solid-State Spin from a Spin Bath, Science 330, (2010), 60-63.

[135] J. S. Hodges, J. C. Yang, C. Ramanathan, D. G. Cory, Universal control of nuclear spins via anisotropic hyperfine interactions, Phys. Rev. A 78, (2008), 010303.

[136] J. Majer, J. M. Chow, J. M. Gambetta, J. Koch, B. R. Johnson, J. A. Schreier, L. Frunzio, D. I. Schuster, A. A. Houck, A. Wallraff, A. Blais, M. H. Devoret, S. M. Girvin, R. J. Schoelkopf, Coupling superconducting qubits via a cavity bus, Nature 449, (2007), 443.

[137] A. Gaita-Ariño, F. Luis, S. Hill, E. Coronado, Molecular spins for quantum computation, Nature Chem. 11, (2019), 301-309. 